Department of Informatics

\title{
Spine as an Engine: Effect of Spine Morphology on Spine-Driven Quadruped Locomotion
}

A dissertation submitted to the Faculty of Economics, Business

Administration and Information Technology of the University of Zurich

for the degree of

Doctor of Science (Ph.D.)

by

Qian Zhao

from China

Accepted on the recommendation of

Prof. Dr. Rolf Pfeifer

Prof. Dr. Cecilia Laschi

Prof. Dr. Fumiya Iida 
The Faculty of Economics, Business Administration and Information Technology of the University of Zurich herewith permits the publication of the aforementioned dissertation without expressing any opinion on the views contained therein.

Zurich, August 16, 2014

Head of the Ph.D. program in informatics: Prof. Dr. Abraham Bernstein 


\section{Acknowledgements}

First of all, I would like to thank Prof. Dr. Rolf Pfeifer for offering me the opportunity to carry out my research at the Artificial Intelligence Laboratory. I highly appreciate his support and encouragement during my studies. Further, I would like to thank him for providing this excellent, inspiring, crazy, international research environment. Many thanks also to Prof. Dr. Fumiya Iida and Prof. Dr. Cecilia Laschi for being co-reviewers and to Prof. Dr. Elaine M. Huang for joining the Ph.D. committee.

During the expedition of my Ph.D. study, I have been extremely lucky to work together with Dr. Hidenobu Sumioka and Dr. Kohei Nakajima. I sincerely appreciate all the inspiring discussions and unreserved sharing of research experience from them. They not only trained me to grow as a researcher, but also encouraged me to be an independent thinker. Without their kind and patient instruction, my research career would not get started and this thesis would not be finished. Many thanks to my colleagues Tao Li, Vu Quy Hung, Farrukh Iqbal Sheikh, Xiaoxiang $\mathrm{Yu}$ for their thoughtful and encouraging inputs to my work and Nathan Labhart for his humor, warm smiles and support. I would like to also acknowledge all my colleagues at this AI lab for support and encouragements.

This research was mainly funded by the European Community's Seventh Framework Program FP7-ICT-248311 (AMARSi). My sincere appreciation goes to all of partners who made this project such a wonderful and open working environment.

Last but not least, I would like to thank my parents for their continuous and unconditional support. Special thanks to my husband Baozhong Zhang, who stands and shares with me both the great and the difficult moments of my life. 



\section{Abstract}

Legged robots have attracted much attention from robotic researchers due to their potential application in rough terrains. Most existing quadruped robots are very similar in their morphology and feature a single rigid body with four legs which are individually actuated by hip and/or knee joints. However, the resulting locomotion behavior is much more constrained than their natural counterparts in terms of speed, energy efficiency, maneuverability, and adaptability to rough terrain. From a biological point of view, one of the major morphological differences between legged robots and vertebrate animals is the spine. The function of the spine has been hypothesized to be a main component to drive locomotion in a biological concept called "Spinal Engine".

In this thesis, aiming to investigate the locomotor function of a spine and its morphological effect in quadruped locomotion, we design several biologically inspired spines and embed them to a series of robots in the real world and in simulation. Our work mainly focuses on the following three important questions. First, is it possible to generate spine-driven locomotion in quadruped robots? Second, if it is, then how does spine morphology affect locomotion? Last, how to coordinate the leg and the spine movements?

Based on our research objective, a series of experiments have been carried out in different robotic platforms (e.g., the Kitty robot, the simulation model, the Renny robot). First, we demonstrate how versatile behaviors (bounding gait, trotting gait, and turning behavior) can be generated exclusively by the spine movements through dynamical interaction between the controller, the body, and the environment in the Kitty robot endowed with a biologically inspired spine. Then, information theory is taken to characterize how the change of spine morphology (e.g., the position of a virtual spinal joint) induces such dynamical interaction in the bounding gait. Next, we develop quadruped models with different numbers of spinal joints in simulation to study how such a feature generates and affects locomotion. Simulation results reveal that the model with two spinal joints can greatly enhance the stability and speed of locomotion and suggest that such a model might be reasonable to emulate a biological spine model, compared to the model with one joint, such as the Kitty robot. In addition to be a mechanical component connecting the front and rear legs, the function of the spine in the Kitty robot has been examined by regarding it as a computational resource in the context of morphological computation. Due to the compliance and nonlinearity embedded in this actuated flexible spine, such a compliant spine together 
with linear and static readouts and feedback loops is able to encode movement patterns, produce rhythmic patterns, and learn new patterns. At the end, a pneumatically-driven quadruped robot called Renny with configurable spine morphology has been developed to investigate the effect of the spine stiffness on quadruped locomotion. Furthermore, the way to coordinate the leg and the spine is also investigated. Experimental results are in agreement with biological findings to a large extent, which reveals how such properties affect locomotion in nature.

This thesis sheds new light on the spine-driven quadruped locomotion and reveals the function of the spine in quadruped locomotion. 


\section{Zusammenfassung}

Laufroboter haben aufgrund ihrer potentiellen Anwendbarkeit auf unebenem Terrain viel Aufmerksamkeit bei Robotikforschern erregt. Die meisten vierbeinigen Roboter sind sich in ihrer Morphologie sehr Ähnlich und bestehen aus einem starren Körper mit vier individuell an Hüftund/oder Kniegelenken angetriebenen Beinen. Ihr daraus resultierendes Fortbewegungsverhalten ist jedoch bezüglich Geschwindigkeit, Energieeffizienz, Manövrierbarkeit und Anpassungsfähigkeit an unwegsames Gelände sehr viel eingeschränkter als das ihrer natürlichen Pendants. Aus biologischer Sicht ist eine der morphologischen Hauptunterschiede zwischen Laufrobotern und Wirbeltieren die Wirbelsäule. In dem biologischen Konzept des „Spinal Engine“ wird angenommen, dass die Wirbelsäule eine Schlüsselrolle bei der Fortbewegung spielt.

In dieser Arbeit, darauf abzielend, die Rolle der Wirbelsäule für die Fortbewegung sowie ihres morphologischen Einflusses bei vierbeiniger Fortbewegung zu untersuchen, entwerfen wir mehrere biologisch inspirierte Wirbelsäulen und integrieren sie in ein Reihe von echten und simulierten Robotern. Unsere Arbeit zielt dabei hauptsächlich auf drei wichtige Fragestellungen ab. Erstens, ist es möglich, Wirbelsäulengetriebene Fortbewegung in Robotern zu generieren? Zweitens, falls das der Fall ist, wie beeinflusst die Morphologie der Wirbelsäule die Fortbewegung? Schliesslich, wie koordiniert man die Bewegungen der Beine mit denen der Wirbelsäule?

Basierend auf diesen Forschungszielsetzungen wurde eine Reihe von Experimenten in verschiedenen Roboterplattformen durchgeführt (z.B. der Kitty Roboter, das simulierte Modell und der Renny Roboter). Zunächst führen wir im Kitty Roboter, ausgestattet mit einer biologisch inspirierten Wirbelsäule, vor, wie ausschliesslich durch Wirbelsäulenbewegungen, durch dynamische Interaktion zwischen Regler, Körper und Umwelt, vielseitige Verhalten (Sprung, Trab und Drehen) generiert werden können. Dann benutzen wir die Informationstheorie dazu, zu charakterisieren, wie die Änderung der Wirbelsäulenmorphologie (z.B. die Position eines virtuellen Wirbelsäulengelenks) eine solche dynamische Interaktion in der Sprunggangart induzieren. Weiter entwickeln wir in Simulation vierbeinige Modelle mit unterschiedlicher Anzahl an Wirbelsäulengelenken, um herauszufinden, wie sich dieses Merkmal auf die Fortbewegung auswirkt. Die Simulationen zeigen, dass das Modell mit zwei Wirbelsäulengelenken die Stabilität und Geschwindigkeit der Fortbewegung enorm steigern kann und legen nahe, dass solch ein Modell, verglichen mit dem Modell mit nur einem Gelenk, wie beim Kitty Roboter, geeignet sein könnte, 
um eine biologische Wirbelsäule zu emulieren. Die Wirbelsäule des Kitty Roboters wurde, über ihre Funktion als mechanische Verbindungskomponente zwischen Vorder- und Hinterbeinen hinaus, auf seine Funktion hin untersucht, als Rechenressource im Kontext von morphological computation zu fungieren. Dank der flexiblen Nachgiebigkeit und Nichtlinearität dieser angetriebenen Wirbelsäule, kann sie dazu benutzt werden, durch lineare und statische Auslese- und Feedbackverbindungen Bewegungsmuster zu kodieren, rhythmische Muster zu generieren und neue Muster zu lernen. Zuletzt wurde ein pneumatisch angetriebener vierbeiniger Roboter namens Renny mit konfigurierbarer Wirbelsäulenmorphologie entwickelt, um die Auswirkung der Wirbelsäulensteifheit auf die Fortbewegung zu untersuchen. Desweiteren wird die Koordinierung von Beinen und Wirbelsäule untersucht. Experimentelle Resultate stimmen weitgehend mit biologischen Befunden überein, welche solche Eigenschaften in der Natur untersuchen.

Die vorliegende Arbeit gibt neue Aufschlüsse über die Wirbelsäulengetriebene vierbeinige Fortbewegung und klärt die Funktion der Wirbelsäule in vierbeiniger Fortbewegung. 


\section{Contents}

1 Introduction 1

1.1 Motivation . . . . . . . . . . . . . . . . . . 1

1.2 Contributions of a Spine to Legged Animal Locomotion . . . . . . . . . . . 2

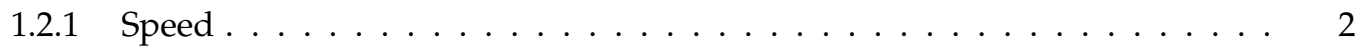

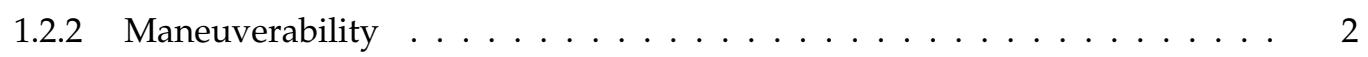

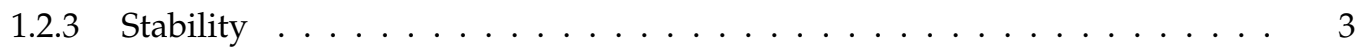

1.2.4 Energy Storage . . . . . . . . . . . . . . . . . . . 3

1.3 Early Attempts to Design Quadruped Robots . . . . . . . . . . . . . . . 3

1.3.1 Robots with a Rigid Spine . . . . . . . . . . . . . . . . . . . . 4

1.3.2 Robots Emphasizing Control on a Simple Spinal Joint . . . . . . . . . . 4

1.3.3 Robots with a Focus on Spine Morphology . . . . . . . . . . . . . . 5

1.4 Research Questions and Hypotheses . . . . . . . . . . . . . . . 7

1.5 Research Methodology . . . . . . . . . . . . . . . . . . . . . . . . . . . . . .

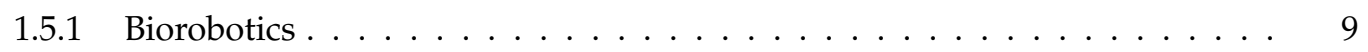

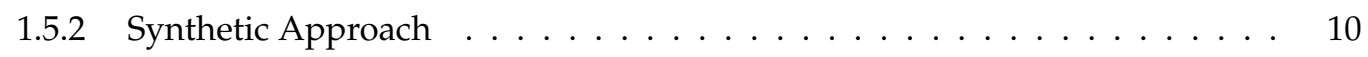

1.5 .3 Information Theory . . . . . . . . . . . . . . . . 10

1.5.4 Morphological Computation . . . . . . . . . . . . . . . 11

1.6 Issues Not Considered in this Thesis . . . . . . . . . . . . . . . . . . . 11

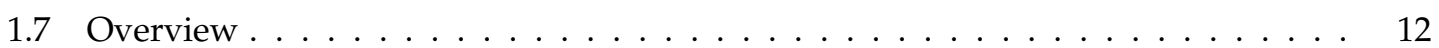

2 Spinal Engine Hypothesis and Biological Properties of a Spine 15

2.1 Hypothesis on Legged Locomotion . . . . . . . . . . . . . . . . . . . . . . 15

2.2 Spinal Engine Hypothesis … . . . . . . . . . . . . . . . . . 17

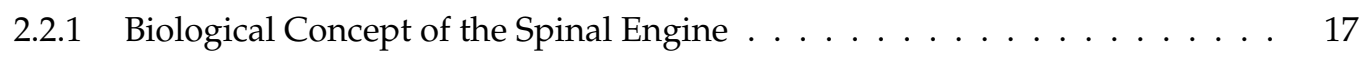

2.2 .2 Evolution of a Spine . . . . . . . . . . . . . . . . . . 17

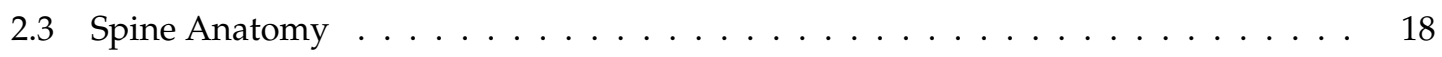

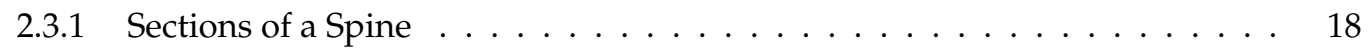

2.3.2 Components of a Spine $\ldots \ldots \ldots \ldots \ldots$ 
2.4 Spinal Movements . . . . . . . . . . . . . . . . . . . . . . 19

2.4 Type of Spinal Movements . . . . . . . . . . . . . . . . . 20

2.4 .2 Intervertebral Joint Movements . . . . . . . . . . . . . . . . . . 21

2.4 .3 Factors Affecting Spinal Movements . . . . . . . . . . . . . . . . 21

2.5 Spine and Gaits . . . . . . . . . . . . . . . . . . . . . . 22

2.5.1 Functions of Spine Muscles on Gaits . . . . . . . . . . . . . . . . 22

2.5.2 Spinal Movements and the Associated Gaits . . . . . . . . . . . . . . . 23

2.6 Inspirations Extracted from a Biological Spine . . . . . . . . . . . . . . . 23

3 Effect of the Shape of the Spine on Spine-Driven Locomotion 25

3.1 Development of a Biologically Inspired Spine . . . . . . . . . . . . . . . . . 25

3.2 The Role of a Spine and the Effect of its Morphology on Locomotion . . . . . . . . 26

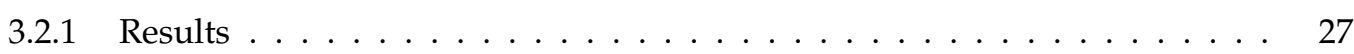

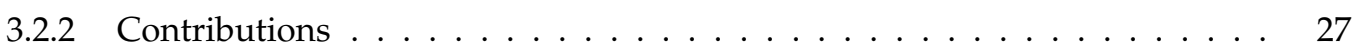

4 Embodiment Enables Spine-Driven Locomotion $\quad 29$

4.1 Results ........................... 30

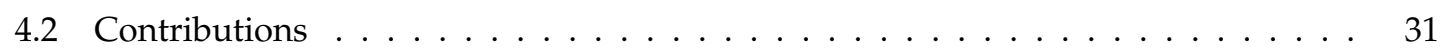

5 Effect of the Number of Spinal Joints on Spine-Driven Locomotion 33

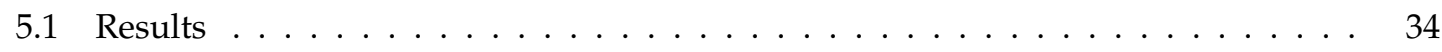

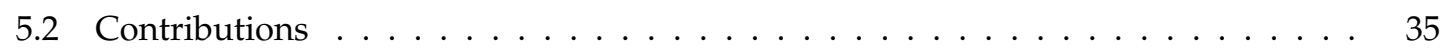

$6 \quad$ Flexible Spine as a Computational Resource $\quad 37$

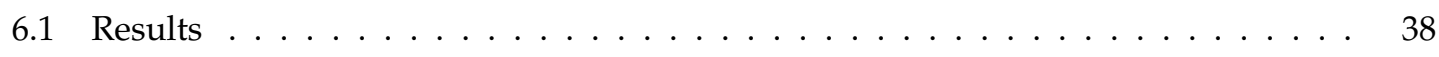

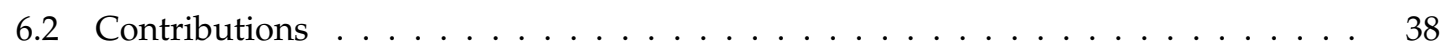

7 Effect of Spine Actuation and Stiffness on Locomotion $\quad 39$

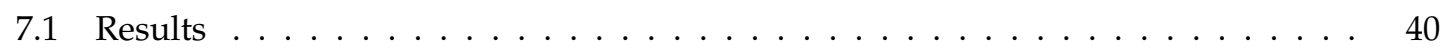

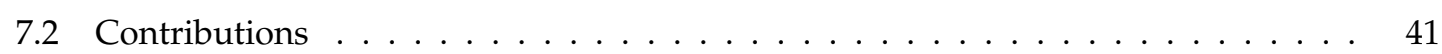

8 Discussion and Conclusion $\quad 43$

8.1 Summary of Results . . . . . . . . . . . . . . . . . . . . 43

8.2 General Implications . . . . . . . . . . . . . . . . . . . . . . 4 47

8.2.1 Implications for the Spinal Engine . . . . . . . . . . . . . . . 47

8.2 .2 Implications for a Biological Spine . . . . . . . . . . . . . . . . . . . . . . . . . . . . . . . . . . .

8.2.3 Implications for Robots with a Rigid Spine . . . . . . . . . . . . . . . . . . 48

8.3 Directions for Future Research . . . . . . . . . . . . . . . . . . . . . 48

8.3.1 Improvement of the Renny Robot . . . . . . . . . . . . . . . . . 49

8.3.2 Energy Efficiency of the Renny Robot . . . . . . . . . . . . . . . . . . 49

8.3.3 Energy Transfer and Store by a Spine . . . . . . . . . . . . . . . . 50 
8.3.4 Accuracy of the Spine Reservoir . . . . . . . . . . . . . . . . 50

8.3.5 Gait Switching based on Morphological Computation . . . . . . . . . . . . . 51

8.4 Conclusion ........................... 51

A The Effect of Morphology on the Spinal Engine Driven Locomotion in a Quadruped Robot

B Embodiment Enables the Spinal Engine in Quadruped Robot Locomotion

C Spine as an Engine: Effect of Spine Morphology on Spine-Driven Quadruped Locomotion

D Spine Dynamics as a Computational Resource in Spine-Driven Quadruped Locomotion 93

E The Effect of Spine Actuation and Stiffness on a Pneumatically-driven Quadruped Robot for Cheetah-like Locomotion

F Curriculum Vitae 


\section{List of Figures}

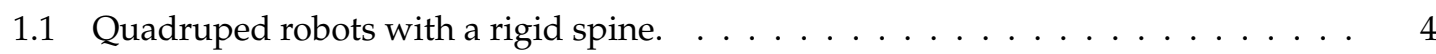

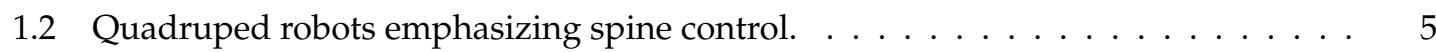

1.3 Existing quadruped robots with a tunable actuated spine. . . . . . . . . . . . 6

1.4 Design architecture for a biologically inspired robot . . . . . . . . . . . . 10

1.5 Snake locomotion . . . . . . . . . . . . . . . . . . . . . . . 12

1.6 Overview of the thesis. . . . . . . . . . . . . . . . . . . . . . . . . 12

2.1 Two basic models of legged locomotion. . . . . . . . . . . . . . . . . . . . . . 16

2.2 The evolution of spinal movements of vertebrate animals. . . . . . . . . . 16

2.3 Skeleton of a cheetah. . . . . . . . . . . . . . . . . . . . . . 18

2.4 Components of a spine. . . . . . . . . . . . . . . . . . . . . . . . . . . . 19

2.5 Instantaneous axes of rotation for the lumbar vertebrae. . . . . . . . . . . . . . 20

2.6 Description of reference planes. . . . . . . . . . . . . . . . . . . 20

3.1 Developed spinal structures. . . . . . . . . . . . . . . . . . . . . . . . . 26

\section{List of Tables}

2.1 Locomotor function of epaxial and hypaxial muscles in galloping, walking, and trotting gaits [Schilling and Carrier, 2010; Fife et al., 2001]. . . . . . . . . . 23

3.1 Similarities in morphology and function between a biological spinal structure and its artificial counterpart. . . . . . . . . . . . . . . . 26 


\section{Chapter 1}

\section{Introduction}

\subsection{Motivation}

Over the past decades, it has been widely accepted by most biologists as well as robotics researchers that locomotion is generally achieved by the coordination of the legs while the spine only facilitates locomotion by providing additional stability and energy in fast locomotion [Alexander, 1988]. Therefore, a considerable amount of research has been conducted on legged robots, but little effort has been put on the study of their spine. Most of the existing quadruped robots are very similar in their morphology in the sense that they are equipped with four actuated legs and a single rigid body [Poulakakis et al., 2005; Buchli and Ijspeert, 2008; Fukuoka et al., 2010; BostonDynamics, 2012; BostonDynamics, 2013a]. Some of them can perform well in rough terrains, especially BigDog robot, which can even walk on ice, but they need precise calculation at every time step, which therefore leads to high computational load. Furthermore, the resulting locomotion behavior is still much more constrained than their natural counterparts in terms of speed, energy efficiency, maneuverability, and adaptivity to rough terrain.

A major difference between these robots and animals is the spine. Such a spine is central to control body posture, provide the foundation to produce the leg movements, and integrate the leg and the spine actions [Schilling and Carrier, 2010]. A well known example is the cheetah, the fastest animal on land, which is able to reach up to $110 \mathrm{~km} / \mathrm{h}$. The pronounced spinal flexionextension movements make extensive body articulation, leading to great power and speed.

From an evolutionary point of view, it is natural to consider the spine as a propulsive engine of the vertebrate body to maintain a central role in locomotion in all craniates [Schilling and Carrier, 2010]. It was reported that the anatomy, the associated functions, and the resultant postures of a spine are evolved when animals left water and ventured into land. For example, a new function of the spine that emerged was to stabilize the trunk. Nevertheless, the locomotor function of the spine has never been replaced over time and it is still prevailing in locomotion. Based on mathematical simulations and analyses, Gracovetsky has proposed a biological hypothesis called "Spinal Engine", which emphasizes the locomotor role of the spine in human locomotion. 
This concept was then extended to quadruped animals featuring spinal flexion-extension movement [Gracovetsky and Iacono, 1987; Gracovetsky, 1989]. Additionally, Boszczyk systematically examined the anatomy of the mammalian lumbar spine and concluded that all examined spines suggest an exact accommodation to specific biomechanical demands, i.e., spine morphology depends on locomotion mode in nature [Boszczyk et al., 2001].

All of these biological findings imply that a spine might play a dominant role in locomotion. Nevertheless, there have been only a few attempts with limited success to implement an actuated spine to a quadruped robot, while substantial effort has been put in the design and optimization of the leg morphology and the associated controller.

In order to further improve quadruped robots' performance, using insights from biology, we aim to investigate how a spine contributes to locomotion and how its morphological parameters affect locomotion performance by building quadruped robots with an actuated spine.

\subsection{Contributions of a Spine to Legged Animal Locomo- tion}

In this section, we learn from animals (e.g., cheetah, pika) by summarizing the advantages of pronounced spinal flexion-extension movements on locomotion. Each advantage will be addressed from a biological point of view about how a spine contributes to locomotion.

\subsubsection{Speed}

It is known that cheetahs are the fastest land animals. They exhibit pronounced spinal flexionextension movements during their hunt. It was reported that these pronounced spinal movements contribute to the speed mainly by: (1) increasing the swing of the limbs, thus increasing the distance covered during suspended phases and the duration of the supported phases; (2) advancing the limbs more rapidly; (3) contributing to increased maximum forward extension of the limbs, which permits their greater backward acceleration before they strike the ground; (4) moving the body forward in measuring-worm fashion; and (5) reducing the relative forward velocity of the girdles when their respective limbs are propelling the body [Hildebrand, 1959] .

\subsubsection{Maneuverability}

It has been reported recently that a cheetah's hunting success is greatly attributed to its maneuverability, such as the impressive acceleration, deceleration, and the ability to change direction rapidly [Wilson et al., 2013]. During rapid deceleration, its flexile spine can extend the pitch limit of the body to provide stability. Moveover, the spine allows a cheetah to quickly twist and turn due to its flexibility as the prey struggles to escape from its claws. 


\subsubsection{Stability}

Stability is a key characteristic of locomotion especially when animals are escaping or hunting. A stable locomotion can gain great advantages in speed and maneuverability.

Hackert et al proposed that the flexion of the spine in small mammals and the associated forward displacement of the center of mass form a way to adjust the angle of attack of forelimbs [Hackert et al., 2001; Hackert et al., 2006]. This adjustment allows for a mechanical selfstabilization mechanism (i.e., mechanical stability without sensory feedback). Other studies revealed that the flexion of the spine enables the change of the spine's mass distribution, which also benefits self-stabilization of the body [Hackert, 2002].

Studies on the function of the cat lumbar spine indicated that during walking and trotting gaits, the epaxial muscles and abdominal musculature may act to stabilize the pelvic girdle and the trunk, while during galloping and half-bounding gaits, these muscles have the function as elastic elements to increase step length and limb speed [English, 1980].

\subsubsection{Energy Storage}

It is generally agreed that the spine is important to store energy in galloping quadruped animals [Cavagna et al., 1977; Taylor, 1978; Minetti et al., 1999; Hackert, 2002; Alexander, 2002]. From influential studies on the function of spine elastic structures in galloping gait of deer and dog [Alexander et al., 1985; Alexander, 1988], it was concluded that an aponeurosis in the back has a similar role as the tendon elasticity in the running gait of large mammals, working as a strain energy storage element. More specifically, internal kinetic energy which is lost by the body as the forelegs end their backward swing and the hind legs end their forward swing, is stored briefly as elastic strain energy, and recovered in an elastic recoil. Such an aponeurosis in the spine saves energy, and thus makes galloping the most economical gait at high speed.

There is a lot of evidence that a biological spine contributes greatly to locomotion, due to all of the above mentioned advantages. Therefore, it is worthwhile to implement a biologically inspired spine in the physical robots to explore its role and effect on locomotion and further improve the robots' performance.

\subsection{Early Attempts to Design Quadruped Robots}

With the advantages of a spine on locomotion being understood, it is interesting to see how these advantages can be achieved by building quadruped robots with an actuated spine and how such a robot outperforms the one with a rigid spine.

In this section, recent advances in robots with different configurations of the spine (e.g., rigid, passive, or actuated spine) are reviewed. 

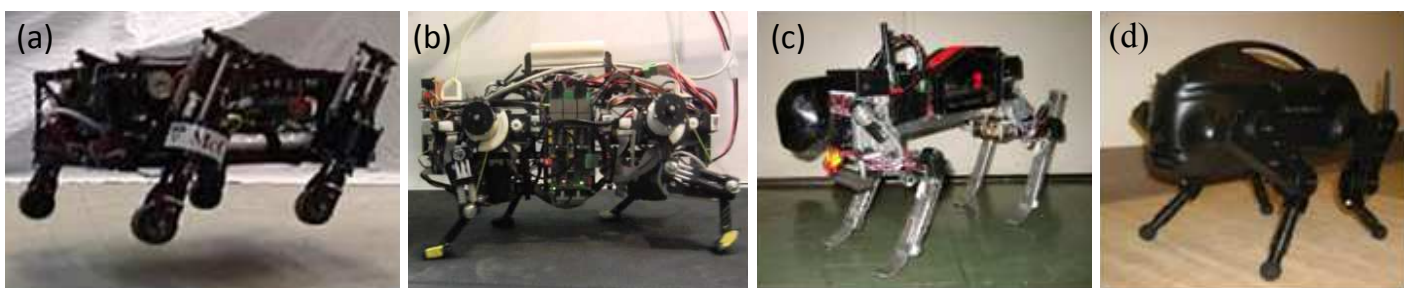

Figure 1.1: Quadruped robots with a rigid spine. (a) Scout robot; (b) Oncilla robot; (c) Tekken robot; (d) LittleDog robot.

\subsubsection{Robots with a Rigid Spine}

Legged robots have dominated the field of mobile robots, due to their ability to adapt to environment and terrain, for instance, Scout (Fig. 1.1(a)) developed by McGill University [Poulakakis et al., 2005]; Oncilla (Fig. 1.1(b)) by EPFL [Buchli and Ijspeert, 2008]; Tekken (Fig. 1.1(c)) by KIT [Fukuoka et al., 2010]; and LittleDog (Fig. 1.1(d)) by Boston Dynamics [BostonDynamics, 2012].

These robots only have a rigid structure as their spine, which contribute a little to locomotion. They can walk well, especially LittleDog in rough terrain. However a controller which has to do precise sensing, actuator control and communications is usually required in these robots, which leads to a high computational load. Their performance could be increased by introducing an actuated spine which can offer the robot more energy and freedom.

\subsubsection{Robots Emphasizing Control on a Simple Spinal Joint}

During the past decades, some researchers have come to realize the importance of an actuated spine on locomotion. For example, an actuated spine with three degrees of freedom was employed in the GEO robot (Fig. 1.2(a)) [Lewis and Bekey, 2002]. In this study, much attention was paid to the locomotor controller design that uses central pattern generators based on the adaptive ring rule model and adaptive modules and two basic reflexes (postural control and foot extension reflex). This controller allows GEO to quickly acquire a basic trot gait within minutes of inception.

SLAIR (Fig. 1.2(b)) is a modular multi-legged walking robot with an articulated body and force controlled legs [Palis et al., 2005]. The modular design and the control system of this robot enables it to easily extend more additional capabilities, e.g., climbing the stairs, overcoming obstacles.

The Whegs series of robots (Fig. 1.2(c)) use an actively controlled body joint which allows for sagittal spinal movements [Schroer et al., 2004; Allen et al., 2003; Boxerbaum et al., 2008]. These robots are able to pitch their bodies up to enable their front legs to reach higher and flex their bodies down to allow their front legs to maintain contact with the ground in irregular terrains by actuating this spinal joint.

In the early work of the MIT leg lab, the effect of an articulated spine was studied in a planar quadruped robot (Fig. 1.2(d)) [Leeser, 1996]. A finite state machine bounding controller was de- 

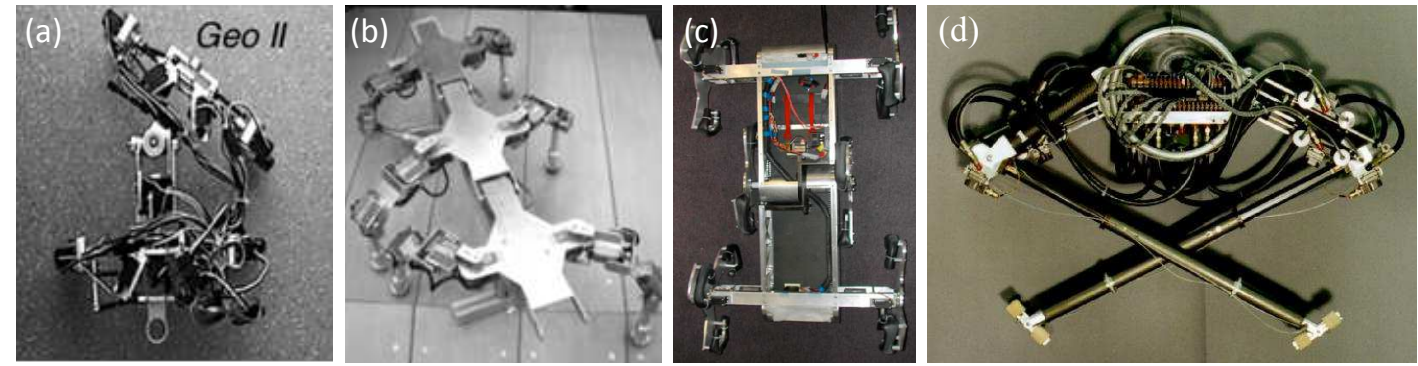

Figure 1.2: Quadruped robots emphasizing spine control. (a) GEO robot; (b) SLAIR robot; (c) Whegs II robot; (d) Planar quadruped robot.

signed to replicate the movements of a cheetah. It was concluded that thrusting with the back can be used to augment the thrust provided by the legs. Additionally, experiments suggested that the motion of the back can be used to modify the impedance characteristic of the legs.

The effect of an actuated spine and the strategy to control spinal movements in a quadruped robot were also investigated by simulation. For instance, Culha et al. developed a new quadruped robot model with an actuated spinal joint and proposed a bounding controller [Culha and Saranli, 2011]. This study revealed that the actuated spinal joint and its associated controller increase stride length, resulting in greater horizontal speed and higher hopping of the robot body.

In short, the design of the controller was studied in all of these above mentioned robots, but their spine was designed in such a simple way that only one simple revolute joint with one or two degrees of freedom is employed to connect and rotate the front and rear part of the body. This design is much simplified, compared with a biological spine regarding anatomical structure and morphology.

\subsubsection{Robots with a Focus on Spine Morphology}

Embodiment suggests that robots designed by clever morphology, e.g., proper use of materials, are usually simpler, more robust and adaptive than those based on the classical control paradigm [Pfeifer and Bongard, 2007; Pfeifer et al., 2007]. Furthermore, from a biological point of view, the morphology of mammalian lumbar spine suggests an exact accommodation to specific biomechanical demands [Boszczyk et al., 2001]. Inspired by these ideas, many researchers have started to consider the morphology as one of the main focuses.

For example, Mizuuchi et al. developed a series of robots with a flexible spine, starting with a quadruped robot called SQ43 (Fig. 1.3(a)). In this SQ43 prototype, the spine consists of soft memory foams and hard vertebrae which are connected together by a wire driven by the motor [Mizuuchi et al., 1999]. The motor can control the posture and stiffness of the spine by pulling the wire. The employment of such an articulated spine increases its ability to absorb shock and to work in various environments. Additionally, the flexibility of the spine and the diversity of the posture can be observed, due to this actuated flexible spine. 

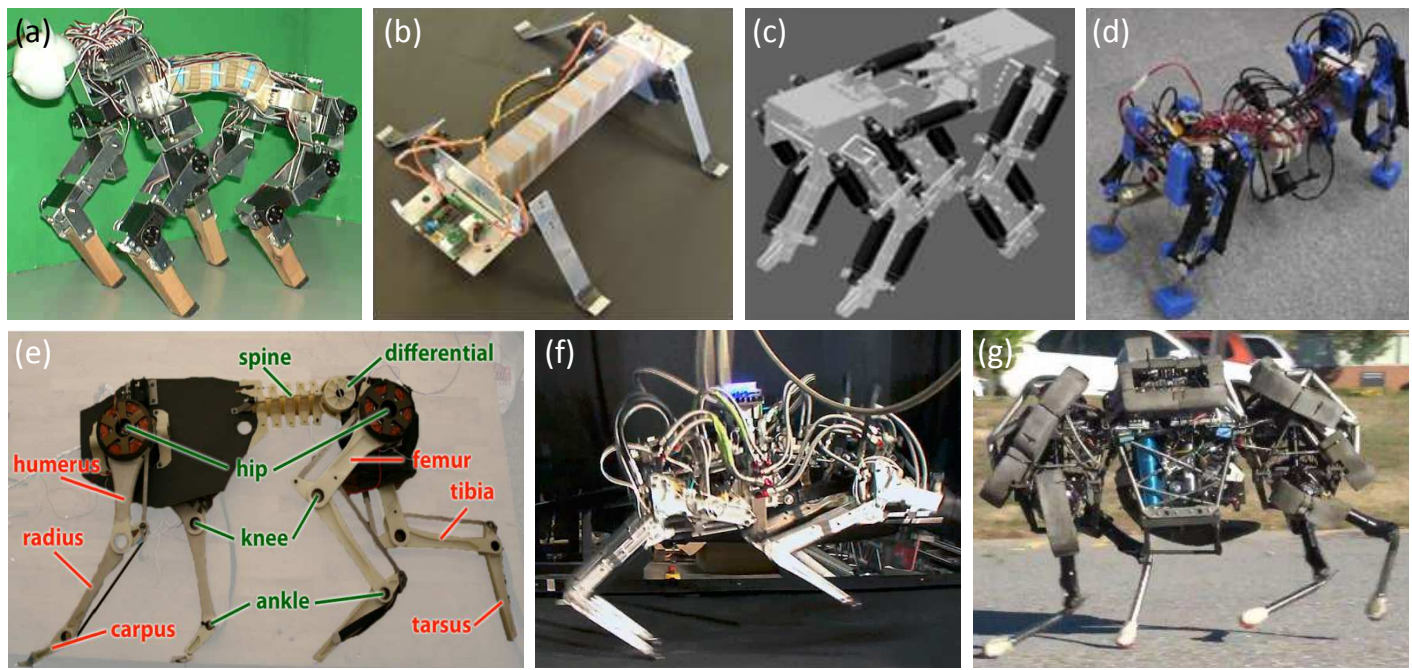

Figure 1.3: Existing quadruped robots with a tunable actuated spine. (a) SQ43 robot; (b) A robot featuring tunable spine stiffness by Osaka Institute of Technology; (c) A pneumatically-driven robot by Osaka Institute of Technology; (d) A robot featuring tunable spine stiffness by Tohoku University; (e) Cheetah robot by MIT; ( $f$ ) Cheetah robot by Boston Dynamics; (g) Wildcat robot by Boston Dynamics.

The effect of tunable spine stiffness on locomotion was studied in a robot with a passive spine (Fig. 1.3(b)). It was observed that there are several interactions between the gait pattern and the viscoelasticity of the spine that can achieve various types of successful locomotion [Takuma and Masuda, 2010]. However, the resultant speed is rather slow in this study.

Tsujita et al. developed a pneumatically-driven robot with an actuated spinal joint driven by antagonistic pairs of artificial muscles (Fig. 1.3(c)) and introduced a new controller. This robot can generate smooth gait transition by changing locomotion speed and muscle tone [Tsujita et al., 2008]. Furthermore, the effect of spine stiffness on the stability of locomotion in crawl, trot and pace patterns was qualitatively studied [Tsujita and Miki, 2011].

Similarly, gait transition between trotting gait and bouncing gait was also investigated in another quadruped robot with tunable spine stiffness (Fig. 1.3(d). The results showed that gait transition can be achieved smoothly by appropriately tuning the stiffness of the spinal joint [Sakai et al., 2007].

Recently, new and powerful robots have been developed by MIT and Boston Dynamics, aiming to break through the limitation of existing robots partially by embedding an actuated spine. In the Cheetah robot developed by MIT (Fig. 1.3(e)), a tendon-driven spine is connected to the rear hip motors through a differential gear to coordinate itself and legs. This design improves energy efficiency by acting as a parallel spring for the rear legs [Folkertsma et al., 2012].

Another Cheetah robot (Fig. 1.3(f)) developed by Boston Dynamics is the fastest legged robot so far, surpassing $29 \mathrm{mph}$, a new land speed record for legged robots. It has an articulated spine 
that flexes back and forth on each step, increasing its stride and running speed, much like the animal does [BostonDynamics, 2013b]. However, this robot is tethered to a power supply on a treadmill. Therefore, WildCat robot (Fig. 1.3(g)), untethered, has been developed as the second generation of Cheetah robot with the aim to run fast on all types of terrain. It can run at about $16 \mathrm{mph}$ on flat terrain using bounding and galloping gaits. Enabled by the articulated spine and biomimetic stride, these two gaits are smooth, as is its ability to turn. Unfortunately, more detailed technical information on Cheetah and WildCat still remains confidential. Nevertheless, we believe that researchers must deeply investigate morphological parameters of the spine and choose the optimal ones based on the biological inspiration or experimental experience.

The robots introduced in this section cover the key research in the field of quadruped robots with an actuated spine. They outperform some early robots partially by employing optimal spine morphology inspired by biology.

However, when taking the anatomical structure of a biological spine into account, we realize that there are still other important spinal morphological parameters that are worth investigating further. Such an investigation will provide invaluable information about the locomotive function of the spine and its morphological effect on locomotion. Moveover, it will help us to extract design principles to improve or optimize the existing robots' performance. Based on these main motivations, I proposed several research questions and hypotheses which will be elaborated in the next section.

\subsection{Research Questions and Hypotheses}

This thesis aims to investigate the locomotion mechanism by taking the spine as the main object. Five specific research questions and associated hypotheses are derived from this main goal.

Many discoveries suggested that the spine may play a critical role in vertebrate locomotion [Schilling and Carrier, 2010; Schilling, 2011; Gracovetsky, 1985; Gracovetsky, 1989]. This view is further supported by the evolution of the animals which suggested that all of them might descend from ancient fishes featuring pronounced spinal movements. However it is unclear to which extent the spine benefits to locomotion. Therefore, our first and the most important question is:

Research Question 1: Whether a spine is possible to be an engine for vertebrate locomotion?

Our hypothesis is that the spine and its surrounding tissues comprise the basic engine of locomotion. We investigate whether this hypothesis is applicable to vertebrate locomotion. Therefore, studies on non-vertebrate animals are beyond the scope of this thesis.

This hypothesis will be validated by building robots in which the actuated spine is employed while the legs are fixed (e.g., the Kitty robot, the simulation model). We believe that it is a simple and straightforward way to test this hypothesis, because it captures the most important morpho- 
logical features (e.g., an actuated spine) and ignores less important ones (e.g., the actuation of legs).

If the first research question holds true, it is natural to ask how the spine drives and affects locomotion. To address this question, it is crucial to gain knowledge of the anatomy, the spinal movements, the relation between spine and gaits of a biological spine first, and then extract ideas and hypotheses from them. The second question is therefore:

Research Question 2: What are the key factors of a biological spine to affect quadruped locomotion?

Our hypothesis is that some morphological parameters of a spine, such as the components, the motion range, and the stiffness distribution of a spine, have great influences on spine-driven locomotion and they can be extracted as design principles to guide the design of a robot. Inspirations obtained from biology will be presented in detail in Chapter 2 .

After acquiring ideas from biology, we move on to implement them to robots to check if the experimental results are consistent with these hypotheses. In other words, the inspirations provided by the biology will be tested by building robots. Therefore, the third question is:

Research Question 3: How the spine morphology affects spine-driven locomotion in robotic platforms?

Our hypothesis is that fast and stable locomotion can be achieved by appropriately making use of intrinsic properties of a spine. The clues to appropriately design such spine properties come from inspirations summarized in Chapter 2, and thus the hypotheses are formulated as follows.

Hypothesis 3.1: The shape of a spinal column has an effect on locomotion.

Hypothesis 3.2: A model with one actuated spinal joint is capable of generating bounding, trotting gaits and turning behavior.

Hypothesis 3.3: The rear position of a spinal joint benefits locomotion by providing the rear legs with more freedom to swing further.

Hypothesis 3.4: A robot with a spine of which the dorsal stiffness is higher than the ventral stiffness runs faster than the one with other configurations.

Hypothesis 3.5: It is sufficient for a robot with two spinal joints to produce more dynamic behaviors.

Hypothesis 3.6: A spine with compliant elements stores and transfers energy.

The first research question aims to validate the spinal engine hypothesis from an anatomical and a mechanical point of view. One might ask whether this hypothesis holds true in the perspective of control. It is known that a proper design of morphology and selection of materials can significantly reduce the complexity of the required control algorithm, which is termed morphological computation [Pfeifer and Bongard, 2007]. Further theoretical studies reported that a soft body could be a potential computational resource to partially take over the control tasks due to its 
inherent elasticity and nonlinearity in the context of morphological computation [Hauser et al., 2011; Hauser et al., 2012]. More specifically, this soft body together with a simple readout, is able to emulate complex mappings from input to output streams in continuous time. Therefore, the fourth research question is:

Research Question 4: Is it possible to use a compliant spine as a computational resource to drive locomotion?

Our hypothesis is that a compliant spine structure is not only a mechanical component connecting the front and rear legs, but also a computational resource to drive locomotion, because such a spine consists of a sequence of soft silicone blocks which are endowed with the properties of elasticity and nonlinearity.

When quadruped animals move, they need to coordinate the spine and leg movements to achieve fast and stable locomotion, so the last research question is:

Research Question 5: How to coordinate the movements of a spine and the legs?

Our hypothesis is that the movements of a spine are closely correlated to that of legs to achieve stable and fast locomotion. However the way to coordinate them is unclear and will be investigated.

\subsection{Research Methodology}

To address the research questions and hypotheses outlined in the previous section, we apply the following research methodologies and theoretical tools in this thesis.

\subsubsection{Biorobotics}

Biorobotics is a methodology of building robots to evaluate biological hypotheses or mimic the behaviors of biological organism [Webb, 2002; Meyer and Guillot, 2008; Trimmera, 2008]. To design robots comparable to biological models, robotics researchers are increasingly searching for inspirations and design clues from biology. Figure 1.4 illustrates the general procedure of biologically inspired robot design. We first identify problems, then extract information from biological study, and finally implement it to robots. Afterwards, we use engineering tools or mathematical tools to verify these biological hypotheses. If experimental results are inconsistent with a hypothesis, then this hypothesis needs to be modified or the design of the robot needs to be improved.

Biomechanics research of animals provides an invaluable source of ideas for robot design but the process of implementation involves great complexity. A direct implementation of biological features and morphology is often ineffective and misleading engineers due to two reasons. First, engineers investigate animals to achieve a few particular functions whereas features of animals may serve multiple functions or often their roles remain unknown. Second, the difference 


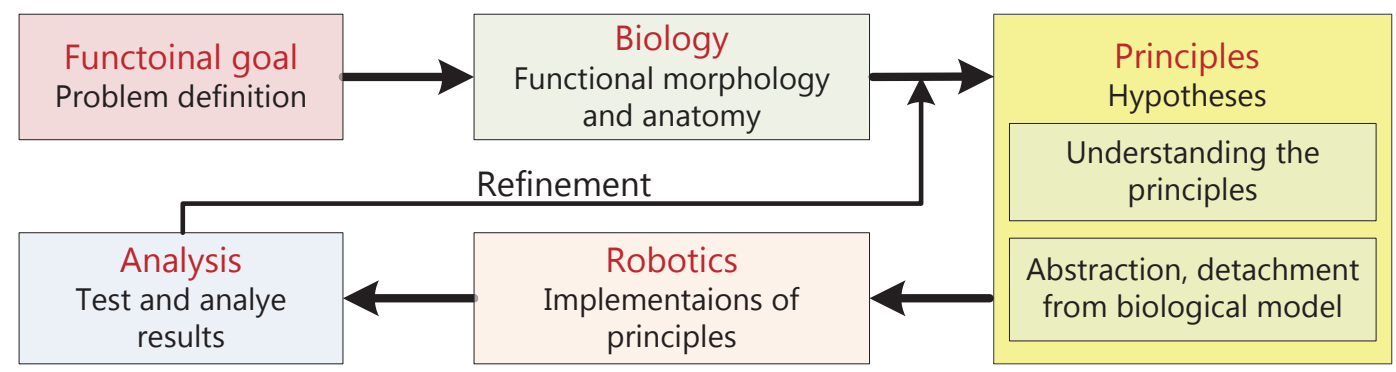

Figure 1.4: Design architecture for a biologically inspired robot

between an engineering manufacturing process and biological synthesis causes the difficulties in direct replication. Therefore, it is of great importance to simplify biological inspiration and extract fundamental principles before moving on to engineering manufacturing process.

\subsubsection{Synthetic Approach}

Synthetic approach is applied to investigate the spine-driven locomotion throughout this thesis. Instead of analytically decomposing a specific biological system, we strive to understand the behaviors of natural systems by building robotic models, because this approach helps to understand natural forms of intelligence, abstract general principles of intelligent behavior that hold not only for biological systems but for behaving systems in general, and build intelligent artifacts [Pfeifer and Bongard, 2007; Pfeifer et al., 2007].

Therefore, several robotic platforms were developed to study how the spine drives locomotion. A number of different spine morphologies were manufactured and tested in terms of their locomotion performance.

\subsubsection{Information Theory}

The embodied view suggests that the behavior of a robot is generated from the reciprocal and dynamical coupling between the control, the body, and the environment [Pfeifer et al., 2007; Pfeifer and Bongard, 2007], but how the change of spinal morphologies and environments modulates this embodied coupling is still unknown. Such an investigation is challenging because this coupling is usually nonlinear and complex. One promising approach is to take information theoretic measures to study and characterize how embodiment actively promotes information processing by exploiting the dynamics of the interaction between an embodied system and its environment. For example, the changes of the behavior of the robot can be characterized by the changes of this coupling regime. This is because the information theoretic approach is intrinsically a model-free approach and can be used to detect nonlinear relations between variables. Thus, it has been recently used to characterize the coupling regime of robots [Nakajima et al., 2011; Sumioka et al., 
2010; Lungarella and Sporns, 2006; Lungarella et al., 2005].

\subsubsection{Morphological Computation}

A well designed mechanical system allows us to significantly reduce the complexity of the required control algorithm as well as to increase the robustness against the environmental perturbation [Pfeifer and Bongard, 2007]. There are many examples of robots which require much less control if stiffness and deformability of materials are used properly. For instance, locomotion can be facilitated by applying soft elements (e.g., springs, artificial pneumatic actuators) to robots which use a simpler controller [Iida et al., 2009; Sheikh, 2013; Hosoda et al., 2008]. This implies that part of the computation needed for control can be outsourced to the body by properly using morphological properties. In this sense, a compliant body could be a potential computational resource.

This hypothesis, usually referred to as morphological computation (MC), has recently received theoretical supports by Hauser et al. [Hauser et al., 2011; Hauser et al., 2012]. They proposed theoretical models for MC with a compliant body, and demonstrated how such a compliant physical body can be potentially used as a computational resource. In these models, the concept of reservoir computing is applied to a random network of mass-spring system. Instead of using a neural network or a network of leaky integrators (as in standard approaches for reservoir computing), MC models employ a compliant physical body as a reservoir. This theory suggests that a complex physical body could be a potential computational resource, due to its elasticity and nonlinearity inherently embedded in itself. Moreover, it was demonstrated how static feedback from the sensors into the physical body (via actuators) can be used to generate autonomously periodic patterns, e.g., locomotion [Hauser et al., 2012] .

\subsection{Issues Not Considered in this Thesis}

It is straightforward to test spinal engine hypothesis in snakes and limbless lizards. Let's take a snake as an example. A snake uses at least five unique modes of terrestrial locomotion. They are lateral undulation, sidewinding, concertina, rectilinear, and slide-pushing (Fig. 1.5). These modes are driven by the spine which has between 200-400 vertebrae. That is what makes them so flexible and helps them move along. However, a snake's spine can not achieve sagittal bending, which is a characteristic of advanced mammals [Schilling and Hackert, 2006]. Such a feature in vertebrate animals was developed later than lateral bending and axial rotation [Gracovetsky, 1989]. It is correlated with fast gaits, e.g., galloping, bounding [Pridmore, 1992]. Since we are only interested in the fast animals and gaits, a snake is not the studying object in this thesis.

A tail is part of a spine. More precisely, it is an extension of a spine. Recent studies unveiled that a tail plays a crucial role in locomotion. For example, it was demonstrated that a gecko's 
(a)

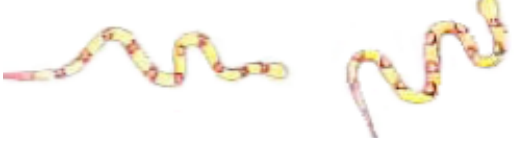

(c)

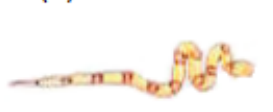

(d)

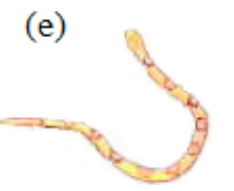

Figure 1.5: Snake locomotion: (a) lateral undulation; (b) sidewinding; (c) concertina; (d) rectilinear; (e) slide-pushing. Note this figure is adopted from [Moon, 2001].

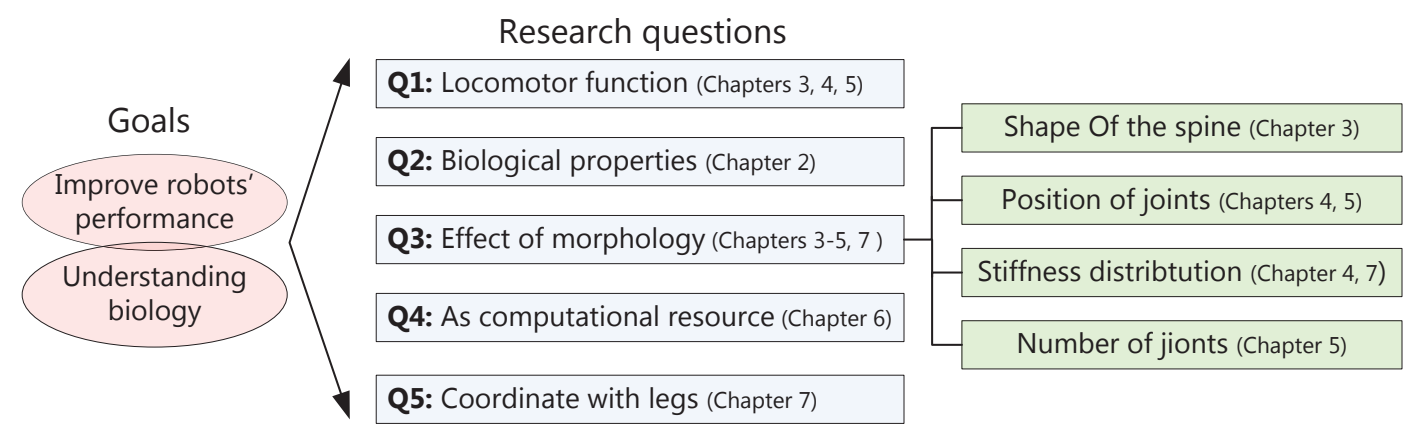

Figure 1.6: Overview of the thesis. There are two goals of this thesis: First, to improve robots' performance by employing ideas and principles from biology; Second, to better understand biological systems by using robots as models of specific animal systems to test biological hypotheses.

tail functions as an emergency fifth leg to prevent falling during rapid climbing [Jusufi et al., 2008]. It was also reported that a lizard controls the swing of its tails in a measured manner to redirect angular momentum from its body to its tail, stabilizing body attitude in the sagittal plane [Libby et al., 2012]. In order to gain comprehensive understanding of the function of a tail in addition to that of the spine, one need to design a more complicated robot to explore the proper tail morphology first and then investigate the way to control it. This is beyond the scope of this study, but certainly worth further exploration.

\subsection{Overview}

This thesis is structured around five papers, which have been published in scientific journal, conferences, or workshop. The following chapters are organized into five main topics related to research questions and associated hypotheses: biological properties of a spine (chapter 2); the spinal engine (Chapters 3, 4, 5); the effect of spine morphology on locomotion (Chapters 3, 4, 5, 7); a compliant spine as a computational source (Chapter 6); and the coordination between legs and a spine (Chapter 7), as shown in Fig. 1.6.

In Chapter 2, a conventional legged locomotion theory is presented first. Then a hypothesis called "Spinal Engine" is introduced in an attempt to encompass all vertebrate locomotion. To test 
this hypothesis, the spine anatomy, the spinal movements, and the relation between the spinal movements and the gaits are studied. Furthermore, important spine morphological properties are extracted. This chapter provides biological inspirations and serves as the basis and starting point of further investigation in the subsequent chapters.

In Chapter 3, important features of a biological spine extracted in Chapter 2 are implemented to an artificial spine. Then this spine is employed to the Kitty robot which has no leg actuation. At the end, spine-driven locomotion is demonstrated and several spine morphologies are explored regarding the shape of the spinal column.

Chapter 4 is focused on the validation of the spinal engine hypothesis in the Kitty robot featuring an articulated spine. Additionally, the spine morphology differing in the position of the virtual spinal joint (i.e., a point where the spine is more likely to get bent) is explored through the dynamical interaction between the brain, the body, and the environment. Furthermore, information theory is taken to quantify how the change of spine morphology modulates this embodied coupling.

In Chapter 5, in addition to testing the spinal engine hypothesis in a simulated model, we investigate the effect of the number of spinal joints on locomotion. At the end, a biologically plausible spine model is proposed to emulate cheetah-like behaviors.

In Chapter 6, the possibility of a compliant spine to be a computational resource to drive quadruped locomotion is investigated in the Kitty robot. We also demonstrate the robustness of such a system against external perturbation.

Chapter 7 is focused on the method to coordinate legs and a spine. Therefore, a pneumatically driven robot called Renny with spine and leg actuation is developed and tested. Moreover, the actuation type and the stiffness distribution of the spine are investigated.

In Chapter 8, we conclude this thesis by summarizing and interpreting all results. Recommendations for future work are also proposed. 



\section{Chapter 2}

\section{Spinal Engine Hypothesis and Biological Properties of a Spine}

In this chapter, first, a conventional theory of legged locomotion is presented, including a brief description on its two basic mechanism models, its great advantages and limitations. Then the hypothesis called "Spinal Engine" is introduced, also aiming to understand the locomotion mechanism, but from a different perspective. To better understand this hypothesis, the spine anatomy, the spinal movements, and the relation between the spinal movements and the gaits are studied. Furthermore, important spine morphological properties are extracted, which helps to understand how the spine morphology affects locomotion and provides real robots with some design principles. Cheetahs are considered to be the main study object due to their fastest speed on land and pronounced spinal moments during the hunt.

\subsection{Hypothesis on Legged Locomotion}

Locomotion is generally perceived as being a function of the legs [Raibert, 2000; Alexander, 2003]. In this context, it is generally agreed that all animals exhibit remarkable similarity in their locomotion mechanism despite differences in the body size and the number of legs [Dickinson et al., 2000]. All of these animals use two basic mechanisms for minimizing energy expenditure for locomotion: inverted pendulum walking model (Fig. 2.1(a)) and spring-mass model (Fig. 2.1(b)).

When an animal walks, its body vaults up and over each stiff leg in an arc, similar to an inverted pendulum (Fig. 2.1(a)). This form is energetically economical because it allows for the exchange of gravitational potential and kinetic energy of the body. Kinetic energy in the first half of the stance phase is transformed into gravitational potential energy, which is partially recovered as the body falls forward and downward in the second half of the stance phase [Dickinson et al., 2000].

While bipeds are running, or other animals are using bounding, hopping, or galloping gaits, they change to the second mechanism to travel faster and energetically efficiently. Such a mecha- 

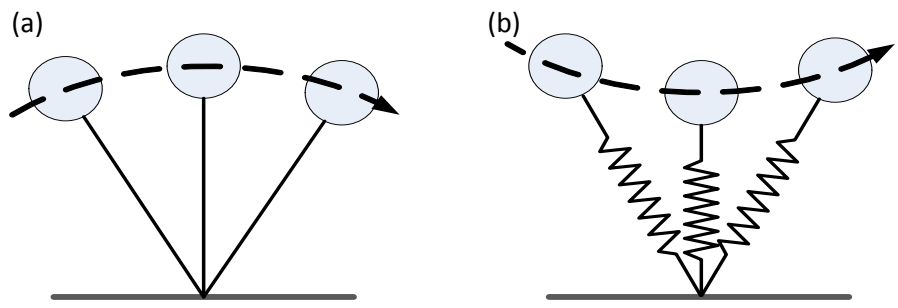

Figure 2.1: Two basic models of legged locomotion: (a) inverted pendulum in walking gait; (b) spring-mass model in running gait of biped, or bounding, galloping, and hopping gaits of other animals.

(a)

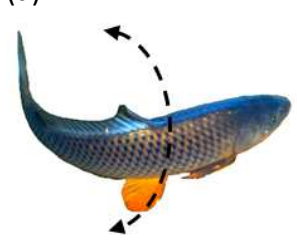

(b)

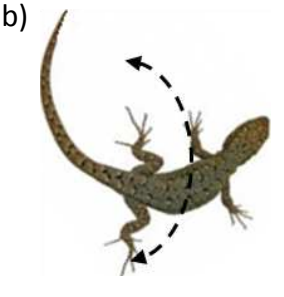

(c)

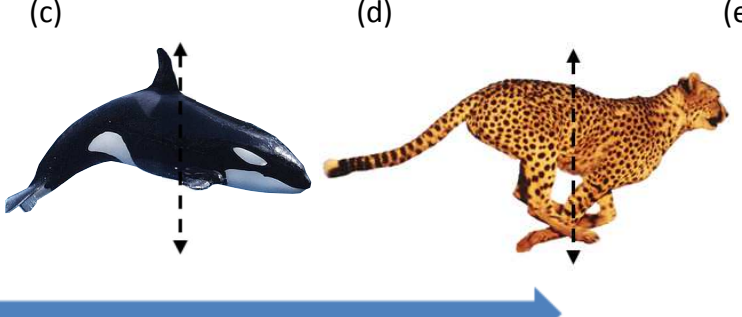

(d)

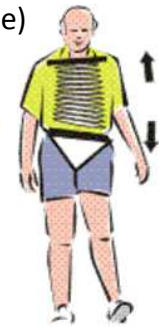

The evolution of vertebraete locomotion

Figure 2.2: The evolution of spinal movements of vertebrate animals. Dashed arrows represent the direction of spinal movements. (a) Lateral movements of the body and tail propel fish, but sagittal bending and rotation of the body are not present; (b) lateral movements of reptile animals on land; (c) movements of a whale which went back to the sea, but its terrestrial spinal flexionextension movements are still maintained; (d) movements of a cheetah featuring pronounced spinal flexion-extension movements; (e) movements of a human being, who evolved to use a counter rotation of the spine [Burgess and Practitioner, 2013], in addition to spinal flexion-extension movements.

nism is analogous to a spring mass system (Fig. 2.1(b)). As a leg strikes the ground in a running gait, kinetic and gravitational potential energy is temporarily stored as elastic strain energy in the leg muscles, tendons, and ligaments and then nearly all is recovered during the propulsive second half of the stance phase. Horizontal kinetic energy and gravitational potential energy fluctuate in phase [Dickinson et al., 2000]. The main difference between these two mechanisms is that elastic body elements are ineffective in walking gait.

Legged locomotion theory has been successful in explaining the locomotion mechanism of a wide variety of animals that differ in the number of legs, the body mass, or the skeleton type, including lizards, dogs, kangaroos, humans, etc [Dickinson et al., 2000]. However, we found that this theory is only applicable to the vertebrate animals that are endowed with legs. Therefore, it is necessary to propose a unified theory of vertebrate locomotion which could encompass all vertebrates with or without legs. 


\subsection{Spinal Engine Hypothesis}

It is the desire to investigate the essential of vertebrate locomotion that has encouraged some biologists to propose the biological hypothesis of spinal engine.

\subsubsection{Biological Concept of the Spinal Engine}

The spinal engine theory has been formulated and tested based on mathematical simulations and analyses [Gracovetsky, 1985; Gracovetsky and Iacono, 1987; Gracovetsky, 1989]. Such a hypothesis has been the subject of numerous criticisms ever since its birth, but never really been replaced. It emphasizes two points: First, locomotion is achieved by the motion of the spine and the limbs come after; Second, the spine and its local musculatures are the driving force behind any vertebrate's movement, from fishes, to lizards, to mammals, to human beings.

\subsubsection{Evolution of a Spine}

From an evolutionary point of view, it is natural to consider the spine as a propulsive engine of the vertebrate body which maintains a central role in locomotion in all craniates [Schilling and Carrier, 2010]. The presumed starting point of craniates is primitive fish (Fig. 2.2(a)), the ancestor of all tetrapods, whose locomotion is driven by spinal lateral bending movements generated by the spine. When fish ventured onto land, the vertebral column gradually evolved to solve the problems they were facing, e.g., gravity, obstacle, friction. Therefore, axial rotation evolved. This axial rotation movements combined with the lateral movements resulted in spinal flexionextension movements, which is one of the main striking characteristics of mammalian locomotion, including whales (Fig. 2.2(c)), cheetahs (Fig. 2.2(d)), and human beings (Fig. 2.2(e)). Some vertebrates, such as whale, dolphin, subsequently returned to the sea, while retaining their capacities for flexion-extension moments acquired during their stay on dry land. The ability to dorsoventrally flex and extend the body axis enables the evolution of fast gaits in mammals such as gallop or half-bound.

In short, the anatomy of the spine, the spinal movements, and the gaits have evolved to adapt to the new environment. For example, in a tetrapod with a sprawled limb posture (Fig. 2.2(b)), as a consequence of the evolution of supporting limbs and transition to land, the axial muscles function additionally to stabilize the trunk. Nevertheless, the locomotor function of the spine has never been replaced over time and it is still prevailing in locomotion. 


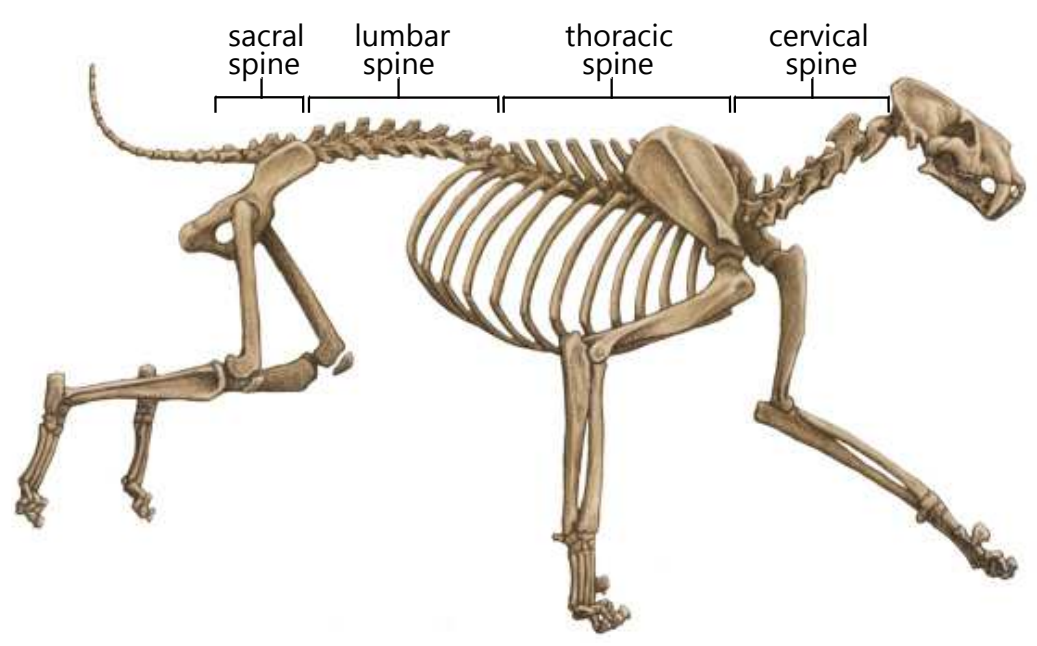

Figure 2.3: Skeleton of a cheetah. It consists of cervical, thoracic, lumbar, and sacral spine.

\subsection{Spine Anatomy}

The anatomy of the spine is usually described by dividing up the spine into four major sections. Each section is made up of individual bones called vertebrae. Ligaments and muscles connect these bones together to form the spinal column. The spinal column provides the main support for the body, allowing to bend and twist. Additionally, the spinal column holds and protects the spinal cord, which is a bundle of nerves that sends signals to other parts of the body.

\subsubsection{Sections of a Spine}

A spine consists of cervical, thoracic, lumbar, and sacral spines [Institute, 2013], as shown in Fig. 2.3. The neck section of the spine is known as the cervical spine which allows for a wide range of head movements instead of locomotion movements. The thoracic spine is more stable than the cervical or lumbar sections because of rib attachments and longer spinous processes. Additionally, the rib cage and ligament system limit the range of motion of the thoracic spine. The lumbar spine has a wider range of motion than the thoracic spine, thus allowing for significant flexion-extension movements but limiting rotation. The sacral spine refers to the five fused vertebrae at the end of the spinal column, so it cannot provide larger range of motion.

\subsubsection{Components of a Spine}

A spine consists of discrete bony elements, namely vertebrae, ligaments, intervertebral discs and zygapophyseal joints. The vertebrae are joined by passive ligaments and kept separated by intervertebral discs. The zygapophyseal joints are dynamically controlled by muscular activation. 


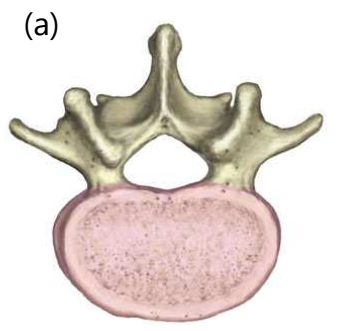

(b) intervertebral disc

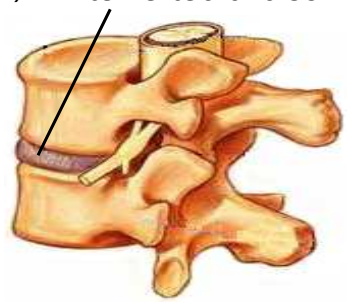

(c)

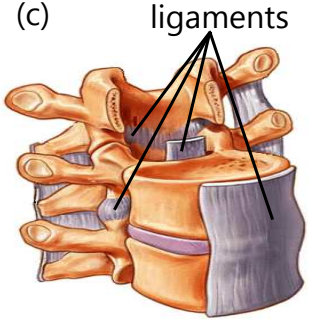

(d) zygapophysial joint

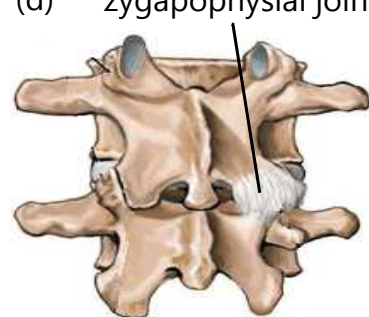

Figure 2.4: Components of a spine. (a) Vertebrae; (b) Intervertebral disc; (c) Ligaments; (d) Zygapophysial joint.

Vertebrae (Fig. 2.4(a)) are the highly specialized bones which collectively make up the spinal column. The number of vertebrae varies with the species of the animals from 10 in frogs to 56 in tigers. The bony elements create attachment points for muscles and other bones, allowing for flexible movement in a range of directions. All spinal movements are distributed over the connecting joints of these vertebrae.

Intervertebral discs (Fig. 2.4(b)) are located between the vertebrae, and firmly joined with the endplates of the vertebrae. They are morphologically structured soft tissue cushions serving as the spine's shock absorbing system, which protects the vertebrae and other structures (i.e. nerves). The discs allow for vertebral flexion-extension movements. Individual disc movement is very limited; however, considerable motion is possible when several disc movements are combined together.

Ligaments (Fig. 2.4(c)) are the fibrous, slightly stretchy connective tissues that hold one bone to another in the body. Ligaments control the range of motion of a joint and stabilize this joint so that the bones move in the proper alignment. Mechanically speaking, spinal ligaments behave as other soft tissues of the body and they are viscoelastic with nonlinear elastic responses [Provenzano et al., 2001].

A zygapophysial joint (Fig. 2.4(d)) is a movable joint between the superior articular process of one vertebra and the inferior articular process of the vertebra directly above it. The biomechanical function of each pair of zygapophysial joints is to guide and limit movements of the spinal motion segment. In the lumbar spine, the zygapophysial joints function to protect the motion segment from anterior shear forces, excessive rotation and flexion [Moore and Roy, 2011].

\subsection{Spinal Movements}

The lumbar spine is the main object when spinal movements are taken into account, because it both bears the most weight and allows the greatest amount of motion [NASS, 2013]. 

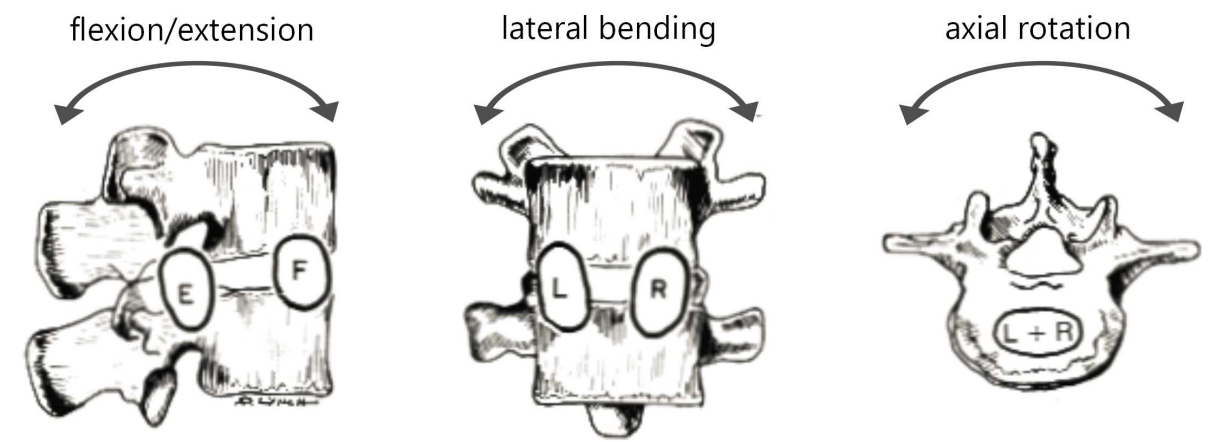

Figure 2.5: Instantaneous axes of rotation for the lumbar vertebrae. Note: this figure is adopted from [White and Panjabi, 1990].

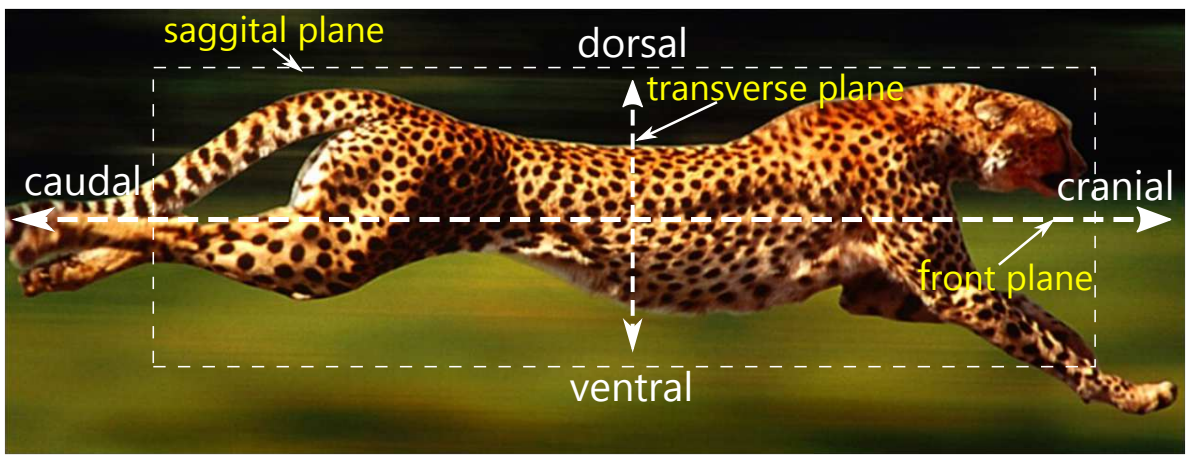

Figure 2.6: Description of reference planes.

\subsubsection{Type of Spinal Movements}

A spine can achieve flexion-extension, lateral bending and axial rotation movements as shown in Fig. 2.5. Figure 2.6 illustrates the planes used to describe the spinal movements.

- Flexion refers to bending forward about an axis perpendicular to the sagittal plane; extension refers to bending backward about this axis. Flexion-extension is also called the sagittal bending.

- Lateral bending refers to bending to either the left or right side.

- Axial rotation movements are the movements of the vertebra around an axis perpendicular to the transverse plane. All rotations produce a change in the orientation of the vertebrae.

Lateral bending occurs mostly in the upper lumbar motion segment. In the lower lumbar spine and lumbo-sacral region, flexion-extension movements are the primary motions. In contrast, axial rotation movements are limited in this region, compared to the cervical spine.

The spinal engine hypothesis states that flexion-extension is the result of the combination of lateral bending and axial rotation, from an evolutionary point of view [Gracovetsky, 1989]. One 
example is fish. When it came to shore it had appendages and its spine was capable of lateral flexion. In this case, its spine bent in the horizontal plane without inducing any axial torque. When fish ventured onto land, the need to clear obstacles necessitated the axial counter-rotation of the body. In the presence of lateral bending, together with axial rotation, the spine reacted by inducing an additional motion in the sagittal plane. Such an additional motion is referred to as sagittal bending, which is the coupled motion of lateral bending and axial rotation.

\subsubsection{Intervertebral Joint Movements}

It is known that all spinal movements are the results of small intervertebral movements that add up to what is called pelvic motion. In order to determine how the vertebral column is involved in spinal movements, intervertebral joint movements were studied in cat [Macpherson and Ye, 1998], horse [Townsend et al., 1983], and smaller mammals, e.g., pika [Schilling and Hackert, 2006]. These studies showed that the range of motion throughout the spine varies among the species, suggesting an exact accommodation to specific biomechanical demands. For example, a horse holds the back almost rigid, making its running action more efficient and thus leading to greater endurance. In contrast, a cheetah, exhibiting pronounced spinal movements, similar to that of a cat, is the fastest animal for a short dash [Hildebrand, 1959]. These marked spinal flexion-extension movements contribute to speed by increasing the swing of the limbs, advancing the limbs more rapidly, etc. The great difference in flexibility of a spine is caused by its anatomical structure. For a cat ${ }^{1}$ dorsoventral flexion and extension occurring in each intervertebral joint is around $10^{\circ}$ on average with some are above $20^{\circ}$ [Macpherson and Ye, 1998]. On the other hand, equine is around $4^{\circ}$ on average, and only one exceeds $20^{\circ}$ [Townsend et al., 1983]. As a result, between a horse and a cheetah there is a $70^{\circ}$ difference in the angle that the scapula makes with the pelvis during running. $130^{\circ}$ for a cheetah and $60^{\circ}$ for a horse.

\subsubsection{Factors Affecting Spinal Movements}

A spine, when considered as a whole, comprises multiple vertebrae with a variety of movements. The range of the spinal movements is controlled by the coordinated actions of the spinal muscles and constrained by the passive ligaments (Fig. 2.4(c)) connecting the vertebrae [Hansen et al., 2006]. The presence of the asymmetrical spinal movements and stiffness is partially due to the uneven distribution, elasticity, and transverse process thickness of these ligaments around the vertebrae. Intersegmental studies indicated more flexibility of all lumbar segments in flexion than in extension [Shirazi-Adl, 1994]. This finding is further supported by studies on dolphins which revealed that all vertebral joints are stiffer in extension than in flexion [Long et al., 1997]. Furthermore, the results of lesion experiments, coupled with details of intervertebral joint anatomy,

\footnotetext{
${ }^{1}$ Because anatomical data of cheetahs are missing and cheetahs and cats are similar species, exhibiting similar pronounced spinal movements, data of a cat are used here to replace that of a cheetah.
} 
suggested that the extension resistance is ultimate due to articular joint impaction for all tested species [Gal, 1992].

However, fewer studies have successfully explained why such morphology exists in biology. One attempt suggested that this could provide a protective mechanism against intervertebral disc injury [Shirazi-Adl, 1994].

\subsection{Spine and Gaits}

The main gait investigated throughout this thesis is the bounding gait with the pronounced spinal flexion-extension movements. Bounding gait is a symmetrical gait at high speed in which the front and rear feet of each pair have equal duty factors and relative phases differing by 0.5 . Unfortunately, to date only very limited studies on the functions of spine muscles in the bounding gait are available. However, due to the similarity between the bounding and galloping gaits, we consider it reasonable to use the galloping gait as a substitution to study the functions of spine muscles. The galloping and bounding gaits share profound similarities in the properties of the spine, e.g., the posture of the spine in each phase and the way to coordinate legs [Alexander, 1982].

In addition to the galloping gait, walking and trotting gaits and their associated spinal movements will be also briefly introduced in the next section to gain a comprehensive understanding of how the spine anatomy contributes to different gaits.

\subsubsection{Functions of Spine Muscles on Gaits}

Trunk muscles can be broadly divided into epaxial muscles and hypaxial muscles. The epaxial muscles, namely vertebral extensors, develop on the dorsal side of the vertebral column and skull. These muscles are found along the entire length of the vertebral column, comprising the intrinsic muscles of the vertebral column [anatomyEXPERT, 2013a]. The hypaxial muscles form the lateral and ventral muscle walls of the trunk, and they are the neck, the thorax, the abdomen, and the pelvis [anatomyEXPERT, 2013b]. These hypaxial trunk muscles are the flexors and rotators of the vertebral column.

The function of epaxial and hypaxial muscles differ strikingly in the locomotion gaits. Table 2.1 lists their function in galloping, trotting, and walking gaits. The epaxial and hypaxial muscles play a critical role in producing sagittal movements of the body axis, which is essential for galloping gait. These sagittal movements, in addition to coordinating leg movements, are estimated to increase the top speed of a sprinting cheetah by 6 miles per hour [Hildebrand, 1959]. However, in the gaits associated with moderate speed, such as walking and trotting, their main roles are to produce lateral bending and to stabilize the trunk. 
Table 2.1: Locomotor function of epaxial and hypaxial muscles in galloping, walking, and trotting gaits [Schilling and Carrier, 2010; Fife et al., 2001].

\begin{tabular}{|l|l|l|}
\hline Gait & Epaxial muscles & Hypaxial muscles \\
\hline Galloping & Produce sagittal extension. & Flex the vertebral column. \\
\hline Trotting & $\begin{array}{l}\text { Stabilize the trunk in the sagittal plane } \\
\text { against the inertia of the CoM; } \\
\text { Assist in the production of lateral } \\
\text { bending during symmetrical gaits. }\end{array}$ & $\begin{array}{l}\text { Stabilize against vertical accelerations } \\
\text { and sagittal shearing torques; } \\
\text { Generate torsional moments; } \\
\text { Contribute to the lateral bending of the } \\
\text { body }\end{array}$ \\
\hline Walking & $\begin{array}{l}\text { Produce lateral bending; } \\
\text { Resist long-axis torsion of the trunk } \\
\text { and forces produced by extrinsic limb } \\
\text { muscles. }\end{array}$ & $\begin{array}{l}\text { Bend the trunk laterally to increase } \\
\text { stride length; } \\
\text { Stabilize the trunk against the horizot- } \\
\text { nal, lateral, and vertical components of } \\
\text { the propulsive force. }\end{array}$ \\
\hline
\end{tabular}

\subsubsection{Spinal Movements and the Associated Gaits}

Locomotion in limbed reptiles and amphibians is usually associated with lateral bending of the trunk [Daan and Belterman, 1968; Roos, 1964]. Such spinal movements have been interpreted by a mechanism of increasing stride length. According to this view, active bending of the trunk serves to move an advancing limb further forward during the recovery phase of the limb cycle [Pridmore, 1992]. Lateral bending is associated with symmetrical gaits (walking, trotting). This correlation implies that lateral bending ought to be apparent in most mammals moving at low to moderate speeds [Howell, 1944; Pridmore, 1992].

However, the evolution of mammalian locomotion is characterized by sagittal bending. This ability to dorsorventrally flex and extend the body axis enabled the evolution of asymmetrical gaits in mammals, such as galloping, half-bounding and bounding gaits. Sagittal spinal movements contribute to increasing hindlimb retraction velocity and step length, thereby enhancing animals' speed via the integration of a long aerial phase, and extended stance and swing phases [Schilling and Hackert, 2006]. Sagittal spinal movements are thought to be restricted to the lumbar part only, while the thoracic section is considered to be rigid to secure respiration during locomotion and counteract loading forces transmitted to the chest wall from limbs [Gadow, 1933].

\subsection{Inspirations Extracted from a Biological Spine}

In the previous sections, we presented the spinal engine hypothesis and biological properties of a spine (e.g., anatomy, spinal moments, the relation between the spinal movements and gaits) in vertebrate animals, which is the basis of what is used in subsequent chapters. Biorobotics suggests that drawing inspiration from nature is useful to build better technical devices. Therefore, we extracted the following important features from the above biological studies which could affect 
quadruped locomotion.

- A spine and its local musculatures are the driving force for locomotion.

- A model with two spinal joints is able to generate dynamic locomotion.

- Ligaments and muscles store and transfer energy.

- Zygapophysial joints guide and limit the spinal movements.

- Ligaments play a role in constraining the spinal movements.

- Uneven stiffness distribution of a spine has an effect on locomotion.

- There exist strong couplings between the type and range of spinal movements and the gaits.

All of these extracted inspirations will be implemented to the design of robots and tested in the following chapters. 


\section{Chapter 3}

\section{Effect of the Shape of the Spine on Spine-Driven Locomotion}

In this chapter, we examine the spinal engine hypothesis introduced in the previous chapter by taking a synthetic approach and a biorobotics methodology. Therefore, an artificial spine is designed by integrating some crucial simplified features extracted from a biological one. Then, this artificial spine is applied to the Kitty robot which has no actuation on legs. Moreover, to study how the spine morphology affects locomotion, several spine morphologies are explored which differ in the stiffness distribution and the shape of the spine.

\subsection{Development of a Biologically Inspired Spine}

Encouraged by the synthetic approach (understanding by building), we started to construct a biologically inspired spine (Fig. 3.1(c)) and then applied it to the Kitty robot in an attempt to validate this hypothesis. Table 3.1 lists the similarities in morphology and function between a biological spinal structure and its artificial counterpart.

This spine was designed in a modular architecture, so its morphology can be easily changed by the connectors. For example, the artificial vertebrae can be replaced with differently-shaped ones to form several shapes of the spinal column. Or the replacement of some silicone blocks by passive springs results in a partially actuated and partially passive morphology (Fig. 3.1(d),(e)). If we mount two rigid boards to antagonistic sides of the spine, then the degrees of freedom of such a spine will be constrained in the plane parallel to these two boards. Figure 3.1 shows all developed spinal structures, including rigid, passive, fully actuated, and partially actuated and passive spines. 
Table 3.1: Similarities in morphology and function between a biological spinal structure and its artificial counterpart.

\begin{tabular}{|l|l|l|}
\hline Biological spine & Artificial spine & Function \\
\hline Vertebra & Cross rigid segment & $\begin{array}{l}\text { Make up the spinal column; } \\
\text { Create attachment points for muscles and } \\
\text { other bones. }\end{array}$ \\
\hline Ligament & Siliconertebral disc block & $\begin{array}{l}\text { Serve as the spine's shock absorbing system; } \\
\text { Allow vertebral motion: flexion and exten- } \\
\text { sion. }\end{array}$ \\
\hline Muscle & Motor-driven string & $\begin{array}{l}\text { Connect one vertebra to another; } \\
\text { Control the range of motion of a joint. }\end{array}$ \\
\hline Zygapophysial joint & Ball joint & $\begin{array}{l}\text { Guide and limit movements of the spinal mo- } \\
\text { tion segment; } \\
\text { Protect the motion segment from anterior } \\
\text { shear forces excessive rotation and flexion. }\end{array}$ \\
\hline
\end{tabular}
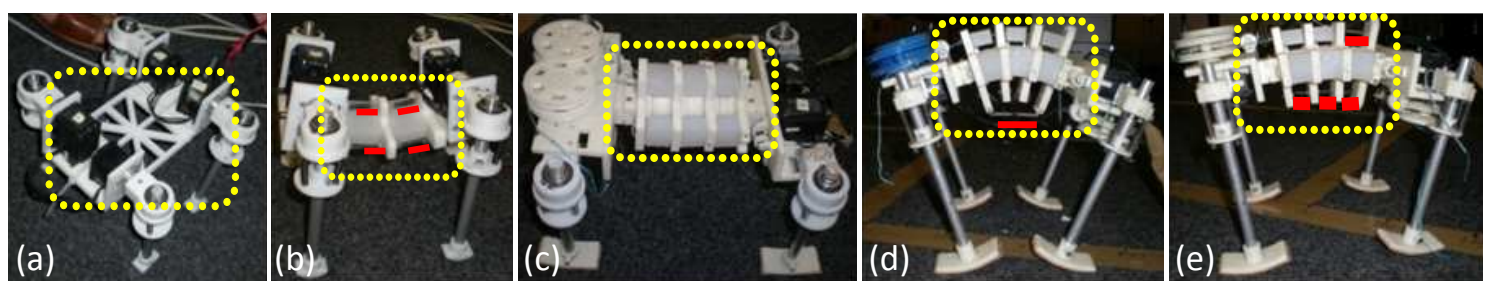

Figure 3.1: Developed spinal structures. Yellow rectangles highlight the area of the spinal structure with different morphological properties. Red lines emphasize the springs above themselves. (a) Rigid spine; (b) Fully passive spine; (c) Fully actuated spine; (d) Partially actuated spine; (e) Partially actuated spine.

\subsection{The Role of a Spine and the Effect of its Morphology on Locomotion}

In this section, we applied this highly compliant, multi-joint spine to the quadruped Kitty robot without actuation on legs. This section refers to the following publication [Zhao et al., 2011], which is enclosed as Appendix A.

Zhao, Q., Sumioka, H., and Pfeifer, R.(2011). The Effect of Morphology on the Spinal Engine Driven Locomotion in a Quadruped Robot, In the 2011 IEEE International Symposium on Adaptive Motion of Animals and Machines, pp. 51-52.

In what follows, the abstract of the paper is presented, followed by the main results related to 
the main topic of this thesis.

Abstract: The biological hypothesis of spinal engine states that the locomotion is mainly achieved by the spine, while legs only serve as assistance. Inspired by this spinal engine hypothesis, a compliant, multi$D O F$, biologically inspired spine has been developed and embedded into a quadruped robot without actuation on legs. The experimental results support this spinal engine hypothesis and reveal that this kind of robot can achieve rapid, stable, and even dynamical locomotion by appropriately tuning the spine's morphological parameters, e.g., rearranging the silicone blocks.

\subsubsection{Results}

We validated the spinal engine hypothesis and explored spine morphology regarding the stiffness distribution and the shape of the spine. The specific points addressed in this study were:

- Test the biological hypothesis of the spinal engine. A series of experiments were conducted and results showed that the Kitty robot exclusively driven by the spine is able to achieve multiple locomotion behaviors, e.g., bounding and trotting gaits, as a result of pronounced spinal flexion-extension and lateral movements, respectively. Turning behavior can also be realized by the combination of the bounding and trotting gaits. This supports the spinal engine hypothesis which states that the spine plays an essential role in vertebrate locomotion.

- Effect of the stiffness distribution and the shape of a spine. To better understand the correlation between a spine's morphological property and locomotion behavior, several experiments were conducted under the condition of spinal structures which differ in the stiffness and the shape distribution. Preliminary experimental results showed that the rapid and stable forward motion can be achieved when the silicone blocks are distributed evenly in the spine, whereas more dynamical movements can be observed by taking out some silicone blocks. This phenomenon was observed in the cases of both the rectangle and rhombus morphologies, but the robot equipped with a rhombus-shaped spine can lift up the rear legs, as a result of the upward force transmitted from such a spine.

\subsubsection{Contributions}

This study investigated the possibility of a spine to be an engine to drive locomotion from a mechanical point of view and how the stiffness distribution and the shape of a spine affect locomotion. The main contributions are as follows:

- It examined the concept of the spinal engine by manufacturing a biologically inspired spine and embedding it to a physical quadruped robot. It showed the merits of understanding by building. 
- It explored the effect of spine morphology on locomotion and revealed that a robot can achieve rapid, stable, and even dynamical locomotion by appropriately tuning the spine's morphological parameters, e.g., rearranging the silicone blocks. 


\section{Chapter 4}

\section{Embodiment Enables
Spine-Driven Locomotion}

This chapter is a summary of the following publication [Zhao et al., 2012], which is enclosed as Appendix B.

Zhao, Q., Nakajima, K., Sumioka, H., Yu, X., and Pfeifer, R.(2012). Embodiment Enables the Spinal Engine in Quadruped Robot Locomotion, In the 2012 IEEE/RSJ International Conference on Intelligent Robots and Systems (IROS), pp. 2449-2456.

In what follows, the abstract of the paper is presented, followed by the main results related to the main topic of this thesis.

Abstract: The biological hypothesis of spinal engine states that locomotion is mainly achieved by the spine, while the legs may serve as assistance. Inspired by this hypothesis, a compliant, multiple degreeof-freedom, biologically inspired spine has been embedded into a quadruped robot, named Kitty, which has no actuation on the legs. In this paper, we demonstrate how versatile behaviors (bounding, trotting, and turning) can be generated exclusively by the spine's movements through dynamical interaction between the controller, the body, and the environment, known as embodiment. Moreover, we introduce information theoretic approach to quantitatively study the spine internal dynamics and its effect on the bounding gait based on three spinal morphologies. These three morphologies differ in the position of a virtual spinal joint where the spine is easier to get bent. The experimental results reveal that locomotion can be enhanced by using the spine featuring a rear virtual spinal joint, which offers more freedom for the rear legs to move forward. In addition, the information theoretic analysis shows that, according to the morphological differences of the spine, the information structure changes. The relationship between the observed behavior of the robot and the corresponding information structure is discussed in detail. 


\subsection{Results}

In this study, we demonstrated the spine-driven locomotion generated through embodiment in the Kitty robot. The Kitty robot features a biologically inspired spine and is exclusively driven by this spine. Its legs are fixed to the body and therefore have no relative rotation with respect to the spine. This study addressed the following specific points:

- Embodiment enables spine-driven locomotion in the Kitty robot. We demonstrated how embodiment enables versatile spine-driven locomotion (e.g., bounding gait, trotting gait, and turning behavior) in the Kitty robot. These behaviours are generated through the embodied coupling between the controller, the body, and the environment. More precisely, they can be observed only when the Kitty robot interacts with the environment. The real importance of embodiment comes from the interaction between physical processes, i.e., information processes.

- How the position of a virtual spinal joint and the stiffness distribution of the spine affect spine-driven locomotion? Spinal morphologies differing in the virtual spinal joint (VSJ) were explored on the bounding and trotting gaits. A virtual spinal joint is defined as a simplified rotational joint of the spine, where this spine is easier to get bent due to the lack of silicone block. These spinal morphologies are named spine morphology 1 (SM1), spine morphology 2 (SM2), and spine morphology 3 (SM3) with a middle, front and rear virtual spinal joint, respectively. It was demonstrated that the speed of the robot with SM3 or SM2 is greater than the one with SM1 due to the freedom offered by the absence of some silicone blocks in the spine. We concluded that the robot's performance is better if the stiffness in the downside of the spine is lower than the one in the upside. In addition, we observed that SM3 exhibits the best performance in these two gaits in terms of speed, because a rear VSJ helps rear part of the body to gain more freedom to bend, pull the rear legs forward, thus leading to a longer stride. This is in agreement with biological finding that lumbar spine locating in the rear side of the spine is the largest of the movable vertebrae [Taylor, 2012].

- How the embodied coupling induced by the change of spine morphology can be quantified by information theory.

By using an information theoretic analysis, we characterized the information structure of the sensorimotor variables in the bounding gait with three spinal morphologies. The results suggested that through the embodied interaction with the environment, the specific body morphology is crucial in shaping the resulting information structure. It was observed that information transfers and the associations between the variables brought about by the interaction with the environment tend to increase according to the change of spine morphology. This enhancement of the information structure seems to have a correspondence with the change of velocity. 


\subsection{Contributions}

This study mainly investigated how embodiment enables spine-driven locomotion and how to quantify the information structure induced by the morphology. The main contributions are as follows:

- Versatile spine-driven behaviors were demonstrated through the embodied coupling between the controller, the body, and the environment.

- The information theoretical measures were tested and then proved to be an effective tool to quantify the information structure in sensorimotor interactions induced by the morphology. This information structure can be used to explain the behavior of a robot.

- The effect of position of a virtual spinal joint on locomotion was investigated, and the results are in agreement with biological findings. 



\section{Chapter 5}

\section{Effect of the Number of Spinal Joints on Spine-Driven Locomotion}

This chapter is a summary of the following publication [Zhao et al., 2014], which is enclosed as Appendix C.

Zhao, Q., Sumioka, H., Nakajima, K., Yu, X., and Pfeifer, R.(2014). Spine as an Engine: Effect of Spine Morphology on Spine-Driven Quadruped Locomotion, Advanced Robotics, 28(6): pp. 367378.

In what follows, the abstract of the paper is presented, followed by the main results related to the main topic of this thesis.

Abstract: In quadruped animals, spinal movements contribute to locomotion in terms of controlling body posture and integrating limb and trunk actions. In this paper, we develop quadruped models with different numbers of spinal joints to demonstrate the spine-driven locomotion. Actuated spinal joint(s) are exclusively employed to these models with a minimalistic control strategy. We choose some typical individuals from two models and analyze them on gait properties. Results show that employing the spine morphology with two joints can greatly enhance the stability and speed of locomotion by readjusting center of mass, increasing the stride length, and generating double flight phases similar to running cheetahs' gait, which makes a significant difference in the speed and the gait. Furthermore, we explore and compare models with more spinal joints. Results show that all gaits emerged from them can be categorized into three types (bounding, bounding with double flight phase, and stotting (pronking) gaits). Overall, bounding gait with double flight phases is a more biologically inspired locomotive behavior; model with two spinal joints could be sufficient to emulate biological spine-driven locomotive behaviors. 


\subsection{Results}

In this study, we investigated the effect of the number of the spinal joints on spine-driven locomotion in simulation. The simulated models were implemented in Mathworks matlab 2009, together with the SimMechanics toolbox, which provides a multibody simulation environment. This study addressed the following specific points:

- Development of a simulation model with a minimalistic control strategy. Two models differing in the number of spinal joints were developed to demonstrate the spine-driven locomotion behavior. One model called $M 1$ has one spinal lumbosacral joint; the other one named $M 2$ has lumbosacral joint (LJ) and thoracic joint together (TJ). In these models, the fore and rear legs are fixed to the ends of the spine and have no relative rotation with respect to it. Such a design is straightforward to study the function of a spine and the effect of spine morphology on locomotion. For the same reason, a minimalistic control strategy was employed to this model, in which the angular position of the spinal joints is determined by the sinusoidal curve.

- Overall exploration based on two spinal morphologies. Experiments were systematically conducted in models $M 1$ and $M 2$. Simulation results showed that there exist three gaits in $M 2$ with two spinal joints: bounding, bounding with double flight phases (BDFP), and stotting gaits. However $M 1$ with one spinal joint is only able to generate bounding gait. Overall speaking, $M 2$ performs better than $M 1$ in terms of the speed and stability due to two factors. First, $M 2$ is able to produce more freedom to pull the rear legs forward, increase the stride length, and move the CoM more efficiently forward, thus increasing the speed. Second, it benefits stability by using additional TJ to readjust unstable posture or enhance the flexion-extension movements generated by the LJ.

- Dynamic locomotion induced by double flight phases. To investigate how the BDFP gait is generated and how it contributes to the locomotion, we selected the fastest individual in BDFP gait from $M 2$, namely $I 3$, and compared it with $I 1$ and $I 2$, which are the fastest ones in bounding gait in models $M 1$ and $M 2$, respectively, and analyzed them on gait properties. It was observed that $I 3$ can greatly enhance the speed of locomotion mainly by generating double flight phases similar to a running cheetah's gait, which makes a significant difference in the speed and the gait, compared to I1 and I2. I3 reaches the maximal extension and flexion in two flight phases per stride, while $I 1$ and $I 2$ are only suspended once in each stride. Moreover, the gait of $I 3$ exhibits a greater proportion of flight in total stride. These results are in agreement with studies of the motions of the running cheetah and horse [Hildebrand, 1959]. A horse, with relatively rigid spine generating less spinal movements [Townsend et al., 1983], can be represented by I2, and a cheetah, featuring pronounced spinal movements, is suitable to be simplified as I3. We concluded that the 
double flight phases, together with the greater proportion of flight, contribute to its longer stride [Hildebrand, 1959].

- Stotting gait emerged from pronounced spinal flexion-extension movements. Stotting gait in which the model's four legs jump off and on the ground at the same time emerges when the spinal flexion-extension is pronounced enough. It exhibits much higher ground clearance and wider vertical excursion of center of mass. We found that the stance phase between spinal flexion and extension phase is missing, which has the function to transfer energy and power the spinal extension movements [Alexander, 1988]. The absence of such a phase is considered to lead to energy inefficiency and slow speed. Therefore, stotting gait is not suitable for fast locomotion. Instead, it plays a role in communication [Caro, 1986], e.g., to tell the predator that it has been seen, and warn its group members of the danger.

- Exploration on multiple spinal joints. To check if this simplified model $M 2$ is sufficient to emulate biological spine-driven locomotion, we extended the experiments by introducing more spinal joints, while keeping most of the settings the same.

Interestingly, it was observed that all of the successful individuals over all spine morphologies can be categorized into three types: bounding gait, BDFP gait, and stotting gait, . The same categorization of the gaits is also observed in $M 2$. Generally speaking, BDFP gait outperforms the other two gaits regarding speed. Moreover, BDFP spreads almost over all of the morphologies, except for $M 1$. Therefore, these two aspects indicate that BDFP gait is more biologically plausible for fast locomotion, compared to stotting and bounding gaits. Since $M 2$ with two spinal joints is able to produce such BDFP gait, we believe that $M 2$ is sufficient to emulate spine-driven locomotion. This question arises: if two joints are sufficient, why animals evolved many more joints? One possible answer is that with more spinal joints, it is easier to form a smoother curvature of the spine in an attempt to distribute the load along the spine, thus protecting the spine from being damaged [ $\mathrm{Pal}$ and Sherk, 1988; Han et al., 2013].

\subsection{Contributions}

This study investigated the optimal number of spinal joints for spine-driven locomotion. The main contributions are as follows:

- Quadruped models with different numbers of spinal joints were developed to demonstrate the spine-driven locomotion.

- Bounding gait with double flight phases was tested to be a more biologically plausible gait to achieve fast locomotion, compared to stotting and bounding gaits. 
- A model with two spinal joints was proposed and shown to be sufficient to emulate biological spine-driven locomotion behavior regarding gait properties, such as gait pattern, ground clearance, attack angle, etc. 


\section{Chapter 6}

\section{Flexible Spine as a Computational Resource}

This chapter is a summary of the following publication [Zhao et al., 2013b], which is enclosed as Appendix D.

Zhao, Q., Nakajima, K., Sumioka, H., Hauser H., and Pfeifer, R.(2013). Spine Dynamics as a Computational Resource in Spine-Driven Quadruped Locomotion, In the 2013 IEEE/RSJ International Conference on Intelligent Robots and Systems (IROS), pp. 1445-1451.

In what follows, the abstract of the paper is presented, followed by the main results related to the main topic of this thesis.

Abstract: Recent results suggest that compliance and nonlinearity in physical bodies of soft robots may not be disadvantageous properties with respect to control, but rather of advantage. In the context of morphological computation one could see such complex structures as potential computational resources. In this study, we implement and exploit this view point in a spine-driven quadruped robot called Kitty by using its flexible spine as a computational resource. The spine is an actuated multi-joint structure consisting of a sequence of soft silicone blocks. Its complex dynamics are captured by a set of force sensors and used to construct a closed-loop to drive the motor commands. We use simple static, linear readout weights to combine the sensor values to generate multiple gait patterns (bounding, trotting, turning behavior). In addition, we demonstrate the robustness of the setup by applying strong external perturbations in form of additional loads. The system is able to fully recover to its nominal gait patterns (which are encoded in the linear readout weights) after the perturbation has vanished. 


\subsection{Results}

Previous studies (chapters 3, 4,5) mainly tested the spinal engine hypothesis and demonstrated that the spine is a main component to drive the locomotion from a mechanical point of view. This study investigated the possibility of the flexible spine in the Kitty robot to be a computational resource in the context of morphological computation and addressed the following specific points.

- A compliant spine as a computational device. The theory of morphological computation states that a complex physical body of soft robots could be a potential computational resource, due to its elasticity and nonlinearity inherently [Hauser et al., 2011]. This theory was then exploited and implemented to a spine-driven robot featuring a compliant spine. It was demonstrated that such a spine can serve as a computational resource to generate different behaviors, such as bounding gait, trotting gait, and turning behavior. The results revealed that this computational resource (compliant spine) together with linear and static readouts and feedback loops is able to encode movement patterns, produce rhythmic patterns, and learn new patterns.

- Multiple behaviors generated by the same physical body. Experimental results indicated that multiple behaviors (bounding gait, trotting gait, and turning behavior) can be produced by the same fixed physical body, simply by readjusting the weights of the linear readouts. This shows the computational power of such a compliant spine.

- Robustness of such a system against external perturbation. The robustness of the learned behavior was demonstrated by applying additional load as external perturbations. It was shown that this system is able to fully recover to its nominal gait patterns (which are encoded in the linear weights) after the perturbation has vanished, even though the load is increased to two-thirds of the Kitty robot's own weight.

\subsection{Contributions}

We investigated the potential to use a compliant body as a computational resource within the framework of morphological computation. The main contributions are as follows:

- We implemented and exploited morphological computation in a spine-driven quadruped robot by using its flexible spine as a computational resource.

- We explored the morphology of the sensors to detect body dynamics of a compliant spine. The spine's complex dynamics are captured by 32 randomly embedded force sensors and used to construct a closed-loop to drive the motor commands.

- We tested the robustness of such a system by applying additional load as external perturbations in bounding gait. 


\section{Chapter 7}

\section{Effect of Spine Actuation and Stiffness on Locomotion}

This chapter is a summary of the following publication [Zhao et al., 2013a], which is enclosed as Appendix E.

Zhao, Q., Ellenberger, B., Sumioka, H., Sandy, T., and Pfeifer, R.(2013). The Effect of Spine Actuation and Stiffness on a Pneumatically-driven Quadruped Robot for Cheetah-like Locomotion, In the 2013 IEEE International Conference on Robotics and Biomimetics (ROBIO), pp. 1807-1812.

In what follows, the abstract of the paper is presented, followed by the main results related to the main topic of this thesis.

Abstract: Biological research has concluded that the actuation of the spine contributes significantly to the performance of quadrupeds in terms of controlling body posture, and integrating limbs and trunk actions. Inspired by this biological finding, we develop a pneumatically-driven quadruped robot called Renny with configurable spine morphology to study how the spine contributes to cheetah-like running. Three spine morphologies: rigid spine, passive spine, and actuated spine, are introduced and tested in the Renny robot. In addition, we investigate the effect of the stiffness distribution of the spine muscles in the passive case. The experimental results show that the passive one where the dorsal stiffness is higher than the ventral stiffness can run faster, even faster than in jthe rigid case. Moreover, the coordination between the leg and the actuated spine is studied in actuated spine morphology. We found that when the spinal movements are synchronized with the leg movements, the speed is much faster. In the actuated case, both flexion and extension benefit the increase of the speed by advancing limbs rapidly and increasing the limb swing. 


\subsection{Results}

In this study, we investigated the effect of spine actuation and its stiffness distribution on locomotion, and the way to coordinate the spine and the leg movements in the Renny robot. Renny is a pneumatically-driven quadruped robot with configurable spine morphology. It features spine actuation, as well as hip and knee joint actuation. This study addressed the following specific points:

- Basic properties of pneumatic artificial muscles. Experiments were first conducted to understand the basic features of McKibben muscles, i.e., how varying the supplied air pressure and preload affects muscle contraction. It was observed that there exists the same tendency between contraction and preload for each pressure. The maximal contraction increases as the preload is increased up to a threshold where the actuator is nearly fully stretched. Beyond this threshold, the contraction decreases. Last, hip and spine muscles with higher ratio of the outer sleeve to the inner tube exhibit the higher contraction capability, compared to knee muscles with lower ratio.

- Passive spine with suitable stiffness. Quantitative studies revealed that if the supplied air pressure of the Renny robot's upper spine muscles is higher than the lower ones, then Renny's speed is even faster. In other words, relatively higher stiffness in the upside of the spine is beneficial to locomotion, which is in agreement with biological studies [Long, 1997].

- Comparison between a passive spine and a rigid spine We noticed that Renny with a passive spine with appropriate stiffness can run faster than the best rigid case. This might be explained by the soft and compliant characteristics of PAMs. One of the similarities between PAMs and biological muscles is compliance, which contributes to locomotion by working like a spring transferring energy between the front and rear body in bounding gait [Hildebrand, 1959].

- Coordination between the spine and legs. In the actuated spine morphology, we simplified the spinal movements in such a way that when the front legs flex, the spine extends, and when the rear legs flex, the spine flexes. This simplification is reasonable, because it is more likely to increase the forward flexion of the limbs and advance the limbs more rapidly, as suggested by biological findings [Hildebrand, 1959]. Therefore, two parameters which are phase delay between the shoulder flexor and the spine extensor ( $\left.\Psi_{\text {ShoulderFlex,SpineExte }}\right)$, and phase delay between the hip flexor and the spine flexor $\left(\Psi_{\text {HipFlex,SpineFlex }}\right)$ are employed to control the spinal movements and coordinate leg movements. The results showed that there exists a strong coupling between the spine's extension and the front legs' flexion. The shorter the resultant timing $\left(\Psi_{\text {ShoulderFlex,SpineExte }}\right)$ is, the faster the robot can run. More interestingly, the best individual is found when these two parameters equal zero. This 
revealed that the synchronization between the spine and leg movements is crucial to increase the speed.

\subsection{Contributions}

This study first examined the effect of actuation type and the stiffness distribution on locomotion. Moreover, the strategy to coordinate the spine and the legs were investigated. The main contributions are as follows:

- A pneumatically-driven quadruped robot was developed in a modular architecture to mimic cheetah-like running. To achieve this goal, a spine with configurable morphology is designed in such a way that it can easily change to be rigid, passive, or actuated.

- Some design principles were extracted, e.g., type of actuation, stiffness distribution, which might provide insights for other robots to enhance locomotion.

- A control strategy with two introduced parameters was proposed to coordinate the movements between a spine and legs. 



\section{Chapter 8}

\section{Discussion and Conclusion}

In the preceding chapters, we have investigated the potential of a spine to be an engine for locomotion and its morphological effect on locomotion mainly by taking a synthetic approach. In what follows, we summarize the main results and discuss their implications for robotics and biology.

This chapter is structured as follows: we recall the main results from the individual publications presented in chapters 3 to 7 , followed by implications for biology and robotics. Then we discuss future directions of our work. At the end, final conclusions are drawn.

\subsection{Summary of Results}

This section summarizes the results to address the research questions and hypotheses proposed in the introduction.

\section{Research Question 1: Whether a spine is possible to be an engine for vertebrate locomotion?}

In Chapters 3, 4, and 5, we demonstrated the spine-driven locomotion in the Kitty robot and the simulated models. These robots are designed in such a way that only the spine is actuated while the legs are fixed to the body. This design is simple and straightforward to demonstrate the spine-driven locomotion. Kitty can exhibit different behaviors, such as bounding gait produced by sagittal spinal movements, trotting gait generated by lateral spinal movements, and turning behavior caused by the combination of sagittal and lateral spinal movements (Chapters 3, 4). A simulated model with two spinal joints which represent lumbosacral joint and thoracic joint can achieve more dynamic gaits, compared to the behaviors observed in the Kitty robot featuring one virtual spinal joint (Chapter 5). This model exhibits bounding gait, bounding with double flight phases (BDFP), and stotting gait.

In short, these studies suggested that a spine is possible to be an engine for vertebrate locomotion and the spine engine hypothesis is applicable not only to limbless animals (e.g., snake), but 
also to legged vertebrate animals.

Research Question 2: What are the key factors of a biological spine to affect quadruped locomotion?

In Chapter 2, we presented the function, the biological anatomy, the movements of a spine and its associated gaits in vertebrate animals, which serve as the basis and starting point of further investigation. From the literature, we extracted some important features affecting quadruped locomotion. For example, ligaments and muscles store and transfer energy; uneven stiffness distribution of a spine has an effect on locomotion; ligaments play a role in constraining the spinal movements. Moreover, studies on certain sections of the spine suggest a way to simplify the number of spinal joints and determine their positions. Additionally, the relation between the type and range of spinal movements and the gaits helps us to study the underlying mechanism of how a spine could be a main driving component for locomotion. All of these features have been extracted and implemented to the design of the robots.

\section{Research Question 3: How the spine morphology affects spine-driven locomotion?}

This question was tackled by extracting ideas from a biological spine, implementing them to an artificial spine, and then testing them in robotic platforms.

Hypothesis 3.1: The shape of a spinal column has an effect on locomotion.

A series of experiments were conducted in the Kitty robot to study the effect of the shape of the spinal column on locomotion (Chapter 3). Two types of the spinal column were employed and tested: one is a rhombus-shaped spine, which is closer to a biological spine; the other one is a rectangle-shaped spine, which is a much more simplified model. Preliminary experimental results showed that the rapid and stable forward motion can be achieved by the robot with a rectangleshaped spine, but more dynamic behaviors can be observed in the robot with a rhombus-shaped spine. In the former case, Kitty hardly exhibits ground clearance for any leg, while in the latter case, it can lift up the rear legs, because the transmitted force can be decomposed into two directions: one upward and one horizontal. The upward force can lift up the rear legs. However, the design of this rhombus-shaped spine results in high friction for strings passing through it, because of the vertex in the middle of the spine.

Hypothesis 3.2: A model with one actuated spinal joint is possible to generate bounding, trotting gaits and turning behavior.

The Kitty robot is endowed with a biologically inspired spine with one virtual spinal joint (VSJ) in which the spine is more likely to get bent. It was demonstrated that the robot with such a virtual spinal joint is able to generate versatile behaviors (bounding gait, trotting gait, and turning behavior) through the embodied coupling between the controller, the body and the environment (Chapters 3, 4).

Hypothesis 3.3: Rear position of a spinal joint benefits locomotion by providing the rear legs with more freedom to swing further. 
Three different spinal morphological configurations differing in the position of VSJ were explored on the bounding and trotting gaits in the Kitty robot (Chapter 4). Experimental results showed that the speed of the morphology with front VSJ (SM3) and the morphology with rear VSJ (SM2) is greater than of the morphology with middle VSJ (SM1) due to the freedom offered by the absence of some silicone blocks in the spine. In addition, we observed that Kitty with SM3 performs best in these two gaits regarding speed, because a rear VSJ helps the rear part of the body to gain more freedom to bend, pull the rear legs forward, thus leading to a longer stride. This is in agreement with the biological finding that lumbar spine locating in the rear side of the spine is the largest of the movable vertebrae [Taylor, 2012].

Hypothesis 3.4: A robot with a spine of which the dorsal stiffness is higher than the ventral stiffness runs faster than the one with other configurations.

It was observed that the Kitty robot with SM3 or SM2 moves faster than the one with SM1, because the freedom offered by the absence of some silicone blocks in the downside of the spine (Chapter 4). This also suggested that a robot with a spine of which the dorsal stiffness higher than the ventral stiffness runs faster, which is in agreement with biological findings [Long, 1997]. This finding was further supported by systematic experiments on a pneumatically-driven quadruped robot called Renny (Chapter 7). This study revealed that higher stiffness in the upside of the spine than that in the downside is beneficial to locomotion.

Hypothesis 3.5: A model with two spinal joints is sufficient to generate more dynamic behaviors.

The effect of the number of spinal joints was investigated in simulated models (Chapter 5). We found that there are three gaits (bounding gait, bounding gait with double flight phases (BDFP), and stotting gait) in $M 2$ with two spinal joints, while only bounding gait is observed in $M 1$ with one spinal joint. Overall speaking, $M 2$ outperforms $M 1$ in terms of the speed and stability. $M 2$ allows for more freedom to pull the rear legs forward, thus increasing the stride length. Additionally, it benefits stability by using additional $\mathrm{TJ}$ to readjust unstable posture or enhance the flexion-extension movements generated by the LJ. Among the observed three gaits in $M 2$, BDFP is the fastest gait largely due to double flight phases and a great proportion of flight in total stride similar to a running cheetah's gait, which makes a significant difference in the speed and the gait.

Experiments were extended by introducing more spinal joints to check if this simplified model $M 2$ is sufficient to emulate biological spine-driven locomotion. Interestingly, we found that all of the gaits over all spine morphologies can be categorized into these three gaits (bounding, BDFP, and stotting gaits). The same categorization of the gaits is also observed in $M 2$. Generally speaking, BDFP gait is faster than the other two gaits. Moreover, BDFP can be found in almost over all morphologies, except for $M 1$. These two aspects indicated that BDFP gait is more biologically plausible for fast locomotion. Therefore, it was concluded that $M 2$ is sufficient to emulate dynamic spine-driven locomotion, because it is the simplest model to produce such BDFP gait.

Hypothesis 3.6: A spine with compliant elements stores and transfers energy.

We observed that the Renny robot with a passive spine configured with appropriate stiffness 
can run faster than the best rigid case (Chapter 7). This might be caused by the soft and compliant characteristics of McKibben muscles, which are similar to biological muscles. Such features enhance locomotion by working like a spring transferring energy between the front and rear body in bounding gait [Hildebrand, 1959].

Research Question 4: Is it possible to use a compliant spine as a computational resource to drive locomotion?

The Kitty robot features a biologically inspired spine which is an actuated multi-joint structure consisting of a sequence of soft silicone blocks. Such a spine was employed to test the possibility to be a computational resource in the context of morphological computation (Chapter 6). Its complex dynamics captured by a set of force sensors are used to construct a closed-loop to generate the commands to the motors.

The task of Kitty is to generate locomotion behaviors by using the dynamics of its spine captured by randomly distributed force sensors. Results showed that after the teaching and learning phases, this computational resource (compliant spine) together with linear and static readouts and feedback loops can encode movement patterns and produce rhythmic locomotion patterns. We observed that with the same physical spine and the same arrangement of the sensors, such a system can learn multiple locomotion patterns (bounding gait, trotting gait, and turning behavior) simply by readjusting the weights of the linear readouts. Moreover, the robustness of the setup was tested by applying strong external perturbations in the form of additional loads in the front of the Kitty robot. It was observed that after the perturbation has vanished, the ability of emulating desired signal (i.e., signals sent to the motors to drive locomotion) of such a system is restored and locomotion pattern continues, even when the load is increased to two-thirds of this robot's own weight.

\section{Research Question 5: How to coordinate the movements of a spine and the legs?}

The Renny robot features spine actuation, as well as hip joint and knee joint actuation (Chapter 7). We simplified its spinal movements in such a way that when the front legs flex, the spine extends, and when the rear legs flex, the spine flexes. This simplification is reasonable, because it is more likely to increase the forward flexion of the limbs and advance the limbs more rapidly, as suggested by biological findings [Hildebrand, 1959]. Therefore, two parameters which are phase delay between the shoulder flexor and the spine extensor $\left(\Psi_{\text {ShoulderFlex,SpineExte }}\right)$, and phase delay between the hip flexor and the spine flexor $\left(\Psi_{\text {HipFlex,SpineFlex }}\right)$ were employed to control the spinal movements and coordinate the leg movements.

We observed that it is more likely for Renny to reach a faster speed when $\Psi_{\text {Shoulder Flex,SpineExte }}$ is greater than $-12.5 \%$ and less than $25 \%$. It suggested that there is a strong coupling between the spine's extension and the front legs' flexion. The shorter the resultant timing $\left(\Psi_{\text {ShoulderFlex,SpineExte }}\right)$, the faster the robot can run. More interestingly, the best individual is found when both $\Psi_{\text {HipFlex,SpineFlex }}$ and $\Psi_{\text {ShoulderFlex,SpineExte }}$ equal zero. This revealed that the synchronization between the spine 
and leg movements is crucial to increase the speed.

\subsection{General Implications}

Our research on the investigation of the locomotor function of a spine and its morphological effect also provides some implications for biology and robotics.

\subsubsection{Implications for the Spinal Engine}

From a mechanical point of view, how locomotion is achieved exclusively by employing spine actuation was demonstrated in Chapters 3-5. The experimental results showed that the Kitty robot and the simulated models can generate versatile behaviors (e.g., bounding gait, trotting gait, turning behavior, stotting gait, etc.) through the interaction with the environment, as a result of pronounced spinal movements.

In addition to connecting the front and rear parts of the body, the developed compliant spine in Kitty serves as a computational resource to generate different behaviors in the context of morphological computation (Chapter 6). Such a compliant spine together with linear and static readouts and feedback loops is able to encode movement patterns, produce rhythmic patterns, and learn new patterns.

It was reported that locomotor central pattern generators (CPGs) are capable of producing coordinated patterns of high-dimensional rhythmic output signals, such as walking, running, and swimming, while receiving only simple, low-dimensional, input signals [Ijspeert, 2008]. Some features of CPG, such as high nonlinearity, rich dynamics, powerful modulation capacity by sensory input, and oscillatory behaviors, are considered to be important to perform real-time computation. These features are also shared in reservoir computing [Maass et al., 2002; Jaeger and Haas, 2004]. This implies that reservoir computing could be considered analogous to CPGs.

Morphological computation is one of the physical versions of reservoir computing. In other words, the concept of reservoir computing is applied to a random network of mass-spring systems. Instead of using a neural network or a network of leaky integrators (as in standard approaches for reservoir computing), MC models employ a compliant physical body as a reservoir. Experimental results revealed that the spine together with linear and static readouts and feedback loops can be used to generate multiple locomotion behaviors simply by readjusting the weights of the linear readouts. The function of such a system seems to be comparable to CPGs which can generate various rhythmic moments. Therefore, this suggests that a compliant spine can serve as a computational resource, which implicitly supports the hypothesis of the spinal engine from a controlling perspective. 


\subsubsection{Implications for a Biological Spine}

The main motivation for robotics researchers to study natural systems is that these systems have been optimized for specific tasks and environments by evolution. However, why such systems evolve to the current form still remains an open question. The relation between morphologies and specific tasks can be elucidated by using the synthetic methodology, i.e., understanding by building. Therefore, in this thesis, several platforms in simulation and physical world were developed to learn such a relation by evaluating biological hypotheses. Results of our studies on robotics platform are consistent with biological hypotheses to a large extent, thus confirming the functions of these morphologies. For example, it was observed in Kitty that a rear virtual spinal joint is beneficial to bend rear body part further, pull the rear legs more forward, thus leading to a longer stride and faster speed, compared to other locations (Chapter 4). This is in agreement with the biological finding that lumbar spine located in the rear side of the spine is the largest of the movable vertebrae [Taylor, 2012]. Another example is the stiffness distribution of the spine. More specifically, results in Renny revealed that a spine of which the dorsal stiffness higher than the ventral stiffness is beneficial to increase locomotion speed (Chapter 7), which is also consistent with biological studies [Long, 1997].

\subsubsection{Implications for Robots with a Rigid Spine}

Biological findings and all results in this thesis suggested that the spine may play a dominant role in vertebrate locomotion and the spine morphology has a great influence on locomotion performance. For example, the position of a virtual spinal joint, the number of spinal joints, and the stiffness distribution of spine muscles are such crucial parameters. Their influences and contributions on locomotion have been explicitly illustrated in previous chapters. In summary, our studies tested the locomotor function of a spine on vertebrate locomotion, which would encourage robotics researchers to pay much attention to the effect of a spine when designing a robot. Moreover, this thesis also provides design principles (e.g., the position or the number of spinal joints, the stiffness distribution of the spine) to optimize the spine morphology, and thus improves performance regarding speed, maneuverability, stability, and energy efficiency.

\subsection{Directions for Future Research}

Since this study is exploratory and experimental, it can be continued in many directions. First, the physical platforms (e.g., the Renny robot) should be technically improved to get closer to the morphology of a cheetah. Based on these improved platforms, more features of the spine can be investigated, for example, the relation between the spine actuation type and the energy efficiency. Second, in the context of morphological computation, more asymmetrical spine features can be explored to increase the accuracy of the spine reservoir in the Kitty robot. Moreover, the 
combination between morphological computation and conventional reservoir computing can be investigated to understand how the body and brain cooperate to achieve gait switching.

\subsubsection{Improvement of the Renny Robot}

Renny is a robot driven by McKibben artificial muscles, so it needs to be provided with an efficient, lightweight and properly working pneumatic system, including tubes, valves, and air compressor (Chapter 7). As a first step, our main focus is to test the function of the spine and how to coordinate the spine with legs. Therefore, we did not put too much effort in optimizing the pneumatic system. All of the valves and the air compressor are mounted outside the robot, and the robot is tethered by tubes. Such a configuration affects the behavior to some extent. For example, when we are holding the tubes, it is more likely to change the position of center of mass or misguide the locomotion direction. In the future, we will improve the hardware by mounting much smaller valves and air compressor into the robot to make it self-contained as much as possible. Furthermore, more complex spine morphologies (more vertebrae and muscles), and movements (twisting, lateral bending, or the combination) will be studied and implemented to this robot, to further examine the role and the effect of the spine on quadruped locomotion.

\subsubsection{Energy Efficiency of the Renny Robot}

It was observed that Renny with a passive spine can run faster than the one with a rigid one if the passive stiffness is properly set, but Renny with an actuated spine is the fastest one (Chapter 7). On one hand, such an additional spine actuation joint provides more energy to extend and flex the spine, thus increasing the stride length, but on the other hand, it also consumes more energy, compared to the passive spine. Therefore, it is of crucial importance to propose a method to measure which configuration is more energy efficient. So far, a widely used method in robots driven by electric motors is specific resistance, which is a dimensionless quantity, calculated as $\frac{\text { average power expenditure }}{\text { weight } \cdot \text { gravitational acceleration } \cdot \text { velocity }}$ [Gabrielli and von Karman, 1951].

Similar method called specific energetic cost of transport $c_{e t}=\frac{\text { energy used }}{\text { weight } \text { distance traveled }}$ was proposed in [Collins et al., 2005]. A related measure is mechanical energy effectiveness $c_{m t}$ which assigns an energy cost only to the mechanical work of the actuators and not to the chemical or electrical energy used. Mechanical effectiveness $c_{m t}$ isolates the effectiveness of the mechanical design and control-system aspects from the motor/muscle efficiency. Such a method is also applicable to pneumatically-driven robots. One example is the Delft biped robot which is based on net positive mechanical work at the joints by measuring the torques at these joints as a function of angle and time [Collins et al., 2005]. Our focus lies on the role of the spine and its morphology effect on locomotion, so we only take into account the mechanical work of the pneumatic actuators, instead of the used eclectic energy (e.g., power consumption of air compressor and electric circuit boards). 
To determine the torque of joints in Delft, it is required to measure the force-length relation of the McKibben muscles at the operating pressure first [Collins et al., 2005]. So far there have been few attempts to develop such kind of sensors for McKibben muscles. For example, Kuriyama et al. proposed a method of estimating the length of the actuator from the circumferential displacement, which can be measured by a sensor made of electro conductive, flexible rubber [Kuriyama et al., 2009]. The sensor error occurs as a result of the geometrical shape of the actuator, because the actuator has a triangle shape at the two ends and a rectangle shape at the middle part. Moreover, the shape of the actuator decreases the accuracy of the interweave angle of the braided mesh sleeve. Wakimoto et al. developed a soft displacement sensor and incorporated it in the Mckibben actuator [Wakimoto et al., 2005]. This compliant sensor is capable of reducing the system size because it is incorporated in the actuator. However, in practice, such feature increases the difficulty to remount the sensor in the handmade McKibben muscle once the muscle gets replaced. In short, the requirements on smaller size, light weight, higher accuracy, more flexible material, and easy installation pose challenges to develop the right sensors for McKibben muscles. Thus, it will be one of our future directions.

\subsubsection{Energy Transfer and Store by a Spine}

It was observed that Renny with a passive spine of which dorsal stiffness is higher than ventral stiffness runs faster than the robot with a rigid spine (Chapter 7). We attribute this feature to the compliance of the spine, which might play a role as a nonlinear spring to enhance locomotion by transferring and storing energy. As we have discussed in Chapter 1, the information theoretic approach is an effective tool to quantitatively measure the amount of information flow induced by the change of morphology. After mounting suitable sensors to measure the state of the muscles (length or pressure), we hypothesize that information transfers and the associations between the variables (control signals and sensory information) brought about by the interaction with the environment can quantitatively reflect how the energy was transferred and stored during locomotion. This topic will be further studied in the future.

\subsubsection{Accuracy of the Spine Reservoir}

A compliant spine structure in the Kitty robot can serve as a computational resource to achieve different behaviors, such as bounding gait, trotting gait, and turning behavior, due to its intrinsic flexibility and nonlinearity (Chapter 6). However, we observed that for each gait pattern, the produced motor commands are noisy and cannot emulate the target commands precisely. This is mainly caused by the limitation of this platform when it starts to interact with the environment. For instance, the amount and quality of the teaching data available in physical platform are much lower than in comparable simulated work.

A possible solution to reduce the error between the target and the output signals is to optimize 
the physical spine structure by employing more asymmetrical features. This is inspired by the animals of which the spine morphology is asymmetrical in some cases, e.g., asymmetrical muscle stiffness, and the shape of the spinal column [Alexander and Jayes, 1981]. These properties could increase the diversity and nonlinearity of the spine reservoir and therefore improve its performance. In addition, to better reflect the spine dynamics in the sensory time series, the number and location of sensors embedded in the spine will be explored and optimized for future work.

\subsubsection{Gait Switching based on Morphological Computation}

We have already demonstrated that a compliant spine as a computational source can generate different locomotion behaviors by using one fixed physical body in the Kitty robot (Chapter 6). Another future research direction is to explore whether gait switching could be achieved in our framework [Owaki et al., 2012; Ijspeert et al., 2007]. There are two possibilities worth investigating. One possible scenario would be to explore ways to embed multiple gaits with a single fixed linear readout and a feedback loop by introducing an input signal corresponding to each gait to the spine. The signal acts as an initiation signal (or a control signal) for the gait switching and would be provided either as an external or internal control command [Hauser et al., 2012]. Especially in our contexts, the signals can be mechanical, such as an intentional movement of a head or a tail of the robot's body, or can be generated from an environmental change, e.g., the change from flat terrain to rough terrain. The other possibility is to integrate conventional reservoir computing (e.g., echo state network) to morphological computation. Such a systematic scheme would help to investigate how the body and the brain cooperate to solve a task. The simple starting point is to add the nodes of an echo state network one by one to the existing morphological computation to check at which level the gait switching could be achieved. This will help us to understand their roles in computation.

\subsection{Conclusion}

A spine is of crucial importance in the vertebrate locomotion. This thesis has explored the possibility of a spine to be the main driving force for locomotion in vertebrate animals. The contribution of this work is threefold: (i) development of a series of real robots and simulated agent driven by an actuated spine, (ii) validation of the spinal engine hypothesis, thus providing a different view of locomotion, (iii) elucidation of the effect of spine morphology in spine-driven locomotion.

A synthetic approach was followed by constructing a series of robots to study the role of the spine and its morphological effect on locomotion. First, it was demonstrated that it is possible for an actuated spine to drive locomotion in quadruped robots without leg actuation. Apart from it, we found that the locomotion is greatly facilitated by properly using spine morphology features 
inspired by the biology (e.g., the number of spinal joints, the stiffness distribution, the shape, and the actuation type of the spine, etc.). The obtained results are basically in agreement with biological findings, which reveals how such properties affect locomotion in nature. Furthermore, we observed that a soft compliant spine together with linear readouts and feedback can serve as a computational resource to generate different gait patterns in the context of morphological computation. 


\section{Bibliography}

[Alexander, 1982] Alexander, R. M. (1982). Locomotion of animals. Blackie.

[Alexander, 1988] Alexander, R. M. (1988). Why mammals gallop. Journal of Zoology, 28:237-245.

[Alexander, 2002] Alexander, R. M. (2002). Tendon elasticity and muscle function. Comparative Biochemistry and Physiology Part A: Molecular \& Integrative Physiology, 133(4):1001 - 1011.

[Alexander, 2003] Alexander, R. M. (2003). Principles of animal locomotion. Princeton University Press.

[Alexander et al., 1985] Alexander, R. M., Dimery, N. J., and Ker, R. F. (1985). Elastic structures in the back and their role in galloping in some mammals. Journal of Zoology, 207(4):467-482.

[Alexander and Jayes, 1981] Alexander, R. M. and Jayes, A. S. (1981). Estimates of the bending moments exerted by the lumbar and abdominal muscles of some mammals. Journal of Zoology, 194(3):291-304.

[Allen et al., 2003] Allen, T., Quinn, R., Bachmann, R. J., and Ritzmann, R. E. (2003). Abstracted biological principles applied with reduced actuation improve mobility of legged vehicles. In 2003 IEEE/RSJ International Conference on Intelligent Robots and Systems (IROS), volume 2, pages 1370-1375.

[anatomyEXPERT, 2013a] anatomyEXPERT (2013a). Epaxial muscles. http://www . anatomyexpert.com/structure_detail/5201/.

[anatomyEXPERT, 2013b] anatomyEXPERT (2013b). Hypaxial muscles. http://www . anatomyexpert.com/structure_detail/5250/.

[BostonDynamics, 2012] BostonDynamics (2012). Little dog. http: / / www . bostondynamics . com/robot_littledog.html.

[BostonDynamics, 2013a] BostonDynamics (2013a). Big dog. http: / / www . bostondynamics . com/robot_bigdog.html. 
[BostonDynamics, 2013b] BostonDynamics (2013b). Cheetah robot. http://www . bostondynamics.com/robot_cheetah.html.

[Boszczyk et al., 2001] Boszczyk, B. M., Boszczyk, A. A., and Putz, R. (2001). Comparative and functional anatomy of the mammalian lumbar spine. The Anatomical Record, 264(2):157-168.

[Boxerbaum et al., 2008] Boxerbaum, A., Oro, J., Peterson, G., and Quinn, R. (2008). The latest generation whegs robot features a passive-compliant body joint. In 2008 IEEE/RSJ International Conference on Intelligent Robots and Systems (IROS), pages 1636-1641.

[Buchli and Ijspeert, 2008] Buchli, J. and Ijspeert, A. J. (2008). Self-organized adaptive legged locomotion in a compliant quadruped robot. Autonomous Robots, 25(4):331-347.

[Burgess and Practitioner, 2013] Burgess, R. J. and Practitioner, F. (2013). Evolution of spine. http: //www.efeld.com/evolution/.

[Caro, 1986] Caro, T. (1986). The functions of stotting in thomson's gazelles: some tests of the predictions. Animal Behaviour, 34(3):663-684.

[Cavagna et al., 1977] Cavagna, G. A., Heglund, N. C., and Taylor, C. R. (1977). Mechanical work in terrestrial locomotion: two basic mechanisms for minimizing energy expenditure. American Journal of Physiology, 233(5):R243-61.

[Collins et al., 2005] Collins, S., Ruina, A., Tedrake, R., and Wisse, M. (2005). Efficient bipedal robots based on passive-dynamic walkers. Science, 307(5712):1082-1085.

[Culha and Saranli, 2011] Culha, U. and Saranli, U. (2011). Quadrupedal bounding with an actuated spinal joint. In 2011 IEEE International Conference on Robotics and Automation (ICRA), pages 1392-1397.

[Daan and Belterman, 1968] Daan, S. and Belterman, T. (1968). Lateral bending in locomotion of some lower tetrapods. In Proceedings of the Koninklijke Nederlandse Akademie van Wetenschappen, pages 245-266.

[Dickinson et al., 2000] Dickinson, M. H., Farley, C. T., Full, R. J., Koehl, M. A. R., Kram, R., and Lehman, S. (2000). How animals move: An integrative view. Science, 288(5463):100-106.

[English, 1980] English, A. W. (1980). The functions of the lumbar spine during stepping in the cat. Journal of Morphology, 165:55-66.

[Fife et al., 2001] Fife, M. M., Bailey, C. L., Lee, D. V., and Carrier, D. R. (2001). Function of the oblique hypaxial muscles in trotting dogs. The Journal of Experimental Biology, 204(13):23712381. 
[Folkertsma et al., 2012] Folkertsma, G., Kim, S., and Stramigioli, S. (2012). Parallel stiffness in a bounding quadruped with flexible spine. In 2012 IEEE/RSJ International Conference on Intelligent Robots and Systems (IROS), pages 2210-2215.

[Fukuoka et al., 2010] Fukuoka, Y., Katabuchi, H., and Kimura, H. (2010). Dynamic locomotion of quadrupeds Tekken 3\&4 using simple navigation. Journal of Robotics and Mechatronics, 22(1):3642.

[Gabrielli and von Karman, 1951] Gabrielli, G. and von Karman, T. (1951). What price speed? Journal of the American Society for Naval Engineers, 63:188-200.

[Gadow, 1933] Gadow, H. (1933). Evolution of the vertebral column. Cambridge University Press.

[Gal, 1992] Gal, J. M. (1992). Mammalian spinal biomechanics II. intervertebral lesion experiments and mechanisms of bending resistance. The Journal of Experimental Biology, 174:281-297.

[Gracovetsky, 1985] Gracovetsky, S. (1985). An hypothesis for the role of the spine in human locomotion: a challenge to current thinking. Journal of Biomedical Engineering, 7(3):205-216.

[Gracovetsky, 1989] Gracovetsky, S. (1989). The spinal engine. Springer.

[Gracovetsky and Iacono, 1987] Gracovetsky, S. and Iacono, S. (1987). Energy transfers in the spinal engine. Journal of Biomedical Engineering, 9(2):99-114.

[Hackert, 2002] Hackert, R. (2002). Dynamics of the pikas' half-bound - spinal flexion contributes to dynamic stability. aspects of sagittal bending of the spine in small mammals. PhD thesis, BiologicalPharmaceutical Faculty, Friedrich-Schiller-University of Jena, Germany.

[Hackert et al., 2006] Hackert, R., Schilling, N., and Fischer, M. S. (2006). Mechanical selfstabilization, a working hypothesis for the study of the evolution of body proportions in terrestrial mammals. Comptes Rendus Palevol, 5:541-549.

[Hackert et al., 2001] Hackert, R., Witte, H., and Fischer, M. (2001). Dynamics of half-bound and spinal flexion. In Motion Systems 2001: Collected short papers of the Innovationkolleg Bewegungssysteme Friedrich-Schiller Universitat Jena, Technische Universitat Ilmenau, pages 137-141.

[Han et al., 2013] Han, K.-S., Rohlmann, A., Zander, T., and Taylor, W. R. (2013). Lumbar spinal loads vary with body height and weight. Medical Engineering \& Physics, 35(7):969 - 977.

[Hansen et al., 2006] Hansen, L., de Zee, M., Rasmussen, J., Andersen, T. B., Wong, C., and Simonsen, E. B. (2006). Anatomy and biomechanics of the back muscles in the lumbar spine with reference to biomechanical modeling. Spine, 31(17):1888-1899.

[Hauser et al., 2011] Hauser, H., Ijspeert, A., Füchslin, R., Pfeifer, R., and Maass, W. (2011). Towards a theoretical foundation for morphological computation with compliant bodies. Biological Cybernetics, 105(5-6):355-370. 
[Hauser et al., 2012] Hauser, H., Ijspeert, A., Füchslin, R., Pfeifer, R., and Maass, W. (2012). The role of feedback in morphological computation with compliant bodies. Biological Cybernetics, 106(10):595-613.

[Hildebrand, 1959] Hildebrand, M. (1959). Motions of the running cheetah and horse. Journal of the Royal Society Interface, 40(4):481-495.

[Hosoda et al., 2008] Hosoda, K., Takuma, T., Nakamoto, A., and Hayashi, S. (2008). Biped robot design powered by antagonistic pneumatic actuators for multi-modal locomotion. Robotics and Autonomous Systems, 56(1):46-53.

[Howell, 1944] Howell, A. B. (1944). Speed in animals: Their specialization for running and leaping. University of Chicago Press.

[Iida et al., 2009] Iida, F., Minekawa, Y., Rummel, J., and Seyfarth, A. (2009). Toward a human-like biped robot with compliant legs. Robotics and Autonomous Systems, 57(2):139-144.

[Ijspeert, 2008] Ijspeert, A. J. (2008). Central pattern generators for locomotion control in animals and robots: A review. Neural Networks, 21(4):642 - 653.

[Ijspeert et al., 2007] Ijspeert, A. J., Crespi, A., Ryczko, D., and Cabelguen, J.-M. (2007). From swimming to walking with a salamander robot driven by a spinal cord model. Science, 315(5817):1416-1420.

[Institute, 2013] Institute, T. C. C. S. (2013). Understanding spinal anatomy: Regions of the spine-cervical, thoracic, lumbar, sacral. http://www.coloradospineinstitute.com/ subject . php?pn=anatomy-spinalregions14.

[Jaeger and Haas, 2004] Jaeger, H. and Haas, H. (2004). Harnessing nonlinearity: Predicting chaotic systems and saving energy in wireless communication. Science, 304(5667):78-80.

[Jusufi et al., 2008] Jusufi, A., Goldman, D. I., Revzen, S., and Full, R. J. (2008). Active tails enhance arboreal acrobatics in geckos. Proceedings of the National Academy of Sciences, 105(11):42154219.

[Kuriyama et al., 2009] Kuriyama, S., Ding, M., Kurita, Y., Ogasawara, T., and Ueda, J. (2009). Flexible sensor for mckibben pneumatic actuator. In 2009 IEEE Sensors, pages 520-525.

[Leeser, 1996] Leeser, K. F. (1996). Locomtotion experiments on a planar quadruped robot with articulated back spine. Master's thesis, Massachusetts Institute of Technology.

[Lewis and Bekey, 2002] Lewis, M. A. and Bekey, G. A. (2002). Gait adaptation in a quadruped robot. Autonomous Robots, 12(3):301-312. 
[Libby et al., 2012] Libby, T., Moore, T. Y., Chang-Siu, E., Li, D., Cohen, D. J., Jusufi, A., and Full, R. J. (2012). Tail-assisted pitch control in lizards, robots and dinosaurs. Nature, 481(7380):181184 .

[Long, 1997] Long, J. (1997). Locomotor design of dolphin vertebral columns: bending mechanics and morphology of Delphinus delphis. Journal of Experimental Biology, 200(1):65-81.

[Long et al., 1997] Long, J., Pabst, D. A., Shepherd, W. R., William, and Mclellan, A. (1997). Locomotor design of dolphin vertebral columns: bending mechanics and morphology of delphinus delphis. The Journal of Experimental Biologoy, 200:65-81.

[Lungarella et al., 2005] Lungarella, M., Pegors, T., Bulwinkle, D., and Sporns, O. (2005). Methods for quantifying the informational structure of sensory and motor data. Neuroinformatics, 3(3):243-262.

[Lungarella and Sporns, 2006] Lungarella, M. and Sporns, O. (2006). Mapping information flow in sensorimotor networks. PLoS Computational Biology, 2(10):1301-1312.

[Maass et al., 2002] Maass, W., Natschläger, T., and Markram, H. (2002). Real-time computing without stable states: A new framework for neural computation based on perturbations. Neural computation, 14(11):2531-2560.

[Macpherson and Ye, 1998] Macpherson, J. M. and Ye, Y. (1998). The cat vertebral column: stance configuration and range of motion. Experimental Brain Research, 119(3):324-332.

[Meyer and Guillot, 2008] Meyer, J.-A. and Guillot, A. (2008). Biologically-inspired robots. Springer.

[Minetti et al., 1999] Minetti, A., Ardigò, L., and Reinach, E.and Saibene, F. (1999). The relationship between mechanical work and energy expenditure of locomotion in horses. The Journal of Experimental Biology, 202:2329-2338.

[Mizuuchi et al., 1999] Mizuuchi, I., Matsuki, T., Inaba, M., and Inoue, H. (1999). GA-based motion generation for quadruped robot which has soft spine structure. In 17th Annual Conference of the Robotics Society of Japan, pages 199-200.

[Moon, 2001] Moon, B. (2001). Snake locomotion. http://www.ucs.louisiana.edu/ rorm2286/locomotn.htm.

[Moore and Roy, 2011] Moore, N. A. and Roy, W. A. (2011). Rapid Review Gross and Developmental Anatomy. Elsevier.

[Nakajima et al., 2011] Nakajima, K., Li, T., Sumioka, H., Cianchetti, M., and Pfeifer, R. (2011). Information theoretic analysis on a soft robotic arm inspired by the octopus. In 2011 IEEE International Conference on Robotics and Biomimetics (ROBIO), pages 110-117. 
[NASS, 2013] NASS (2013). Spinemotion. http://www.knowyourback.org/Pages/ Definitions/Anatomyspine/MotionSegment.aspx.

[Owaki et al., 2012] Owaki, D., Morikawa, L., and Ishiguro, A. (2012). Listen to body's message: Quadruped robot that fully exploits physical interaction between legs. In 2012 IEEE/RSJ International Conference on Intelligent Robots and Systems (IROS), pages 1950-1955.

[Pal and Sherk, 1988] Pal, G. and Sherk, H. (1988). The vertical stability of the cervical spine. Spine, 13(5):447-449.

[Palis et al., 2005] Palis, F., Rusin, V., Schmucker, U., Schneider, A., and Zavgorodniy, Y. (2005). Walking robot with articulated body and force controlled legs. In The 3rd International Symposium on Adaptive Motion of Animals and Machines (AMAM2005).

[Pfeifer and Bongard, 2007] Pfeifer, R. and Bongard, J. (2007). How the body shapes the way we think: a new view of intelligence. The MIT Press.

[Pfeifer et al., 2007] Pfeifer, R., Lungarella, M., and Iida, F. (2007). Self-organization, embodiment, and biologically inspired robotics. Science, 318(5853):1088-1093.

[Poulakakis et al., 2005] Poulakakis, I., Smith, J. A., and Buehler, M. (2005). Modeling and experiments of untethered quadrupedal running with a bounding gait: the Scout II robot. The International Journal of Robotics Research, 24(4):239-256.

[Pridmore, 1992] Pridmore, P. (1992). Trunk movements during locomotion in the marsupial monodelphis domestica (didelphidae). Journal of Morphology, 211(2):137-146.

[Provenzano et al., 2001] Provenzano, P., Lakes, R., Keenan, T., and Ray anderby, J. (2001). Nonlinear ligament viscoelasticity. Annals of Biomedical Engineering, 29:908-914.

[Raibert, 2000] Raibert, M. (2000). Legged robots that balance. The MIT Press.

[Roos, 1964] Roos, P. (1964). Lateral bending in newt locomtion. In Proceedings of the Koninklijke Nederlandse Akademie van Wetenschappen, pages 223-232.

[Sakai et al., 2007] Sakai, Y., Sato, T., Owaki, D., and Ishiguro, A. (2007). Realization of stable quadruped gait transition by changing body stiffness. In Proceedings of the 2007 JSME Conference on Robotics and Mechatronics, pages 2A1-2A10.

[Schilling, 2011] Schilling, N. (2011). Evolution of the axial system in craniates: morphology and function of the perivertebral musculature. Frontiers in Zoology, 8(4):1-19.

[Schilling and Carrier, 2010] Schilling, N. and Carrier, D. R. (2010). Function of the epaxial muscles in walking, trotting and galloping dogs: implications for the evolution of epaxial muscle function in tetrapods. The Journal of Experimental Biology, 213(9):1490-1502. 
[Schilling and Hackert, 2006] Schilling, N. and Hackert, R. (2006). Sagittal spine movements of small therian mammals during asymmetrical gaits. The Journal of Experimental Biology, 209:3925-3939.

[Schroer et al., 2004] Schroer, R., Boggess, M., Bachmann, R. J., Quinn, R., and Ritzmann, R. E. (2004). Comparing cockroach and whegs robot body motions. In 2004 IEEE International Conference on Robotics and Automation (ICRA), volume 4, pages 3288-3293.

[Sheikh, 2013] Sheikh, F. I. (2013). Towards fast running: Open-loop speed and direction control of a single-legged hopper. In 2013 IEEE/RSJ International Conference on Intelligent Robots and Systems (IROS), pages 5114-5120, Tokyo, Japan. IEEE.

[Shirazi-Adl, 1994] Shirazi-Adl, A. (1994). Biomechanics of the lumbar spine in sagittal/lateral moments. Spine, 19(21):2407-2414.

[Sumioka et al., 2010] Sumioka, H., Yoshikawa, Y., and Asada, M. (2010). Reproducing interaction contingency toward open-ended development of social actions: Case study on joint attention. IEEE Transactions on Autonomous Mental Development, 2(1):40-50.

[Takuma and Masuda, 2010] Takuma, T.and Ikeda, M. and Masuda, T. (2010). Facilitating multimodal locomotion in a quadruped robot utilizing passive oscillation of the spine structure. In 2010 IEEE/RSJ International Conference on Intelligent Robots and Systems (IROS), pages 4940-4945.

[Taylor, 1978] Taylor, C. R. (1978). Why change gaits? Recruitment of muscles and muscle fibres as a function of speed and gait. American Zoologist, 18:153-161.

[Taylor, 2012] Taylor, T. (2012). Lumbar spine. http://www. innerbody . com/anatomy/ skeletal/lumbar-vertebrae-lateral.

[Townsend et al., 1983] Townsend, H. G., Leach, D. H., and Fretz, P. B. (1983). Kinematics of the equine thoracolumbar spine. Equine veterinary journal, 15(2):117-122.

[Trimmera, 2008] Trimmera, B. A. (2008). New challenges in biorobotics: Incorporating soft tissue into control systems. Applied Bionics and Biomechanics, 5(3):119-126.

[Tsujita et al., 2008] Tsujita, K., Kobayashi, T., Inoura, T., and Masuda, T. (2008). Gait transition by tuning muscle tones using pneumatic actuators in quadruped locomotion. In 2008 IEEE/RSJ International Conference on Intelligent Robots and Systems (IROS), pages 2453-2458.

[Tsujita and Miki, 2011] Tsujita, K. and Miki, K. (2011). A study on trunk stiffness and gait stability in quadrupedal locomotion using musculoskeletal robot. In 2011 15th International Conference on Advanced Robotics (ICAR), pages 316-321.

[Wakimoto et al., 2005] Wakimoto, S., Suzumori, K., and Kanda, T. (2005). Development of intelligent mckibben actuator with built-in soft conductive rubber sensor. In The 13th International Conference on Solid-State Sensors, Actuators and Microsystems, 2005, volume 1, pages 745-748. 
[Webb, 2002] Webb, B. (2002). Robots in invertebrate neuroscience. Nature, 417(6886):359-363.

[White and Panjabi, 1990] White, A. A. and Panjabi, M. M. (1990). Clinical Biomechanics of the Spine. Lippincott Williams \& Wilkins.

[Wilson et al., 2013] Wilson, A. M., Lowe, J. C., Roskilly, K., Hudson, P. E., Golabek, K. A., and McNutt, J. W. (2013). Locomotion dynamics of hunting in wild cheetahs. Nature, 498(7453):185189.

[Zhao et al., 2013a] Zhao, Q., Ellenberger, B., Sumioka, H., Sandy, T., and Pfeifer, R. (2013a). The effect of spine actuation and stiffness on a pneumatically-driven quadruped robot for cheetahlike locomotion (to appear). In 2013 IEEE International Conference on Robotics and Biomimetics (ROBIO).

[Zhao et al., 2013b] Zhao, Q., Nakajima, K., Sumioka, H., Hauser, H., and Pfeifer, R. (2013b). Spine dynamics as a computational resource in spine-driven quadruped locomotion. In 2013 IEEE/RSJ International Conference on Intelligent Robots and Systems (IROS), pages 1445-1451.

[Zhao et al., 2012] Zhao, Q., Nakajima, K., Sumioka, H., Yu, X., and Pfeifer, R. (2012). Embodiment enables the spinal engine in quadruped robot locomotion. In 2012 IEEE/RSJ International Conference on Intelligent Robots and Systems (IROS), pages 2449-2456.

[Zhao et al., 2014] Zhao, Q., Sumioka, H., Nakajima, K., Yu, X., and Pfeifer, R. (2014). Spine as an engine: Effect of spine morphology on spine-driven quadruped locomotion. Advanced Robotics, 28(0):367-378.

[Zhao et al., 2011] Zhao, Q., Sumioka, H., and Pfeifer, R. (2011). The effect of morphology on the spinal engine driven locomotion in a quadruped robot. In The 5th International Symposium on Adaptive Motion of Animals and Machines (AMAM2011), pages 51-52. 


\section{Appendix A}

\section{The Effect of Morphology on the Spinal Engine Driven Locomotion in a Quadruped Robot}

Zhao, Q., Sumioka, H., and Pfeifer, R.(2011). The Effect of Morphology on the Spinal Engine Driven Locomotion in a Quadruped Robot, In the 2011 IEEE International Symposium on Adaptive Motion of Animals and Machines (AMAM), pp. 51-52.

This is the final accepted version. Final version of the article can be found at http:/ /adaptivemotion.org/AMAM2011/papers/s234.pdf. 


\title{
The Effect of Morphology on the Spinal Engine Driven Locomotion in a Quadruped Robot
}

\author{
Qian Zhao, Hidenobu Sumioka, and Rolf Pfeifer \\ Artificial Intelligence Laboratory \\ Department of Informatics, University of Zurich \\ Andreasstrasse 15, CH-8050 Zurich, Switzerland \\ E-mail: (zhao, sumioka, pfeifer)@ifi.uzh.ch
}

\begin{abstract}
The biological hypothesis of spinal engine states that the locomotion is mainly achieved by the spine, while legs only serve as assistance. Inspired by this spinal engine hypothesis, a compliant, multi-DOF, biologically inspired spine has been developed and embedded into a quadruped robot without actuation on legs. The experimental results support this spinal engine hypothesis and reveal that this kind of robot can achieve rapid, stable, and even dynamical locomotion by appropriately tuning the spine’s morphological parameters, e.g., rearranging the silicone blocks.
\end{abstract}

Keywords: Spinal engine, Locomotion, Quadruped robot

\section{INTRODUCTION}

Over the past decades, it has been widely accepted that locomotion is generally achieved by the coordination of the legs and the spine is only considered to be carried along in a more or less passive way [1] This popular hypothesis has been accepted by most of robotics researchers as well as biologists. A considerable amount of research has been conducted on legged robots with little consideration on their spines [2]. However, Gracovetsky has proposed an alternative hypothesis with an emphasis on the spinal engine in human locomotion, i.e., locomotion is firstly achieved by the motion of the spine; the limbs came after, as an improvement but not a substitute [3]. Then, he extended this hypothesis to quadruped animals featuring flexion-extension spinal movement [4]. This implies that the spine is the key structure in locomotion. Recently, some robotics researchers came to realize the importance of the spine, but most of them still consider the spine as an assistant element to enhance the capability of locomotion [5], [6].

In this paper, we proposed a biologically inspired spine model and its application to a real quadruped robot to investigate its role in locomotion. Preliminary experimental results support the hypothesis of spinal engine and reveal that rapid and dynamical locomotion can be achieved only by actuating the biologically inspired tendon-driven actuated spine, without taking the actuation of the legs into account. More morphologies of the spine are explored and the results suggest that the locomotion behavior can be changed by tuning the morphology of the spine.

\section{BIOLOGICALLY INSPIRED SPINE MODEL}

Fig.1 (a) shows an artificial spine endowed with biological characteristics. It consists of cross-shaped rigid vertebrae made of ABS plastic, silicon blocks and cables driven by motors. The vertebrae are separated by the silicon blocks, which work as intervertebral discs.
They are connected by a cable through themselves and the silicon blocks. The four driven cables are pulled respectively by four RC motors, which can control the stiffness and movement of the spine. In this design, multiple socket-ball joints (Fig.1 (b)) are taken to produce a more versatile posture and a wider motion space. The resulted spine can be bent in all directions within a certain predefined angle and form asymmetrical complex configuration by rearranging the silicone blocks in between.

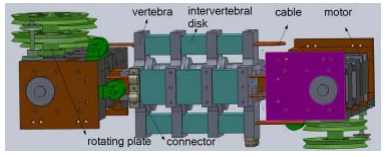

(a)

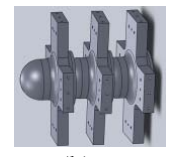

(b)
Fig. 1 The whole spine structure (a) and its socket-ball joints (b).

\section{DESIGN OF THE QUADRUPED ROBOT}

The developed artificial spine is embedded into a quadruped robot (29 cm wide, 23 or $25 \mathrm{~cm}$ long, $20 \mathrm{~cm}$ high and $1.1 \mathrm{~kg}$ ). There are 3 linear springs in each stick-shaped leg to cushion shock from the ground. The legs are fixed to the body. The bottom of foot is glued with asymmetrical friction material to control the walking direction. Sine waves with tunable parameters are taken as control signals for 4 motors to generate the spinal movement.

\section{EXPERIMENTAL RESULTS}

To better understand the role of spine in locomotion and the correlation between its morphological property and locomotion behavior, a series of experiments were conducted under the condition of different spinal structures which differ in the shape and the stiffness distribution. During the experiments, several control parameter sets were tested for 5 trials and the best one was chosen. Fig.2 shows two robots equipped with symmetrical rectangle and rhombus shaped spines. 


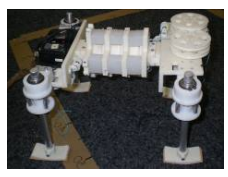

(a)

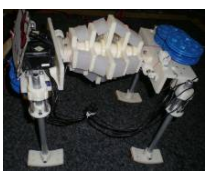

(b)
Fig.2 Robots equipped with rectangle (a) and rhombus (b) shaped tendon-driven actuated spine.

\subsection{Moving forward}

Table. 1 shows that the robot is able to walk forward rapidly with stable and reproducible performance. We have also observed the shape of the spine does not affect much on its speed. Fig.3 exhibits the symmetrical, periodical flexion-extension spinal movements generating power to locomotion. However, its feet slide on the ground due to the lack of ground clearance.

Table. 1 Results of the moving forward performance

\begin{tabular}{|c|c|c|}
\hline Symmetrical spine shape & Rectangle & Rhombus \\
\hline Ave speed (cm/s) & 14.5 & 11.7 \\
\hline Std speed & 0.3 & 0.3 \\
\hline
\end{tabular}
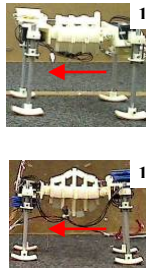
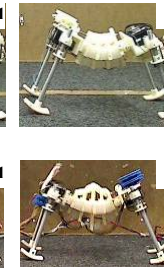

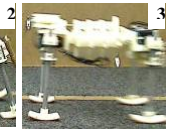
(a)
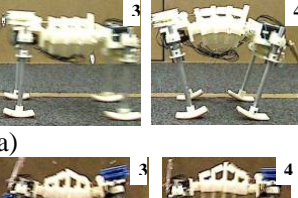

(b)
Fig.3 Sequential pictures of the robot's locomotion with the rectangle (a) and rhombus (b) shaped spine under the same control set. The red arrows represent the walking direction.

\section{2 Turning left/right}

Table. 2 shows the robot is able to turn right or left stably with the symmetrical rectangle shaped spine by introducing the lateral movement in addition to the flexion-extension movements. The speeds slightly differ due to the manufacture and assembly error. Table. 2 Results of turning performance

\begin{tabular}{|c|c|c|}
\hline Rectangle-shaped spine & Turing Right & Turing Left \\
\hline Ave angular speed $(\% / \mathrm{s})$ & 6.2 & 5.1 \\
\hline Std angular speed & 1.0 & 0.5 \\
\hline
\end{tabular}

\section{3 Dynamical movement}

In this experiments, the asymmetrical spine morphologies are introduced where the silicones in the fore part of the spine were partially taken out in the above two morphologies. Fig.4 exhibits more dynamical lifting up movement based on these two new morphologies due to the asymmetrical arrangement of the silicone blocks. However, the rhombus shaped spine can lift up not only the fore legs, but the rear legs, which might be explained by the more asymmetrical stiffness distribution formed by this shape.
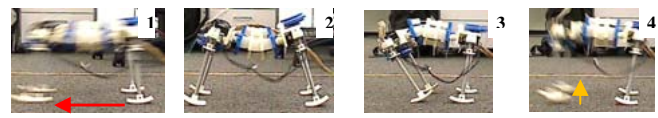

(a)
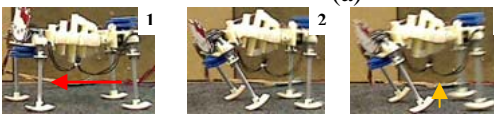

Fig.4 Sequential pictures of the robot's locomotion with the asymmetrical rectangle (a) and rhombus (b) shaped spine under the same control set. The yellow arrows represent the movement of lifting up.

\section{CONCLUSIONS}

A novel highly-compliant, multi-joint artificial spine inspired by biology has been developed and applied to a quadruped robot to test the biological hypothesis of spinal engine. Preliminary experimental results showed that the rapid and stable forward moving can be achieved when the silicon blocks are distributed symmetrically in the spine, whereas more dynamical movement can be observed by taking asymmetrical distribution of silicon blocks. This phenomenon has been observed in the cases of both the rectangle and rhombus morphologies, but the robot equipped with rhombus-shaped spine can lift up rear legs, which might be interpreted by its spine's ability to generate more complex asymmetrical configuration. The sensitive turning performance in the rectangle case has been observed to further support the spinal engine hypothesis.

All the results emphasized the concept of spinal engine and demonstrated the possibility for a robot to achieve different locomotion modes by appropriately tuning the morphological parameters of the spine without taking the actuation of the legs into account.

\section{REFERENCES}

[1] R.M. Alexander, "Principles of Animal Locomotion", Princeton University Press, Princeton NJ, 2003.

[2] M. H. Raibert, "Legged robots", Communications of the ACM, 29(6):pp.499-514, 1986.

[3] S. A. Gracovetsky, "An hypothesis for the role of the spine in human locomotion: a challenge to current thinking”, J Biomed Eng, 7(3): pp. 205-216, 1985.

[4] S. A. Gracovetsky, "Energy transfers in the spinal engine”, J Biomed Eng, 9(2): pp. 99-114, 1987.

[5] T. Takuma, "Facilitating Multi-modal Locomotion in a Quadruped Robot utilizing Passive Oscillation of the Spine Structure", 2010 IEEE/RSJ International Conference on Intelligent Robots and Systems, ThBT5, 2010.

[6] K. Tsujita, "Gait Transition by Tuning Muscle Tones using Pneumatic Actuators in Quadruped Locomotion”, Proc. of IEEE/RSJ IROS2008, Vol. 1-3, pp. 2453-2458, 2008. 



\section{Appendix B}

\section{Embodiment Enables the Spinal Engine in Quadruped Robot Locomotion}

(C)2012 IEEE. Reprinted, with permission, from:

Zhao, Q., Nakajima, K., Sumioka, H., Yu, X., and Pfeifer, R.(2012). Embodiment Enables the Spinal Engine in Quadruped Robot Locomotion, In the 2012 IEEE/RSJ International Conference on Intelligent Robots and Systems (IROS), pp. 2449-2456.

This is the final accepted version. Final version of the article can be found at http:/ / ieeexplore.iee.org (doi=10.1109/IROS.2012.6386048). 


\title{
Embodiment Enables the Spinal Engine in Quadruped Robot Locomotion
}

\author{
Qian Zhao, Kohei Nakajima, Hidenobu Sumioka, Xiaoxiang Yu, and Rolf Pfeifer
}

\begin{abstract}
The biological hypothesis of spinal engine states that locomotion is mainly achieved by the spine, while the legs may serve as assistance. Inspired by this hypothesis, a compliant, multiple degree-of-freedom, biologically-inspired spine has been embedded into a quadruped robot, named Kitty, which has no actuation on the legs. In this paper, we demonstrate how versatile behaviors (bounding, trotting, and turning) can be generated exclusively by the spine's movements through dynamical interaction between the controller, the body, and the environment, known as embodiment. Moreover, we introduce information theoretic approach to quantitatively study the spine internal dynamics and its effect on the bounding gait based on three spinal morphologies. These three morphologies differ in the position of virtual spinal joint where the spine is easier to get bent. The experimental results reveal that locomotion can be enhanced by using the spine featuring a rear virtual spinal joint, which offers more freedom for the rear legs to move forward. In addition, the information theoretic analysis shows that, according to the morphological differences of the spine, the information structure changes. The relationship between the observed behavior of the robot and the corresponding information structure is discussed in detail.
\end{abstract}

\section{INTRODUCTION}

Legged robotics has attracted much more attention from robotic researchers due to its application in rough terrains in nature and in our living environment [1]. Over the past decades, it has been widely accepted that locomotion is generally achieved by the coordination of the legs, and the spine is only considered to be involved in a passive way. Most existing quadruped robots are very similar in their morphology and feature a single rigid body with four legs with individually actuated hips and knees [2]-[5].

However, the resulting locomotion behavior is much more constrained than its natural counterpart in terms of speed, energy efficiency, maneuverability, and adaptability to rough terrain. From a biological point of view, one of the major differences between robots and animals is the spine. It's function is not only to bear weight, but also to facilitate locomotion. For example, cheetah, the fastest animal on land, exhibits pronounced flexion-extension spinal movement and is able to reach a speed of $110 \mathrm{~km} / \mathrm{h}$ for a short dash.

Therefore, an alternative biological hypothesis has been proposed by S. A. Gracovetsky that emphasizes on the spinal

This research was funded by the European Community's Seventh Framework Programme FP7-ICT-248311(AMARSi).

Q. Zhao, K. Nakajima, H. Sumioka, X.Yu and R. Pfeifer are with Department of Informatics, University of Zurich, 8050, Zurich, Switzerland, email: $\{$ zhao/nakajima\}@ifi.uzh.ch

H. Sumioka has moved to Advanced telecommunications research institute international.

$\mathrm{X}$. Yu is also with Bio-Inspired Robotics Lab, Swiss Federal Institute of Technology Zurich, 8092 Zurich, Switzerland. engine, i.e., locomotion is first achieved by the motion of the spine; the limbs came after, as an improvement but not a substitute [6]. This hypothesis was then extended to quadruped animals featuring flexion-extension and lateral spinal movement [7], [8]. In addition, B. M. Boszczyk demonstrated that mammalian lumbar spines suggest an exact accommodation to specific biomechanical demands, i.e., spinal morphology depends on the locomotion mode in nature [9]. These biological findings indicate that the spine is a crucial element in locomotion.

Inspired by these findings, some robotics researchers came to realize the importance of the spine, but most only focused on the controller of the spinal joint, or regarded the spine as an assistant element to enhance locomotion [10]-[12]. All the aforementioned studies simply introduced a spinal joint connecting the front and hind part without further study on its morphological parameters. A recent study has demonstrated how spinal morphology affects a passive quadruped robot to go down a slope without external energy and has shed new light on the study of the spine in locomotion [13]. However, more versatile behaviors generated by the spine are expected to emerge and the effect of spinal morphology in locomotion needs to be studied and quantified. We know that this passive bounding behavior is generated through the embodied coupling between the body and the environment, but how the change of spinal morphologies modulates this embodied coupling is still unknown. Such an investigation is challenging because the coupling between controller, body, and environment [14], [15] is usually nonlinear and complex. One promising approach is to take information theoretic measures to quantify information structure in sensorimotor interactions because these measures can detect nonlinear relations between variables [16]-[21].

In this paper, we introduce a biologically-inspired multiple degree-of-freedom spine model [22], [23] to a real quadruped robot to demonstrate how versatile behaviors (bounding, trotting, and turning) can be generated by the spinal engine through embodied coupling between the controller, the body and the environment. Moreover, three different spinal morphological configurations are introduced to the robot. Then their effects in bounding and trotting gaits are analyzed. We further investigate the effect of these three spinal morphologies on the bounding gait based on information theoretic measures that are able to characterize the sensorimotor interactions. The results suggest that through the embodied interaction with the environment, in particular through sensorimotor coordination, information structure is induced in the sensory data. The correspondence between the 


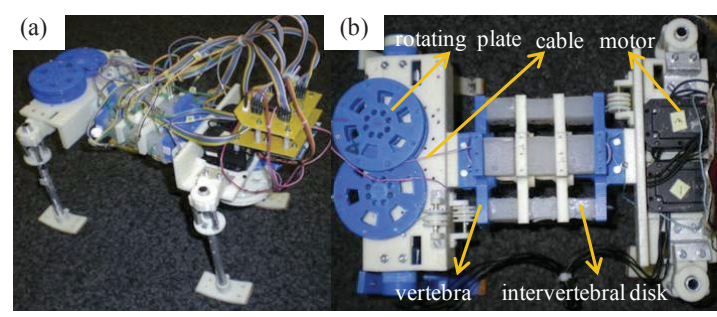

(c) rotating plate cable vertebra intervertebral disk motor

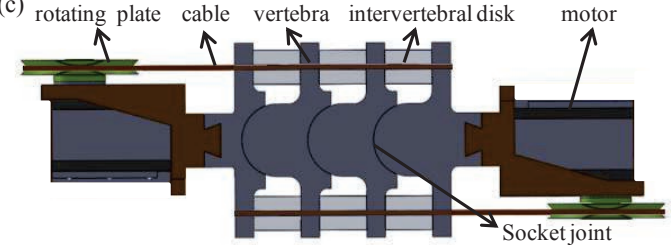

(d)

(e)

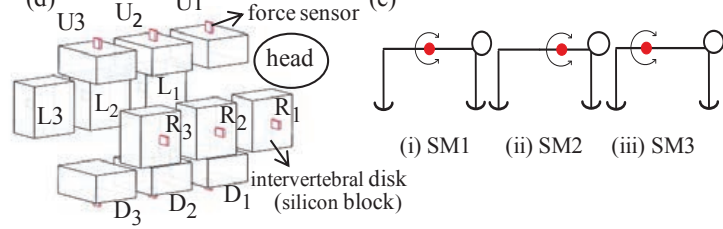

Fig. 1. (a) Kitty equipped with a tendon-driven spine. (b) A biologically inspired spine. (c) Cross section of the artificial spine: sagittal view. (d) The arrangement of sensors in the spine. Red cubic stands for the force sensor embedded in the silicon block. (e) Schematic representation of robot equipped with an actuated spine whose virtual joint is in the middle (i), front (ii) and the rear (iii) part of the body. The red point stands for the virtual spinal joint.

observed behaviors of the robot and the information structure is discussed in detail.

\section{EXPERIMENTS SETTING}

\section{A. Quadruped robot}

We built a robot, called Kitty, with a flexible actuated spine, to test the concept of the spinal engine based on the interaction between the controller, the body and the environment. An overview of the whole robot, spine structure, spine morphology, controller design, and data acquisition are presented in this section.

1) Overview of the whole robot: Kitty is equipped with a flexible spine (29 $\mathrm{cm}$ wide, $32 \mathrm{~cm}$ long, $20 \mathrm{~cm}$ high, and $1.4 \mathrm{~kg}$ ) (Fig. 1 (a)). Three linear springs are mounted in each stick-shaped leg to cushion the shock from the ground. The legs are fixed to the body and have no relative rotation with respect to it. The bottoms of the feet are glued with asymmetrical friction material to guide the walking direction.

2) Biologically-inspired spine structure: As an essential structure for both weight bearing and locomotion, the spine is subject to the conflict of providing maximal stability while maintaining crucial mobility. It consists of discrete bony elements, namely vertebrae, joined by passive ligaments and separated by intervertebral discs [24]. The Zygapophyseal joints are dynamically controlled by muscular activation.
Fig. 1 (b) shows an artificial spine endowed with biological characteristics. It consists of cross-shaped rigid vertebrae, made of acrylonitrile-butadiene-styrene (ABS) plastic, silicon blocks, and cables driven by motors. As shown in Fig. 1 (c), the vertebrae are separated by the silicon blocks, which work as intervertebral discs, and connected by four cables through themselves and the silicon blocks. The four cables are pulled respectively by four electronic motors through the rotating plates. In this design, multiple socket-ball joints are employed to produce a more versatile posture and a wider motion space (Fig. 1 (c)). The resulting spine can be bent in all directions within a certain predefined angle and form an asymmetrical complex configuration by rearranging the silicone blocks in between.

3) Spine morphologies: As we all know, some biological features, e.g., unevenly distributed muscles in the spine, asymmetrical muscle stiffness, and the shape of the spinal column [25], all have the possibility to make the body bend more in one direction and less in another direction. In order to study the effect of asymmetrical structure in locomotion, we design a virtual joint on spine and change its position with the aim of gaining a better understanding of the underlying mechanism of the spine.

We define the virtual spinal joint (VSJ) as a point where the spine is more likely to achieve wider bending movement. The position of the VSJ can be changed by removing some of the silicon blocks from the spine. Twelve silicon blocks are distributed in the spine and three at each side, as shown in Fig. 1(d). We name them by the combination of the direction, e.g., up (U), down (D), left (L), right (R) and the order, e.g., 1 (in the front), 2 (in the middle), 3 (in the rear).

We specify the position of the VSJ in the middle, if the silicon blocks fill in all the gaps between the vertebrae and distribute evenly along the spine (Fig. 1(e) (i)). The resultant morphology is named spinal morphology 1 (SM1). The morphology, without D1, L1, and R1, which tends to bend more in the front, is called spinal morphology 2 (SM2) (Fig. 1(e) (ii)). Spinal morphology 3 (SM3) is defined as the one without D3, L3, and R3 that bends easily in the rear part of the spine (Fig. 1(e) (iii)) .

Since only two silicon blocks are located in the downside of the spine in SM1 and SM2, their stiffness in this side is less than the one in SM1 which consists of three silicon blocks in the same side. Each side of spine is a complex system, because it is made up of three or two soft silicon blocks working as compression springs, and four rigid plastic segments. Therefore, we simplified the procedure to measure the stiffness in each side of spine as follows. When one side of the spine is pulled, the other three are relaxed. The cable connecting through this side is pulled by a motor to a target position $\left(\frac{2 \pi}{3}\right.$ radian) and the silicon blocks get compressed. We measured the force exerting on this side and its deformation. Next we calculated its stiffness by dividing the measured force by the deformation. We found that stiffness of the side with three silicon blocks is 0.5 $(\mathrm{N} / \mathrm{mm})$, while the one with two blocks is $0.33(\mathrm{~N} / \mathrm{mm})$. 
4) Controller design: Four electronic motors (Dynamixel RX 28) are mounted at the front and rear parts of the body to generate force to control the movement of the spine. The motors are serially connected to a PC by a USB2Dynamixel adapter. Position control is taken to drive the spine. The goal position should be set within a valid range from $-\frac{5 \pi}{6}$ to $+\frac{5 \pi}{6}$, due to the limitation of the motors. Sinusoid functions with tunable parameters are used as control signals for four motors to generate versatile spinal movement (Table I). We represent these motor commands as MU, ML, MR, and MD, according to the spinal parts they actuate, such as up, left, right, and down, respectively.

Each force sensor (FSR400) is inserted into a silicon block to collect force information generated by the compression of the spine (Fig. 1 (d)). The naming of the force sensors is the same as the silicon blocks. An Arduino Uno board [26] is used to collect analog data and transfer them to the computer.

\section{B. Information theoretic measures}

As we mentioned in Section I, the behavior of the robot is generated from reciprocal and dynamical coupling between the control, the body, and the environment. This means that any changes in the behavior of the robot can be characterized by changes of this coupling regime. In order to analyze this coupling, simulation models are often constructed to emulate the properties of the physical platform. However, in case of a physical platform that contains soft material, it is difficult to fine-tune a parameter, such as a collision coefficient and elasticity, even if the setting is simple. In such cases, the information theoretic approach can be effectively used to characterize the system in detail. It is intrinsically a modelfree approach and can be used for nonlinear time series. Thus, it has been recently used to characterize the coupling regime of robots [16]-[21].

Usually, an information theoretic approach makes extensive demands on the amount of data and preconditioning of them, such as fine-tuning parameters and data discretization, when applied to a continuous time series. In addition, it is often sensitive to noise, which limits the applications in a real-world physical experiment. In order to overcome this limitation, permutation entropy (PE) was proposed [27]. Permutation entropy quantifies the uncertainty of orderings between values, unlike the usual entropy which quantifies that of values themselves. Despite the differences between the procedures, it was proved that the $P E$ rate is equal to the

TABLE I

PARAMETERS FOR DIFFERENT BEHAVIORS

\begin{tabular}{|c|c|c|c|c|c|c|c|}
\hline Behavior & $A_{U}^{1} / A_{D}$ & $A_{R} / A_{L}$ & $F_{U D}^{2}$ & $F_{R L}$ & $\phi_{D}^{3}$ & $\phi_{R}$ & $\phi_{L}$ \\
\hline Bounding & $\frac{2 \pi}{3}$ & 0 & 0.01 & 0 & $\pi$ & 0 & 0 \\
\hline Trotting & 0 & $\frac{2 \pi}{3}$ & 0 & 0.01 & 0 & $\pi$ & 0 \\
\hline Turning right & $\frac{2 \pi}{3}$ & $\frac{2 \pi}{3}$ & 0.01 & 0.01 & $\pi$ & $\pi$ & 0 \\
\hline Turning left & $\frac{2 \pi}{3}$ & $\frac{2 \pi}{3}$ & 0.01 & 0.01 & $\pi$ & 0 & $\pi$ \\
\hline
\end{tabular}

${ }^{1} A_{U} / A_{D} / A_{R} / A_{L}$ : amplitude of each side of spine (radian)

${ }^{2} F_{U D} / F_{R L}$ : frequency of up-down/right-left (hertz).

${ }^{3} \phi_{R} / \phi_{L}$ : phase lag at each side relative to the upside signal (radian). usual entropy rate for any finite-state stationary stochastic process [28], [29]. The method is especially useful since it contains a natural symbolization procedure of values, which usually requires preprocessing to determine the appropriate bin size for the discretization of the acquired data. It was also shown that $P E$ is robust to noise, which is common to real-world time series [27].

Let $x_{t}$ represent a set of amplitude values of time series $x_{t}^{\prime}$ with a given embedding dimension $m$, and $\hat{x}_{t}$ as corresponding symbols. Based on the permutations of the values, $\hat{x}_{t}$ is generated as follows: $x_{t}=\left\{x_{t}^{\prime}, x_{t+1}^{\prime}, \ldots, x_{t+(m-1)}^{\prime}\right\}$, which are arranged in ascending order, $\left\{x_{t+\left(k_{t 1}-1\right)}^{\prime} \leq x_{t+\left(k_{t 2}-1\right)}^{\prime} \leq\right.$ $\left.\ldots \leq x_{t+\left(k_{t m}-1\right)}^{\prime}\right\}$. A symbol is thus defined as $\hat{x}_{t} \equiv$ $\left(k_{t 1}, k_{t 2}, \ldots, k_{t m}\right) \in X_{t}$. (Actually, for computational efficiency, we used the procedure introduced in [28], [29], which uses rank sequences. In [29], the use of the rank sequences is proved to be equal to the one presented here. It is useful to uniquely map $x_{t}$ onto $m$ ! possible permutations, including the case of equal amplitude values.) Based on the generated symbol $\hat{x}_{t}, P E\left(H\left(X_{t}\right)\right)$ is defined as

$$
H\left(X_{t}\right)=-\sum_{\hat{x}_{t}} p\left(\hat{x}_{t}\right) \log p\left(\hat{x}_{t}\right)
$$

where $p\left(\hat{x}_{t}\right)$ is a single probability associated with $\hat{x}_{t}$. In this paper, based on $P E$, we adopt two information theoretic measures and in each case, $m=6$ throughout this paper.

1) Permutation mutual information: Mutual information is a general measure of association between two or more random variables, naturally encompassing both linear and nonlinear dependencies [30]. We also consider the permutation version of mutual information in this paper. Permutation mutual information $(P M I)$ measures statistical independence as:

$$
P M I\left(X_{t}, Y_{t}\right)=\sum_{\hat{x_{t}}, \hat{y_{t}}} p\left(\hat{x}_{t}, \hat{y}_{t}\right) \log \frac{p\left(\hat{x_{t}}, \hat{y_{t}}\right)}{p\left(\hat{x_{t}}\right) p\left(\hat{y}_{t}\right)},
$$

where $p\left(\hat{x}_{t}, \hat{y}_{t}\right)$ is a joint probability associated with states $\hat{x}_{t}$ and $\hat{y}_{t}$. For statistically independent distributions, $p\left(\hat{x}_{t}, \hat{y}_{t}\right)=$ $p\left(\hat{x}_{t}\right) p\left(\hat{y}_{t}\right)$ and $\operatorname{PMI}\left(X_{t}, Y_{t}\right)=0$. If there exist statistical dependencies, $\operatorname{PMI}\left(X_{t}, Y_{t}\right)>0$.

2) Symbolic transfer entropy: Transfer entropy (TE) is a measure of the information transfer from the driving system $(Y)$ to the responding system $(X)$ [31]. The permutation version of transfer entropy is proposed in [32] and is called Symbolic Transfer Entropy (STE), expressed as:

$$
\operatorname{STE}(Y \rightarrow X)=\sum_{\hat{x}_{t+1}, \hat{x}_{t}, \hat{y}_{t}} p\left(\hat{x}_{t+1}, \hat{x}_{t}, \hat{y}_{t}\right) \log \frac{p\left(\hat{x}_{t+1} \mid \hat{x}_{t}, \hat{y}_{t}\right)}{p\left(\hat{x}_{t+1} \mid \hat{x}_{t}\right)},
$$

where the index $\operatorname{STE}(Y \rightarrow X)$ indicates the influence of $\hat{y}_{t}$ on $\hat{x}_{t+1}$ and can thus be used to detect the directed information transfer from $Y$ to $X$. In other words, STE somehow measures how well we can predict the state transition of the system $X$ by knowing a state of system $Y$. STE is nonnegative; any information transfer between the two variables results in $S T E>0$. If the state of $\hat{y}_{t}$ has no influence on the transition probabilities from $\hat{x}_{t}$ to $\hat{x}_{t+1}$, or if the two time series are completely synchronized, $S T E=0$. Recently, 
TE and STE have been proved to be equivalent if they are considered as the rates within the realm of finite-alphabet stationary ergodic Markov processes [33] and hidden Markov models with ergodic internal processes [34].

\section{EXPERIMENTS}

Two sets of experiments were conducted. The first one was with SM1. The goal is to show the versatility of spinegenerated behaviors (bounding, trotting, and turning). The second set studied the effect of three spinal morphologies (SM1, SM2, and SM3) on the bounding and trotting gaits.

During all the experiments, the average speed and significant difference were calculated based on three trials for each experiment, and one trial consists of 1200 time steps. The unit representing "time" in this paper is one sensing and actuation loop of the control program. One cycle of the motor command is 100 time steps and 12 cycles were run in each trial.

\section{A. Versatile behaviors}

1) Bounding/Flexion-extension spinal movement: The bounding gait is generated by spinal flexion-extension movements, which are achieved by pulling cables located at the upside and downside of the spine in an alternate way. The side cables are kept the natural length without stretching and relaxing. Fig. 2(a) shows the dynamics of the middle spine during one cycle of the bounding gait. The value in the vertical axis in Fig. 2 is a normalized sensory output voltage, which represents the force applied to the sensor. The applied force $\mathrm{F}$ (Newton) can be computed as $F=\frac{10 y}{256-y}$, where y is the value of the vertical axis. We use y to express the sensory response throughout this paper. We can see that the up and down sensors (red and purple lines in Fig. 2 (a)) are compressed alternately as a result of motor commands.

Fig. 3(a) shows symmetrical, periodic flexion-extension spinal movements generating power for locomotion. The robot is able to move forward at a speed of $5.76 \mathrm{~cm} / \mathrm{s}$ with a standard deviation of 0.09 (Fig. 4(a)). However, as can be seen from Fig. 3(a), its feet slide on the ground due to the lack of ground clearance.

2) Trotting/Lateral bending: The trotting gait is generated by the spinal lateral bending movement, which is produced by pulling side cables alternately while keeping the up and down cables the natural length. Fig. 2(b) shows the middle spine internal dynamics induced by the motor pattern. The spine exhibits symmetrical and periodic lateral bending movement and generates the trotting gait (Fig. 3(b)). The robot is able to trot at a speed of $1.7 \mathrm{~cm} / \mathrm{s}$ with a standard deviation of 0.1 (Fig. 4(b)). We observed that the speed can reach $3.0 \mathrm{~cm} / \mathrm{s}$ by increasing the frequency to $0.025 \mathrm{~Hz}$.

3) Turning: The turning behavior emerges if the lateral spinal movement is introduced to the spine while flexionextension spinal movement is retained. The only difference between turning right and left is the flip between $\phi_{R}$ and $\phi_{L}$, which are the phase lags with respect to the upside of the spine. Fig. 2(c) shows the middle spine dynamics during turning behavior, which corresponds to the motor pattern.
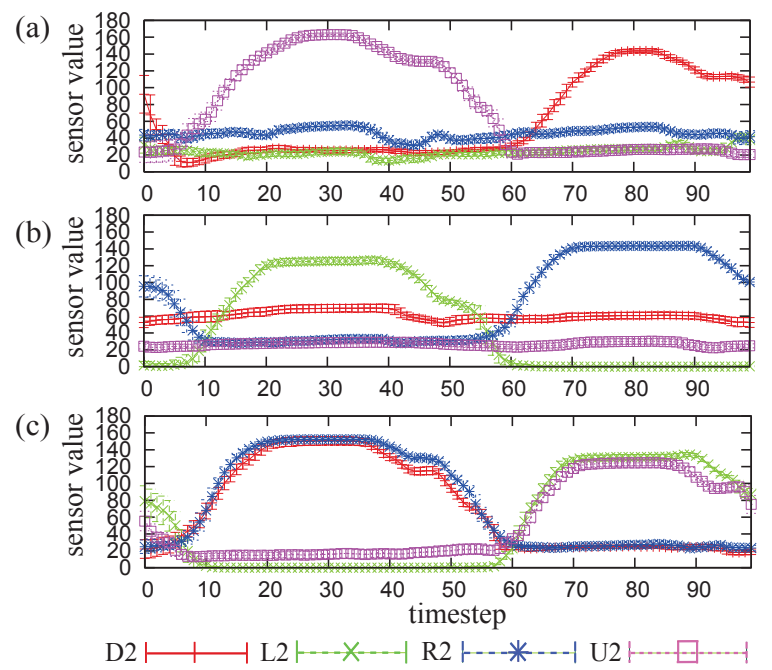

Fig. 2. Middle spine dynamics based on SM1 during one cycle in the experiments of (a) bounding, (b) trotting, and (c) turning right. Responses of the sensory value of 36 cycles of oscillatory motor commands are used to obtain the average. The error bars represent the standard deviation.
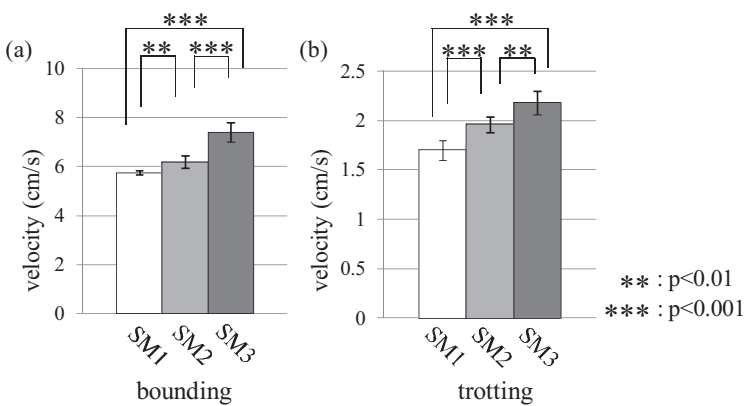

Fig. 4. Comparisons of the averaged velocity for SM1, SM2, and SM3 for the bounding gait (a) and the trotting gait (b). For both (a) and (b), the error bars show the standard deviation. In (a), the averaged velocity for SM1, SM2, and SM3 are 5.76 $\pm 0.09,6.20 \pm 0.26$, and $7.41 \pm 0.40$, respectively. In (b), the averaged velocity for SM1, SM2, and SM3 are 1.70 $\pm 0.1,1.96 \pm 0.08$, and $2.18 \pm 0.12$, respectively. For each plot, asterisks indicate significant differences, $* *: p<0.01$, and $* * *: p<0.001$.

The robot is able to turn at a radius of $0.42 \mathrm{~m}$ with a standard deviation of 0.02 .

\section{B. The effect of spinal morphology on the bounding and} trotting gaits

To capture the internal spine dynamics, we picked force sensors D2 and L2 as typical cases for the bounding and trotting gaits, respectively, due to their sensitiveness to each gait. In both cases, SM1 endures more pressure compared to the rest (Fig. 5), because all the silicon blocks fill in the gap and do not offer much freedom. The absence of some silicon blocks from SM2 and SM3 results in less pressure and a wider space to bend, resulting in a higher speed.

The robot with SM3 shows the best performance and is able to reach a speed of $7.41 \mathrm{~cm} / \mathrm{s}$ with a standard deviation 

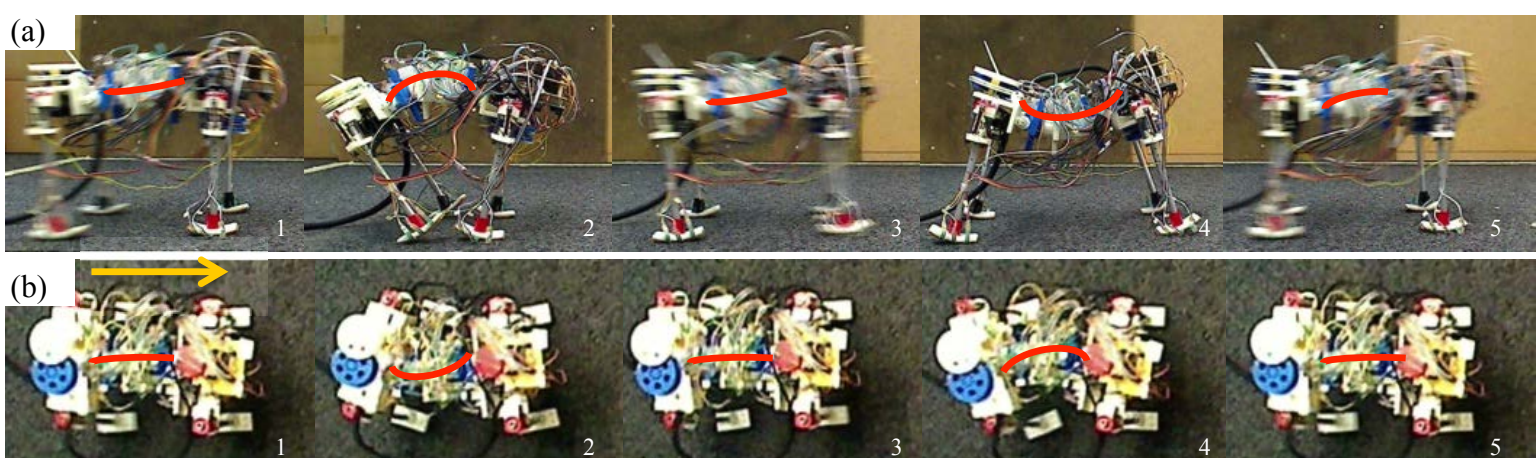

Fig. 3. Sequential pictures of spinal movement in (a) the bounding gait and (b) the trotting gait. Yellow arrow represents the walking direction.
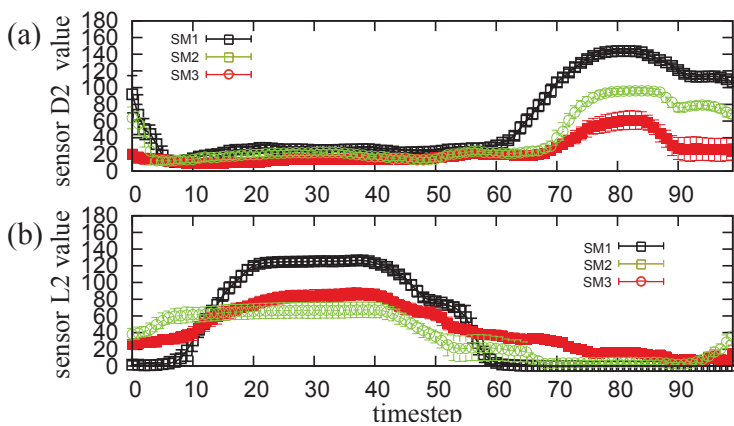

Fig. 5. Comparison of the typical average response curves in the middle spine. (a) Force sensor D2 is taken as the typical case for the bounding gait, and (b) Force sensor L2 is taken as the typical case for the trotting gait. Responses of the sensory value to 36 cycles of oscillatory motor commands are used to obtain the average. The error bars represent the standard deviation.

of 0.4 for the bounding gait and a speed of $2.18 \mathrm{~cm} / \mathrm{s}$ with a standard deviation of 0.12 for the trotting gait (Fig. 4). It is easier for SM3 to pull the back legs forward to increase the stride due to the rear spinal joint. SM2 has more freedom in the front part, which is expected to lift up the front legs [23] to increase stride length. However, the circuit boards are put on the head and increase weight, so the force needed to lift up the body is compensated by the weight of circuit boards. Therefore, no ground clearance can be observed. SM1 is the worst case due to the limited bending space.

\section{Information theoretical analysis on the bounding gait}

As observed in the previous sections, we can obtain various types of locomotion style only by actuating the spine part. In addition, we confirmed that, especially for the bounding and trotting gaits, even if the motor commands are the same, only by changing the morphology of the spine, the velocity of the robot changes significantly. As we explained, a behavior of robots is generated by the coupling between the controller, the body, and the environment. Thus, the observed difference in velocity would be caused by the modulation of these couplings induced by the change of spinal morphology. In this section, by focusing on the bounding gait, we aim to (a)
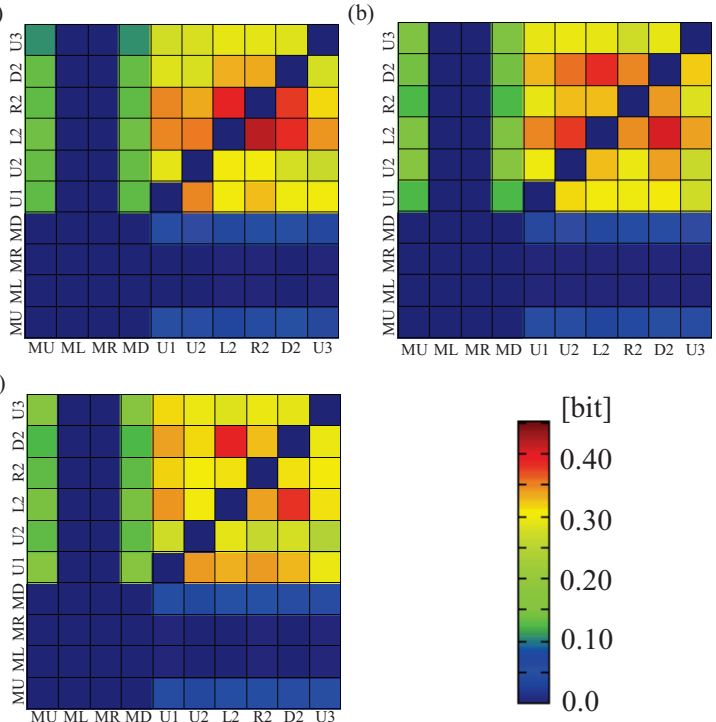

Fig. 6. Comparisons of the averaged value of STE in the bounding gait condition for (a) SM1, (b) SM2, and (c) SM3. For each morphology, the robot is driven in on-the-ground condition for 3 trials with 1200 time steps each. For each STE, the value is averaged over 3 trials. The information transfers from the variables in horizontal axis to vertical axis.

characterize how the change of spinal morphology modulates these couplings by using information theoretic measures.

Fig. 6 shows the averaged STE between the variables in the bounding gait for SM1, SM2, and SM3. Here, in order to make each morphological condition comparable, we selected sensors that remain invariant in each morphology, namely, U1, U2, L2, R2, D2, and U3, and these spinal variables (SP) are used to characterize the spinal dynamics throughout the analysis. As can be seen from Fig. 6, the information transfers from MU and MD to spinal variables, and from spinal variables to spinal variables themselves show remarkably high value in each morphology. These structures are considered to be reflecting all the couplings between the controller, the body, and the environment.

Next, we focus on the relationship only between the con- 
(a)

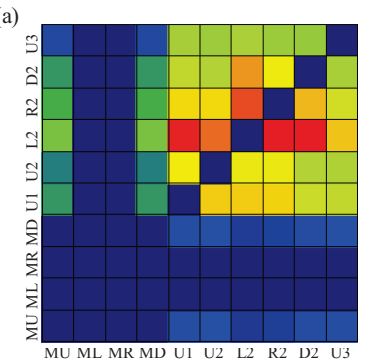

(c)

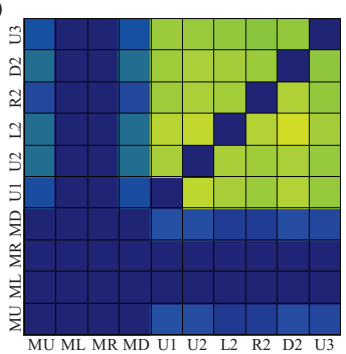

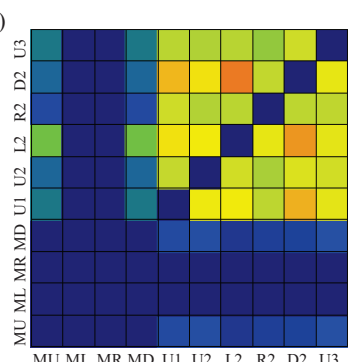

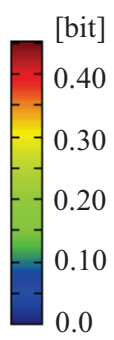

Fig. 7. Comparisons of the averaged value of $S T E_{\text {off }}$ in bounding gait condition for (a) SM1, (b) SM2, and (c) SM3. For each morphology, the robot is driven in off-the-ground condition for 3 trials with 1200 time steps each. For each $S T E_{\text {off }}$, the value is averaged over 3 trials. The information transfers from the variables in horizontal axis to vertical axis.

troller and the body in each morphology. To check this, we run experiments for each morphology by lifting the robot up, so the robot's legs cannot touch the ground (off-the-ground case). Other conditions, such as sensorimotor conditions, are kept the same. Fig. 7 shows the results of the averaged $S T E$ between variables in the off-the-ground case (we call $S T E$ in the off-the-ground case $S T E_{o f f}$ ). As can be seen from Fig. 7, the values seem to be lower than those in Fig. 6 in general. This result suggests that the interaction with the environment is enhancing the information transfer between the variables. In order to confirm this point clearly, we subtracted $S T E_{\text {off }}$ from $S T E$ (we call this $S T E_{\text {subtract }}$ ) and checked how much information is additionally transferred by the interaction with the environment. Fig. 8 shows the results of the averaged value of $S T E_{\text {subtract }}$. We can confirm that, according to the morphological change from SM1, to SM2, and to SM3, there exists a tendency for $S T E_{\text {subtract }}$ to increase.

We checked this increase of information transfer further by summing up the information transfer from M to SP and from SP to SP, and subtracting the corresponding $S T E_{o f f}$, which are respectively denoted as $S T E_{\text {subtract,total }}(M \rightarrow S P)$ and $S T E_{\text {subtract,total }}(S P \rightarrow S P)$, and expressed as follows:

$$
\begin{aligned}
S T E_{\text {subtract }, \text { total }}(M \rightarrow S P)= & \sum_{i=1}^{4} \sum_{j=1}^{6}\left(\operatorname{STE}\left(M_{i} \rightarrow S P_{j}\right)\right. \\
& \left.-S T E_{o f f}\left(M_{i} \rightarrow S P_{j}\right)\right), \\
S T E_{\text {subtract,total }}(S P \rightarrow S P)= & \sum_{i=1}^{6} \sum_{j=1}^{6}\left(S T E\left(S P_{i} \rightarrow S P_{j}\right)\right. \\
& \left.-S T E_{o f f}\left(S P_{i} \rightarrow S P_{j}\right)\right),
\end{aligned}
$$

(a)
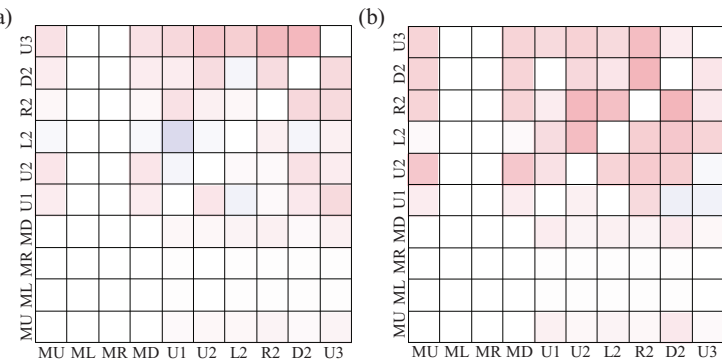

(c)

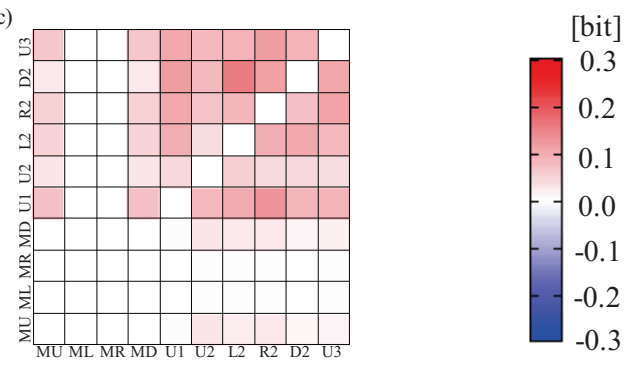

Fig. 8. Comparisons of the averaged value of $S T E_{\text {subtract }}$ in the bounding gait condition for (a) SM1, (b) SM2, and (c) SM3. In order to calculate $S T E_{\text {subtract }}$, we first calculated $S T E_{\text {off }}$ by using 3600 time steps in the offthe-ground condition for each morphology. Then, we subtracted this $S T E_{\text {off }}$ from $S T E$ in each trial and obtained the averaged $S T E_{\text {subtract }}$. Each plot shows the subtracted information transfer from the variables in horizontal axis to vertical axis.

where $M_{1 \sim 4}$ correspond to MU, ML, MR, and MD, respectively. Also, $S P_{1 \sim 6}$ correspond to U1, U2, L2, R2, D2, and $\mathrm{U} 3$, respectively. In addition, we calculated the increase of associations between the variables by summing up the subtracted $P M I$ in a similar manner with $S T E_{\text {subtract,total }}$ between $\mathrm{M}$ and SP $\left(P M I_{\text {subtract,total }}(M, S P)\right)$ and between SP and SP $\left(P M I_{\text {subtract,total }}(S P, S P)\right)$. Note that, since $P M I$ has no directionality, we avoided summing up the case of the same pair of the same variables.

Comparisons of the averaged values of each measure are shown in Fig. 9. We can confirm that the degree of increase of the information transfer and the associations between the variables tends to be enhanced according to the change of spinal morphology in the order of SM1, SM2, and SM3. Interestingly, this result seems to be corresponding to the change of velocity according to the morphological change. This may suggest that the enhancement of the information transfer and associations brought about by the interaction between the environment in the relation around $\mathrm{M}$ and SP leads to the increase of velocity.

\section{CONCLUSION AND DISCUSSIONS}

We demonstrated versatile spine-driven behaviors through embodied coupling between the controller, the body, and the environment. This spine plays a dominant role in generating movement and transferring energy to the legs, which are able to achieve versatile behaviors. This quadruped robot exhibits pronounced flexion-extension and lateral spinal movements, which result in the bounding and the trotting gaits, re- 

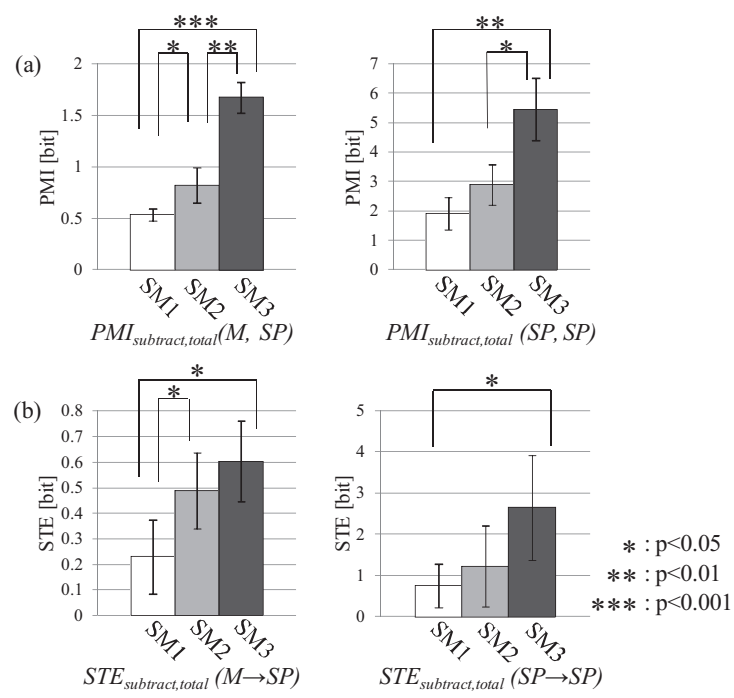

Fig. 9. (a) Comparisons of $P M I_{\text {subtract,total }}(M, S P)$ (left) and $P M I_{\text {subtract total }}(S P, S P)$ (right) according to the morphological changes of the spine. The averaged values of $P M I_{\text {subtract,total }}(M, S P)$ in SM1, SM2, and SM3 are $0.54 \pm 0.06,0.82 \pm 0.17$, and $1.67 \pm 0.15$, respectively. The averaged values of $P M I_{\text {subtract,total }}(S P, S P)$ in SM1, SM2, and SM3 are $1.91 \pm 0.55,2.89 \pm 0.69$, and $5.46 \pm 1.06$, respectively. (b) Comparisons of $\operatorname{STE}_{\text {subtract total }}(M \rightarrow S P)$ (left) and $S T E_{\text {subtract,total }}(S P \rightarrow S P)$ (right) according to the morphological changes of the spine. The averaged values of $S T E_{\text {subtract,total }}(M \rightarrow S P)$ in SM1, SM2, and SM3 are $0.23 \pm 0.14$, $0.49 \pm 0.15$, and $0.60 \pm 0.16$, respectively. The averaged values of $S T E_{\text {subtract.total }}(S P \rightarrow S P)$ in SM1, SM2, and SM3 are $0.75 \pm 0.53,1.23 \pm$ 0.98 , and $2.65 \pm 1.27$, respectively. In order to calculate the averaged value of each measures, we used the same procedure of subtractions explained in Fig. 8. For all the plots, the error bars show the standard deviation, and asterisks indicate significant difference, $*: p<0.05, * *: p<0.01$, and $* * *$ : $p<0.001$.

spectively. Turning behavior can also be realized by the combination of the bounding and trotting gaits. More spinal morphologies have been explored in terms of the VSJ based on the bounding and trotting gaits. Experimental results showed that the speed of SM3 and SM2 is greater than SM1 due to the freedom offered by the absence of some silicon blocks in the spine. SM3 with a rear VSJ showed the best performance in both cases in terms of speed, because a rear VSJ helps rear part of body to gain more freedom to bend, pull the rear legs forward, thus leading to a longer stride. However, there is a trade-off between stability and speed [23]. If more silicon blocks are removed, then more freedom and greater bending angles can be achieved. This results in less stability. In addition, we observed that the robot's performance is better if the stiffness in the downside of the spine is lower than the one in the upside. This finding is consistent with simulation studies which state that the spine stiffness in extension is greater than that in flexion [35].

As we all know, in the biological system, a spine consists of multiple segments, which makes it difficult to model and analyze. However, our study suggests that information theoretic measures might be useful to quantify how the spine affects locomotion through the interaction with the environment. By using the information theoretic analysis, we characterized the information structure of the sensorimotor variables in the bounding gait with different spinal morphologies. As a result, we show that the information transfers and the associations between the variables brought about by the interaction with the environment tend to increase according to the change of spinal morphology. This enhancement of the information structure seems to have a correspondence with the change of velocity. This correspondence should be further investigated in future work.

In this study, the simplest legs without actuation were introduced to investigate spine-driven locomotion. We observed that the legs slide on the ground most of the time due to the lack of ground clearance. This problem can be solved by adding leg actuation, as explained by Gracovetsky, who states that the role of legs is to achieve ground clearance and overcome obstacles, whereas the function of the spine is to generate main force and movement for locomotion [7]. In future, new actuated legs will be designed and applied to this robot to increase dynamical performance. We will also install touch sensors and joint sensors to the legs to sense their internal dynamics to gain a better understanding of the underlying mechanism of the function of the spine in locomotion, e.g., how the force propagates from the spine to the legs based on the information theoretical method.

\section{REFERENCES}

[1] R. M. Alexander, Principles of Animal Locomotion. Princeton University Press, 2002.

[2] I. Poulakakis, J. A. Smith, and M. Buehler, "Modeling and experiments of untethered quadrupedal running with a bounding gait: The Scout II robot," Int. J. Rob. Res., vol. 24, pp. 239-256, April 2005.

[3] J. Buchli and A. J. Ijspeert, "Self-organized adaptive legged locomotion in a compliant quadruped robot," Autonomous Robots, vol. 25, no. 4, pp. 331-347, 2008.

[4] Y. Fukuoka, H. Katabuchi, and H. Kimura, "Dynamic locomotion of quadrupeds Tekken $3 \& 4$ using simple navigation," Int. J. Rob. Res., vol. 24, pp. 239-256, April 2005.

[5] K. Byl and R. Tedrake, "Dynamically diverse legged locomotion for rough terrain," in 2009 IEEE International Conference on Robotics and Automation (ICRA), May 2009, pp. 1607-1608.

[6] S. Gracovetsky, Journal of Biomedical Engineering, vol. 7, no. 3, pp. 205-216, 1985.

[7] S. Gracovetsky and S. Iacono, "Energy transfers in the spinal engine," Journal of Biomedical Engineering, vol. 9, no. 2, pp. 99-114, 1987.

[8] S. Gracovetsky, The Spinal Engine. Springer, cop, 1989.

[9] B. M. Boszczyk, A. A. Boszczyk, and P. Reinhard, "Comparative and functional anatomy of the mammalian lumbar spine," The Anatomical Record, vol. 264, no. 2, pp. 157-168, 2001.

[10] M. A. Lewis and G. A. Bekey, "Gait adaptation in a quadruped robot," Autonomous Robots, vol. 12, pp. 301-312, 2002.

[11] K. F. Leeser, "Locomtotion experiments on a planar quadruped robot with articulated back spine," Master's thesis, Massachusetts Institute of Technology, 1996.

[12] U. Culha and U. Saranli, "Quadrupedal bounding with an actuated spinal joint," in 2011 IEEE International Conference on Robotics and Automation (ICRA), May 2011, pp. 1392-1397.

[13] M. H. H. Kani, M. Derafshian, H. J. Bidgoly, and M. N. Ahmadabadi, "Effect of flexible spine on stability of a passive quadruped robot: Experimental results," in 2011 IEEE International Conference on Robotics and Biomimetics (Robio), Dec. 2011, pp. 2793-2797.

[14] R. Pfeifer, M. Lungarella, and F. Iida, "Self-organization, embodiment, and biologically inspired robotics," Science, vol. 318, no. 5853, pp. 1088-1093, 2007.

[15] R. Pfeifer and J. Bongard, How the Body Shapes the Way We Think: A New View of Intelligence. The MIT Press, 2006. 
[16] K. Nakajima, T. Li, H. Sumioka, M. Cianchetti, and R. Pfeifer, "Information theoretic analysis on a soft robotic arm inspired by the octopus," in 2011 IEEE International Conference on Robotics and Biomimetics (Robio), Dec. 2011, pp. 110-117.

[17] H. Sumioka, Y. Yoshikawa, and M. Asada, "Reproducing interaction contingency toward open-ended development of social actions: Case study on joint attention," Autonomous Mental Development, IEEE Transactions on, vol. 2, no. 1, pp. 40-50, March 2010.

[18] Y. Yamada, S. Nishikawa, K. Shida, R. Niiyama, and Y. Kuniyoshi, "Neural-body coupling for emergent locomotion: A musculoskeletal quadruped robot with spinobulbar model," in 2011 IEEE/RSJ International Conference on Intelligent Robots and Systems (IROS), Sept. 2011, pp. 1499-1506

[19] M. Lungarella and O. Sporns, "Mapping information flow in sensorimotor networks," PLoS Computational Biology, vol. 2 (10), pp. 13011312, 2006.

[20] M. Lungarella, T. Pegors, D. Bulwinkle, and O. Sporns, "Methods for quantifying the informational structure of sensory and motor data,' Neuroinformatics, vol. 3, pp. 243-262, 2005.

[21] N. Schmidt, M. Hoffmann, K. Nakajima, and R. Pfeifer, "Bootstrapping perception using information theory: Case studies in a quadruped robot running on different grounds," Advances in Complex Systems, 2012 (acctped).

[22] Q. Zhao, H. Sumioka, and R. Pfeifer, "The effect of morphology on the spinal engine driven locomotion in a quadruped robot," in The 5th International Symposium on Adaptive Motion of Animals and Machines (AMAM2011), Oct. 2011, pp. 51-52.

[23] Q. Zhao and H. Sumioka, "The effect of robot morphology on locomotion from the perspective of spinal engine in a quadruped robot," in International Conference on Morphological Computation, Sep. 2011, pp. 130-132.

[24] [Online]. Available: http://www.espine.com/anatomy-normal.htm

[25] R. M. Alexander and A. S. Jayes, "Estimates of the bending moments exerted by the lumbar and abdominal muscles of some mammals," Journal of Zoology, vol. 194, no. 3, pp. 291-304, 1981.

[26] [Online]. Available: www.arduino.cc

[27] C. Bandt and B. Pompe, "Permutation entropy: a natural complexity measure for time series," Physical Review Letters, vol. 88, p. 174102 , 2002.

[28] J. M. Amigo, M. B. Kennel, and L. Kocarev, "The permutation rate equals the metric entropy rate for ergodic information sources and ergodic dynamical systems," Physica D, vol. 2010, pp. 77-95, 2005.

[29] T. Haruna and K. Nakajima, "Permutation complexity via duality between values and orderings," Physica D, vol. 240, pp. 1370-1377, 2011.

[30] T. M. Cover and J. A. Thomas, Elements of Information Theory. New York: John Wiley Sons, 1991.

[31] T. Schreiber, "Measuring information transfer," Physical Review Letters, vol. 85, pp. 461-464, 2000.

[32] M. Staniek and K. Lehnertz, "Symbolic transfer entropy," Physical Review Letters, vol. 100, p. 158101(4), 2008.

[33] T. Haruna and K. Nakajima, "Symbolic transfer entropy rate is equal to transfer entropy rate for bivariate finite-alphabet stationary ergodic markov processes," arXiv:1112.2493v2, 2011.

[34] — - "Permutation complexity and coupling measures in hidden markov models," arXiv:1204.1821v2, 2012.

[35] Q. Deng, S. Wang, W. Xu, J. Mo, and Q. Liang, "Quasi passive bounding of a quadruped model with articulated spine," Mechanism and Machine Theory, vol. 52, no. 0, pp. 232 - 242, 2012. 



\section{Appendix C}

\section{Spine as an Engine: Effect of Spine Morphology on Spine-Driven Quadruped Locomotion}

Reprinted from:

Zhao, Q., Sumioka, H., Nakajima, K., Yu, X., and Pfeifer, R.(2014). Spine as an Engine: Effect of Spine Morphology on Spine-Driven Quadruped Locomotion, Advanced Robotics, 28(6): pp. 367-378.

This is the final accepted version. Final version of the article can be found at http:/ / www.tandfonline. com/doi/pdf/10.1080/01691864.2013.867287. 


\title{
Spine as an Engine: Effect of Spine Morphology on Spine-Driven Quadruped Locomotion
}

\author{
Qian Zhao ${ }^{a *}$, Hidenobu Sumioka ${ }^{b}$, Kohei Nakajima ${ }^{a c}$, Xiaoxiang $\mathrm{Yu}^{c}$, and Rolf Pfeifer $^{a}$ \\ ${ }^{a}$ Department of Informatics, University of Zurich, 8050 Zurich, Switzerland; ${ }^{b}$ Advanced \\ telecommunications research institute international,Japan; ${ }^{c}$ Bio-Inspired Robotics Lab, Swiss Federal \\ Institute of Technology Zurich, 8092 Zurich, Switzerland
}

( $v 1.0$ released January 2013)

\begin{abstract}
In quadruped animals, spinal movements contribute to locomotion in terms of controlling body posture and integrating limb and trunk actions. In this paper, we develop quadruped models with different numbers of spinal joints to demonstrate the spine-driven locomotion. Actuated spinal joint(s) are exclusively employed to these models with a minimalistic control strategy. We choose some typical individuals from two models and analyze them on gait properties. Results show that employing the spine morphology with two joints can greatly enhance the stability and speed of locomotion by readjusting center of mass, increasing the stride length, and generating double flight phases similar to running cheetahs gait, which makes significant difference in the speed and the gait. Furthermore, we explore and compare models with more spinal joints. Results show that all gaits emerged from them can be categorized into three types (bounding, bounding with double flight phase, and stotting gaits). However stotting gait may not contribute to locomotion. Instead, it could play a role in body language. Overall, bounding gait with double flight phases is a more biologically inspired locomotive behavior regarding speed and occupancy ratio; model with two spinal joints could be sufficient to emulate biological spine-driven locomotive behaviors.
\end{abstract}

Keywords: spinal engine; spine-driven locomotion; spine morphology; embodiment

\section{Introduction}

Over the past decades, it has been widely accepted that locomotion is generally achieved by the coordination of the legs and the spine is only considered to be carried along in a more or less passive way [1],[2]. This popular hypothesis has been accepted by most of robotics researchers as well as biologists. A considerable amount of research has been conducted on legged robots with little consideration on their spines. Most of the existing quadruped robots are very similar in their morphology, and feature a single rigid body with four legs with individually actuated hips and/or knees [3-6]. They can perform well in some terrains, but they need precise calculation at every time step and therefore lead to high computational load. Furthermore, the resulting locomotive behavior is still much more constrained than its natural counterpart in terms of speed, energy efficiency, maneuverability, and adaptivity to rough terrain.

If we look back to the nature, we find that one of the major differences between these robots and animals is the spine. Such a spine is central to control body posture, provide the foundation to produce the leg's movement, and integrate limb and trunk actions [7]. From the evolutionary point of view, it is natural to consider the spine as an propulsive engine of the vertebrate body to maintain a central role in locomotion in all craniates. Specifically, the anatomy of the spine, and its associated functions and resultant postures are evolved to adapt to the new environment, e.g., 
new function of the spine to stabilize the trunk emerges. Nevertheless, the locomotor function of the spine has never been replaced over time and it is still prevailing in locomotion [7]. Based on mathematical simulations and analysis, Gracovetsky has proposed an alternative biological hypotheses called "Spinal Engine", which emphasizes the role of the spine in human locomotion, i.e., locomotion is firstly achieved by the motion of the spine; the limbs came after, as an improvement but not a substitute [8]. Then, he extended this hypothesis to quadruped animals featuring flexion-extension spinal movement [9], [10]. All of them imply that the spine is crucial to locomotion and such a spine might plays a dominant role in locomotion.

Recently, some researchers have come to realize the important role the spine plays in locomotion, but most only focused on the controller of the spinal joint, and barely paid attention to its morphology [11-13]. All the aforementioned studies simply introduced a spinal joint connecting the fore and rear part without studying further on its morphological parameters and anatomical structure. The concept of embodiment suggests that a system's behavior is generated through the interaction between controller, body (morphology) and environment [14], [15]. In this context, morphology should be regarded as one of important priorities to be studied. So far there are some studies with the focus on the spine morphology. For example, a pneumatically driven quadruped robot has been developed to investigate the stiffness distribution and arrangement of spine muscles in bounding gait [16]. In addition, a biologically inspired spine has been developed and applied to a quadruped robot named Kitty to study the effect of the shape of the spinal column on locomotion [17]. Furthermore, the position of the spinal joint is also studied in Kitty robot [18]. A musculoskeletal quadruped robot has been developed to investigate the stability of locomotion by changing the stiffness at the trunk in crawl, trot, and pace patterns [19]. However, if we look back to the anatomical structure of a biological spine, we find that there still exists a lot of room to study spinal morphological parameters on locomotion, in addition to the features discussed above, to gain a deep understanding of the locomotive function of the spine and how such a function is affected by morphology.

This paper focuses on investigating the role of the spine and the effect of the number of spinal joints in locomotion. First we introduce two models differing in the number of spinal joints to demonstrate the spine-driven locomotive behavior. One model has one spinal lumbosacral joint and the other one has lumbosacral joint and thoracic joint together. Second, four typical individuals from these two spinal morphologies are selected and compared regarding the gait, the attack angle, the ground clearance (GC), and the movement of the center of mass (CoM). The simulation results show that the locomotion can be greatly enhanced by employing the second model with two joints in terms of the stability and speed. Furthermore, the effect of multiple spinal joints on spine-driven locomotion is investigated. The spine morphology varies from three spinal joints to twelve spinal joints. Each morphology is analyzed in detail in terms of gait, speed, success ratio, occupancy ratio. Results show two spinal joints might be a feasible model to emulate the biological spine-driven locomotive behaviors.

\section{Design}

In this section, we describe the design of the models and spinal morphologies and explain our motivation to propose such designs. Next, the selection of the morphological parameters and the design of controller are presented.

\section{1 robot design}

In this model, the fore and rear legs are fixed to the ends of the spine and have no relative rotation with respect to it. In other words, the robot is only actuated by the spine, and leg actuation is not taken into account. Our aim is to test whether or not the spine is the main power to drive the robot, inspired by the biological concept "Spinal Engine" [10], so this design is straightforward 


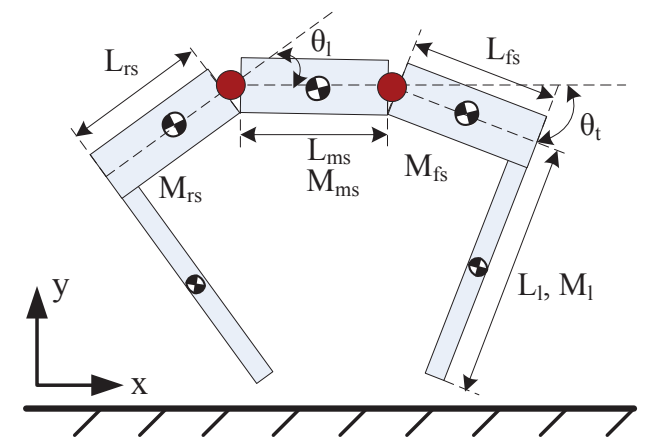

Figure 1. Planar quadruped model of $M 2$. Red solid dots stand for the actuated spinal joints. The specifications of the model are shown in Table 1.

Table 1. Morphological parameters for $M 2$

\begin{tabular}{lcccccccc}
\hline Parameter & $\mathrm{L}_{l}$ & $\mathrm{~L}_{r s}$ & $\mathrm{~L}_{m s}$ & $\mathrm{~L}_{f s}$ & $\mathrm{M}_{l}$ & $\mathrm{M}_{r s}$ & $\mathrm{M}_{m s}$ & $\mathrm{M}_{f s}$ \\
\hline Value & $0.83 \mathrm{~m}$ & $0.33 \mathrm{~m}$ & $0.33 \mathrm{~m}$ & $0.33 \mathrm{~m}$ & $5 \mathrm{~kg}$ & $6.7 \mathrm{~kg}$ & $6.7 \mathrm{~kg}$ & $6.7 \mathrm{~kg}$ \\
\hline
\end{tabular}

L: length; M: weight.

$l$ : leg; $r s$ : rear spinal segment; $m s$ : middle spinal segment; $f s$ : fore spinal segment.

to study the function of the spine and the effect of spine morphology on locomotion.

\section{$2.2 \quad$ Spinal morphology design}

A biological spine is made up of small bones, known as vertebrae, that are stacked on top of each other to create the spinal column. The number of vertebrae varies with the species of the animals from ten in frogs to fifty six in tigers. All of the spinal movements are distributed over the connecting joints of these vertebrae.

We know that the spinal column consists of lumbosacral spine, thoracic spine, and cervical spine [10]. Hence we employed a spinal joint with one degree of freedom (DoF) to emulate the movement of each part. Because the head's movement has less effect on locomotion, we ignored cervical spine in this study. We utilized a lumbosacral joint (LJ) to mimic the role of lumbosacral spine in locomotion. Similarly, the thoracic joint (TJ) is taken to emulate the function of thoracic spine.

As a starting point, we only applied LJ into the model to study the role of lumbosacral spine, because biological findings suggest that the rear position of LJ can produce a particularly marked sagittal displacement of the pelvis [20] [21], which could plays a main role in locomotion. We name this model with LJ morphology one $(M 1) . M 1$ consists of three segments which are a pair of stick-shaped legs, and a spine with a LJ. We simplified this model by taking out the leg actuation, to investigate how the locomotion is achieved by axial driven propulsion and how the spinal morphology affects locomotion.

Because of the existence of a small amount of flexion-extension movement in thoracic spine [22], we added a TJ in the middle between the shoulder and the LJ (Fig. 1) to investigate how it affects locomotion, along with lumbosacral spine. The model with these two joints is named morphology two $(M 2)$. If we fix the movement of TJ, which is $\theta_{t}$ in Fig. 1, and keep the rest parameters of $M 2$, then $M 2$ becomes $M 1$.

We copied some of cheetah's morphological parameters (weights and sizes of the body and legs), and applied them to the models, because a cheetah exhibits noticeable spinal flexion and extension movement when running [23]. Table 1 details morphological parameters we have chosen for $M 2$. 


\subsection{Controller design}

\subsubsection{Minimalistic control strategy}

To focus on the study of spine-driven locomotion and the effect of spine morphology on locomotion, we employed a minimalistic control strategy to this model [24], in which the angular position of the spinal joints is determined by the sinusoidal curve as follows:

$$
\begin{array}{r}
\theta_{l}(t)=A_{l} \sin \left(2 \pi f_{l} t\right)+\psi_{l} \\
\theta_{t}(t)=A_{t} \sin \left(2 \pi f_{t} t+\phi_{t}\right)+\psi_{t}
\end{array}
$$

where $\theta_{l}$ and $\theta_{t}$ indicate the target angular positions of the motors controlling LJ and TJ, respectively. $A, f$ and $\psi$ designate the amplitude, the frequency, and the offset. The phase $\phi$ is the delay between the LJ and TJ. The subscript $l$ and $t$ denote LJ and TJ, respectively. By using this simple control scheme, we are able to evaluate how the morphological properties of the spine can contribute to locomotive behavior. The parameters used in the following experiments are heuristically determined as follows: $f_{t}=f_{l}=1.5 \mathrm{~Hz}, A_{l} \in\left[0^{\circ}, 60^{\circ}\right]$, and $\psi_{l} \in\left[-15^{\circ}, 15^{\circ}\right]$. The rest control parameters $\left(A_{t}, \psi_{t}, \phi_{t}\right)$ will be optimized with Genetic algorithm (GA) described in the following part. Time step $t$ in this paper represents one actuation loop of the control program.

\subsubsection{Genetic algorithm for the sinusoid function controller}

GA [25] is employed to optimize the control parameters $\left(A_{t}, \psi_{t}, \phi_{t}\right)$ for TJ with the attempt to achieve fast and stable locomotive behaviors. The boundaries of these three parameters are decided as follows: $A_{t} \in\left[0^{\circ}, 60^{\circ}\right], \psi_{t} \in\left[-15^{\circ}, 15^{\circ}\right]$, and $\phi_{t} \in\left[-180^{\circ}, 180^{\circ}\right]$. They are encoded as three 8 bit genes and concatenated as a chromosome. Afterwards, a cost function generates an output (the speed of the robot) from this chromosome (control parameters of the robot). Here the cost function is an experiment by which the performance of the robot is evaluated. If the robot is able to move stably and fast, then it is considered as a sucess and the speed is recorded, otherwise, the speed is 0 . We set population size to 600 and the generation number to 10 .

The GA starts with a group of chromosomes known as the population. Natural selection occurs each generation or iteration of the algorithm. After each generation, only the fastest $50 \%$ of the individuals are selected to continue, while the rest are deleted. The next step is to generate a second population of solutions from those selected through genetic operators: crossover and mutation. The probabilities of crossover and mutation are 0.5 and 0.15 , respectively. After the mutations and crossovers take place, the costs (speed) associated with the offspring and mutated chromosomes are calculated. Generation continues to evolve until iteration number exceeds 10.

\section{Experimental Setup}

\subsection{Simulation Setup}

We have implemented models in Mathworks matlab 2009 (64bit), together with the SimMechanics toolbox, which provides a multibody simulation environment. We use blocks provided by SimMechanics to represent bodies, joints, constraints, and actuators. When the model is established, SimMechanics formulates and solves the equations of motion for the complete mechanical system automatically. In this study, motion mode of joint actuation is chosen in which only position, velocity, and acceleration of the angular joints are needed, because we mainly focus on the kinematics of the spine and its resultant locomotive behavior.

In this simulation environment, we constructed a physically realistic interaction model based on a biomechanical study [26]. The vertical ground reaction forces are modeled by one nonlinear visco-elastic element, and the horizontal forces are calculated by a sliding-stiction model. 

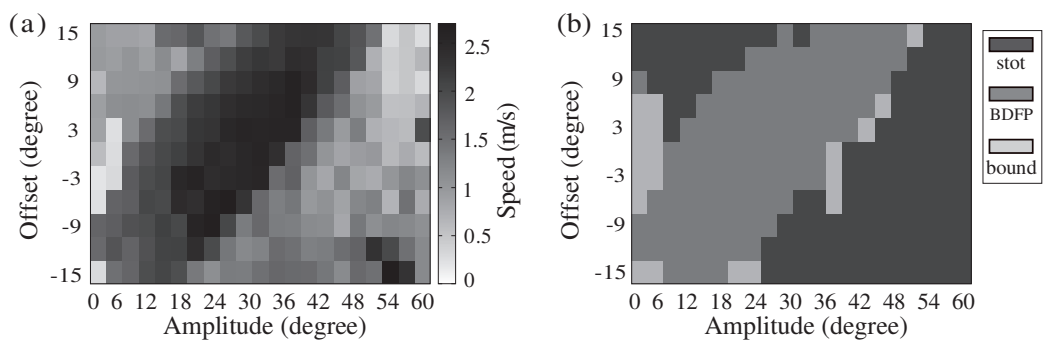

Figure 2. Speed (a) and gait categorization (b) of $M 2 . \mathrm{X}$ axis is amplitude $\left(A_{l}\right)$, and y axis denotes offset $\left(\psi_{l}\right)$ for the LJ.

It switches from stiction to sliding when the velocity of the foot exceeds the specified threshold. We used $0.7,0.8$, and $0.01 \mathrm{~m} / \mathrm{s}$ for the sliding, stiction friction coefficients and the threshold velocity, respectively. Simulations were started from an initial condition with a height of $0.1 \mathrm{~m}$ from a stationary state and run for $50 \mathrm{~s}$.

\subsection{Experimental criterion}

Locomotive behaviors of the planer quadruped model can be categorized into four types: the robot exhibits a stable rapid locomotion; it runs slowly; it exhibits unstable behavior; or it falls over or flies away. Only the first case is considered as meaningful behavior, while the rest are failures. In this paper, we use two methods together to judge the system's stabilizing behavior. Step-to-fall method is used in a way such that the robot is expected not to fall within given time or cycles. Here, we set the time to $50 \mathrm{~s}$. The second one is to calculate the difference between two apex heights of CoM in every two subsequent cycles. If such a difference is less than a threshold, then we assume that this individual is stable, and the speed is recorded. Otherwise, this individual is considered to be a failure and the speed is 0 . This method is simple, but it is efficient to predict the tendency of the stability. For example, if the error between the current cycle and previous cycle exceeds the threshold, but the robot is still able to move, then it suggests a rather high possibility of instable situation for the next cycle where the robot either falls over or flies away.

\section{Results}

In this section, the results of the overall exploration based on two proposed simplified models are presented first, followed by individual analysis in terms of CoM, attack angle, and the gait. Next, the effect of multiple spinal joints ranging from three to twelve on spine-driven locomotion is investigated. Each morphology is analyzed in detail with respect to success ratio, speed, gait, and occupancy ratio.

\subsection{Overall exploration based on two spinal morphologies}

To achieve comprehensive behavioral analysis, we investigated the influence of amplitude $\left(A_{l}\right)$ and offset $\left(\psi_{l}\right)$ on the locomotive behavior for $M 1$ and $M 2$. We varied $A_{l}$ from $0^{\circ}$ to $60^{\circ}$, and $\psi_{l}$ from $-15^{\circ}$ to $15^{\circ}$ with the increment of $3^{\circ}$ in $M 1$. Then we keep the same control parameters for the LJ and optimize the rest three $\left(A_{t}, \psi_{t}, \phi_{t}\right)$ for the TJ in $M 2$.

Figure 2(a) and Figure 4(a) demonstrate that the locomotion is able to be generated by the spinal flexion and extension in $M 2$ and $M 1$. Figure 2(b) shows that there exist three gaits in $M 2$ : bounding (Fig. 3(b)), bounding with double flight phases (BDFP) (Fig. 3(c)) and stotting gaits (Fig. 3(d)), while $M 1$ is only able to generate bounding gait (Fig. 3(a)). The fastest individuals of each gait of $M 1$ and $M 2$ are selected; they are named as $I 1$ in $M 1, I 2$ in bounding in $M 2$, 
(a)

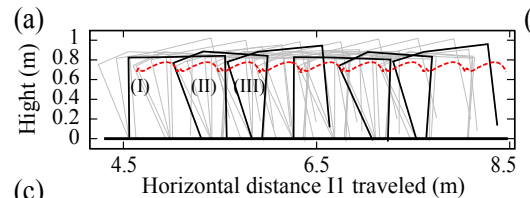

(c)
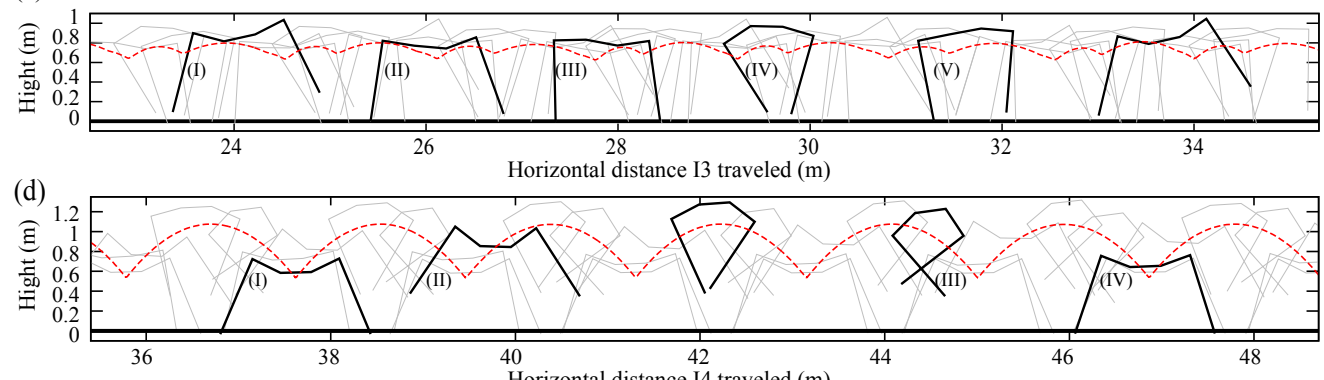

Figure 3. Stick figures illustrating three different behaviors in simulation. The body postures are illustrated every 105 and $735(105 \times 7)$ simulation steps (a), and every 105 and $630(105 \times 6)$ simulation steps (b), (c), (d) (gray and black stick figures, respectively). Red dotted line represent the trajectories of absolute CoM. (a) $I 1\left(A_{l}=18^{\circ}, \psi_{l}=-12^{\circ}\right)$. (b) $I 2\left(A_{l}=36^{\circ}\right.$, $\left.\psi_{l}=-3^{\circ}\right)$. (c) $I 3\left(A_{l}=30^{\circ}, \psi_{l}=-3^{\circ}\right)$. (d) $I 4\left(A_{l}=54^{\circ}, \psi_{l}=-15^{\circ}\right)$

$I 3$ in BDFP in $M 2$, and $I 4$ in stotting in $M 2$, respectively. Since stotting gait exhibits different locomotion pattern where all of the legs touch and leave the ground at the same time, we excluded this gait in the following analysis and will explain it later in section 4.4. Figure 4 shows that $M 2$ can move much faster than $M 1$, and its fastest performance attains $2.6 \mathrm{~m} / \mathrm{s}$, while the fastest one from $M 1$ is $0.59 \mathrm{~m} / \mathrm{s}$.

The attack angle is defined as the angle formed between the leg and the ground in the forward direction when the feet touch on the ground, and the lift up angle is defined in the same way when the feet leave the ground. In both morphologies, greater attack angle of rear legs (Fig. 4(e), (f)) corresponds to faster speed (Fig. 4(a), (b)). With a larger attack angle, the rear legs can rotate the robot's body around the contact point and push it more forward. In addition, less lift up angle of fore legs (Fig. 4(c), (d)), which benefits to propel the body forward, correlates to faster speed (Fig. 4(a), (b)).

In the biological perspective, the CoM moves forward and backward alternatively with respect to its nose during locomotion [27]. Hence, we defined the CoM_S as the distance between the position of CoM and the position of the robot's shoulder, instead of the nose. The range of the CoM_S gets wider, as a result of the increasing amplitude of the bending and extension movement (Fig. 4(e), (f)). Wider range of the CoM_S (Fig. 4(k), (l)) is associated with better performance (Fig. 4(a), (b)), because it offers more freedom to adjust the CoM, benefiting the stabilization of the posture and the enhancement of the speed. Furthermore, with the increase of the speed in $M 1$ and $M 2$ (Fig. 4(a), (b)), the values of the rear and fore boundaries of the CoM_S get smaller (Fig. 4(g)-(j)), which suggests that the horizontal excursion of the CoM moves further to the anterior trunk region.

\subsection{Basic effects of thoracic joint}

To understand the basic effect of LJ in bounding gait and how TJ benefits to the bounding gait, along with LJ, the best individual from $M 1$, namely $I 1$, and $I 2$, the best bounding gait from $M 2$, are chosen and analyzed in detail. The average speed of $I 1$ is $0.59 \mathrm{~m} / \mathrm{s}$ (Fig. $5(\mathrm{c})$ ), while $I 2$ is $1.25 \mathrm{~m} / \mathrm{s}$ (Fig. $5(\mathrm{~d})$ ). Parameters obtained from GA described previously are given in Table 2. 

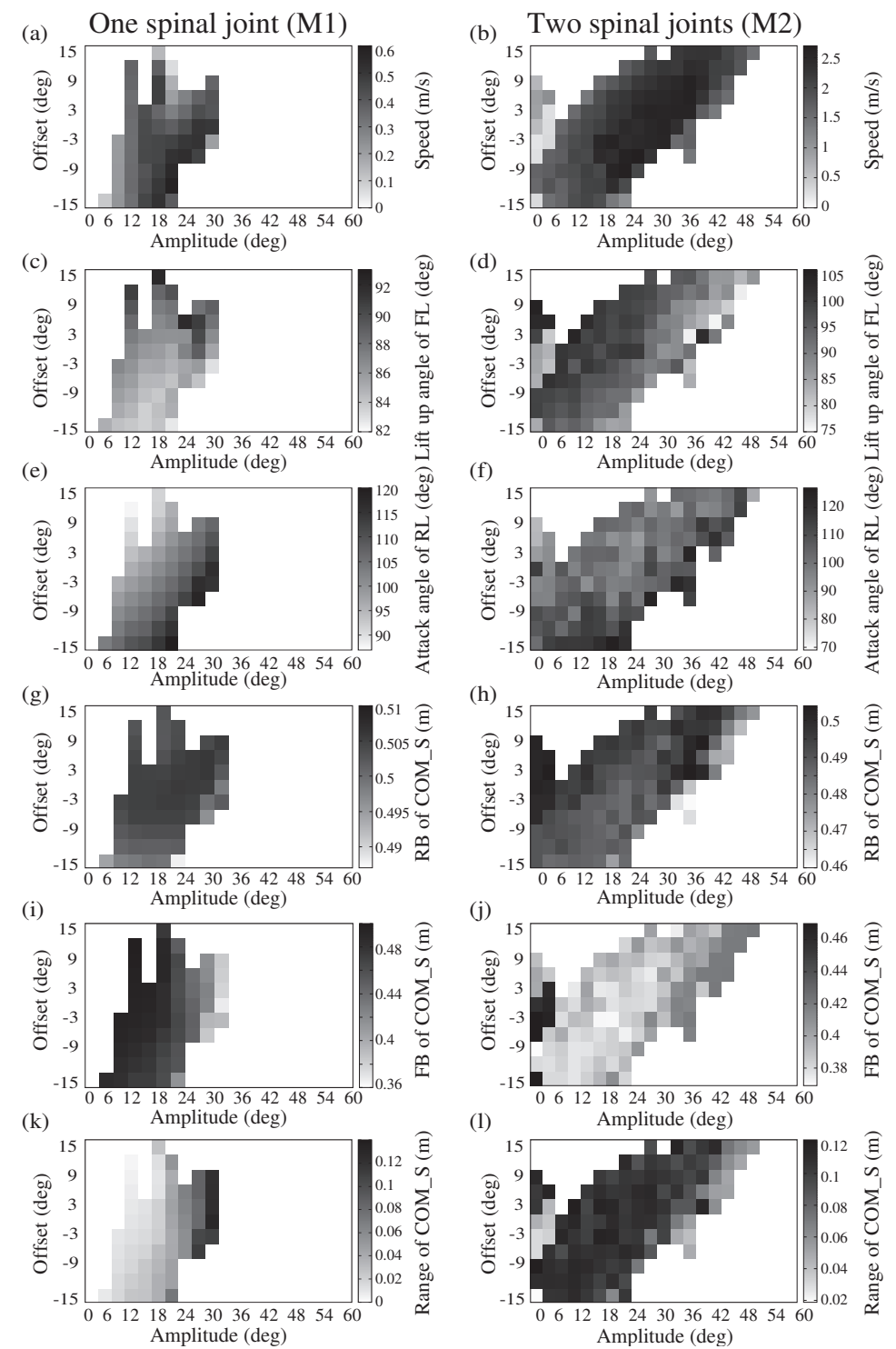

Figure 4. Comparison results of two spinal morphologies ( $M 1$ in the left column and $M 2$ in the right). $\mathrm{X}$ axis is amplitude $\left(A_{l}\right)$, and y axis denotes offset $\left(\psi_{l}\right)$ for the LJ. The intensity of each cell represents the speed in (a), (b); the lift up angle of rear legs (RL) in (c), (d); attack angle of fore legs (FL) in (e), (f); the rear boundary (RB) of CoM_S in (g), (h); the fore boundary (FB) of CoM_S in (i), (j); the range of CoM_S in (k), (l).

\subsubsection{Analysis on spine-driven locomotion}

Figure 3(a), (b) show that the stable locomotive behavior of $I 1$ and $I 2$ can be achieved, even if leg actuation is not taken into account. We observed that four phases exist in $I 1$ (Fig. 5(e)). Since the phase shaded by green has very short duration, low GC of fore legs with $0.016 \mathrm{~m}$, and almost has the same posture as the one after it shaded by blue, we assigned both to phase II. Therefore, $I 1$ is featured three prominent phases as shown in Fig. 3(a). Starting from the original posture (phase I), the spine is flexed and the rear legs are pulled forward until the maximum (phase II). This moves the CoM forward. Afterwards, the spine is extended to allow the lift up 

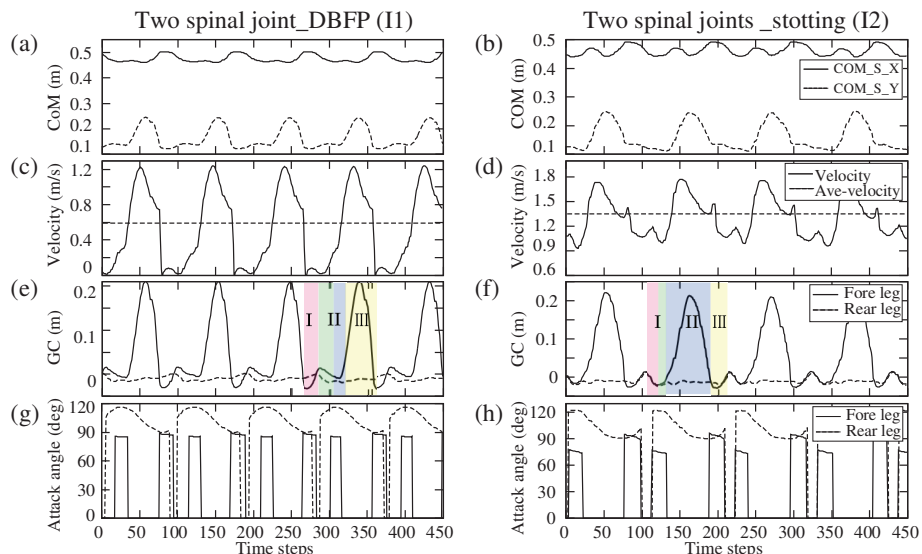

(h)

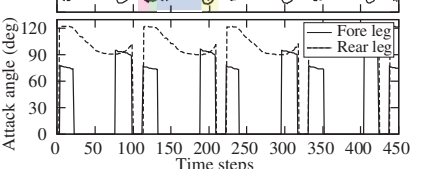

(i)

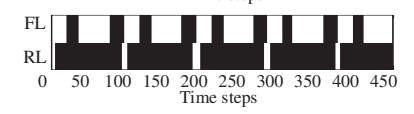

(j)

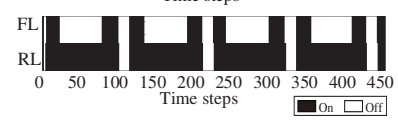

Figure 5. The left and right column are the results of $I 1, I 2$. The $\mathrm{x}$ axis represents time steps. From top to bottom, the $\mathrm{y}$ axis stands for the movement of CoM relative to the shoulder (a),(b), the velocity (c), (d), the height of ground clearance (e), (f) (areas shaded stand for phases, consistent with phases marked in Fig. 3), the attack angle (g), (h), and the footfall pattern (i), (j).

\begin{tabular}{lccccc} 
Table 2. Parameters for $I 1, I 2$ and $I 3$ \\
\hline Parameters & $\mathrm{A}_{l}$ & $\psi_{l}$ & $\mathrm{~A}_{t}$ & $\psi_{t}$ & $\phi_{t}$ \\
\hline$I 1$ & $18^{\circ}$ & $-12^{\circ}$ & $/$ & $/$ & $/$ \\
$I 2$ & $36^{\circ}$ & $-3^{\circ}$ & $19^{\circ}$ & $13^{\circ}$ & -0.67 \\
$I 3$ & $30^{\circ}$ & $-3^{\circ}$ & $24^{\circ}$ & $13^{\circ}$ & -0.214 \\
\hline
\end{tabular}

of the fore legs, leading to the back-moving of the CoM (phase III). In the next step, the fore legs touch the ground, and the CoM moves forward again (back to phase I). The same process repeats.

Similarly as $I 1, I 2$ also has three important phases (Fig. 3(b)). The difference with $I 1$ comes from the further flexed spine caused by combining the flexion of LJ and TJ. This then pulls the rear legs more forward than $I 1$ (phase I) and leads to a higher attack angle of $123^{\circ}$ (Fig. 5(h)), compared to $I 1$ with $116^{\circ}$ (Fig. $5(\mathrm{~g})$ ). The rest of the cycle follows the same procedure as in $I 1$. We categorized $I 1$ and $I 2$ to the bounding gait due to their similar phases and footfall patterns (Fig. 3(a), (b), Fig. 5(i), (j)).

\subsubsection{Ground clearance}

GC for the fore legs is almost the same in $I 1$ and $I 2$ (Fig. 5(e), (f)). It has two peaks: one lower about $0.015 \mathrm{~m}$, and the other one higher about $0.22 \mathrm{~m}$. However, GC for the rear legs is different for $I 1$ and $I 2$. The former has its GC barely noticeable $(0.003 \mathrm{~m})$, while the latter has a much higher GC $(0.01 \mathrm{~m})$. This is due to the inclusion of the flexion of the additional spinal joint $(\mathrm{TJ})$.

\subsubsection{Attack angle}

In these two models, attack angle changes along with the body posture controlled by the spinal controller. Wider range of attack angle of fore legs in $I 2$ enhances locomotion, because it is able to increase the stride length by propelling the body forward further. It varies from $94^{\circ}$ to $74^{\circ}$ in phase I (Fig. 5(h)), as a result of the additional flexion of TJ. Therefore, it can push the body forward further than $I 1$, whose angle is almost constant, $90^{\circ}$ (Fig. 5(g)). In addition, larger attack angle of rear legs contributes to the increase of the stride length by pushing the body more forward. 
Table 3. Results of CoM_S in $I 1, I 2, I 3$

\begin{tabular}{cccc|ccc}
\hline & \multicolumn{2}{c}{ CoM_S $_{\text {horizontal }}(\mathrm{m})$} & \multicolumn{3}{c}{ CoM_S } & \multicolumn{2}{c}{ vertical $_{(\mathrm{m})}$} \\
& Fore $_{b}$ & Rear $_{b}$ & Range & Low $_{b}$ & High $_{b}$ & Range \\
\hline$I 1$ & 0.46 & 0.5 & 0.04 & 0.12 & 0.25 & 0.13 \\
$I 2$ & 0.45 & 0.49 & 0.04 & 0.12 & 0.25 & 0.13 \\
$I 3$ & 0.39 & 0.49 & 0.1 & 0.11 & 0.29 & 0.18 \\
\hline
\end{tabular}
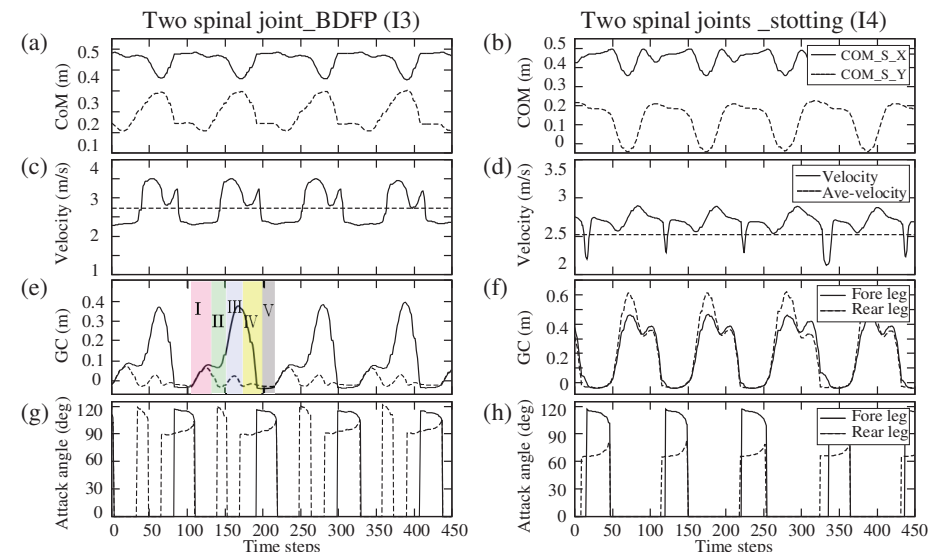

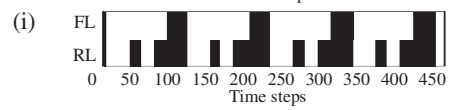

(j)

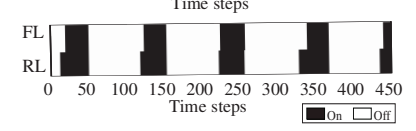

Figure 6. The left and right column are the results of $I 3, I 4$. The $\mathrm{x}$ axis represents time steps. From top to bottom, the $\mathrm{y}$ axis stands for the movement of CoM relative to the shoulder (a),(b), the velocity (c), (d), the height of ground clearance (e), (f) (areas shaded stand for phases, consistent with phases marked in Fig. 3), the attack angle (g), (h), and the footfall pattern (i), (j).

\subsubsection{Movement of the center of mass}

Figure 5(a), (b) show that the horizontal motion of the CoM in the body is only determined by flexion and extension of the spine. This underlines the determinant role of the spine as the main engine for the locomotion. The movement of CoM relative to the shoulder is not constant (Fig. 5(a), (b)). The horizontal excursion of the CoM is in coupling with the motion of the spine. During spinal extension, the CoM moves to the posterior part of the spine, but it moves to the anterior part during spinal flexion. This horizontal excursion equals about $4 \%, 4 \%$ of the body length in $I 1, I 2$, respectively (Table 3 ). The extension phase of the spine is coupled with a upward movement of the CoM. In the flexion phase, after initial ascent, the CoM moves downward (Fig. 5(a), (b)). The excursion of the vertical movement of the CoM is about $13 \%$, $14 \%$ of the body length in $I 1, I 2$, respectively (Table 3 ).

\subsection{Dynamic locomotion induced by double flight phases}

To investigate how BDFP gait is generated and how it contributes to the locomotion, we selected the fastest one in BDFP gait from $M 2$, namely $I 3$, and compared it with $I 1$ and $I 2$. I3 can reach up to $2.75 \mathrm{~m} / \mathrm{s}$ (Fig. $6(\mathrm{c}))$.

\subsubsection{Analysis on spine-driven locomotion}

There exists a high degree of co-ordination between spinal flexion and the placing of the feet on the ground to maximize stride length and increase speed in $I 3$. I3 mainly differs from $I 1$ and $I 2$ in the gait (Fig. 3(a), (b), (c)). It is characterized by five phases, two of which are flight phases, instead of one, in each stride. Figure 3(c) shows that one takes place when the spine is 
at maximal extension (phase I); the other one occurs when the maximal flexion of the spine is achieved (phase IV).

The frequency of $I 3$ is the same as $I 2$ and $I 1$, but the speed is much faster than both, due to longer stride length. This is caused by the double flight phases exclusively showing up in $I 3$.

\subsubsection{Ground clearance}

I3 has pronounced GC not only for fore legs with $0.37 \mathrm{~m}$, but for rear legs (Fig. 6(e)) . It exhibits two flight phases in rear legs in each cycle: one is with GC of $0.073 \mathrm{~m}$ and the other one is of $0.09 \mathrm{~m}$, which are much higher than $I 1$ and $I 2$.

\subsubsection{Attack angle}

For the rear legs, it has similar value of attack angle to $I 2$, but it has a smaller lift up angle of $90^{\circ}$ (Fig. $6(\mathrm{~g})$ ), which can crouch more and push the body forward further, compared to I2 with the angle of $105^{\circ}$ (Fig. $5(\mathrm{~h})$ ).

\subsubsection{Movement of the center of mass}

Figure 6(a) shows that $I 3$ has much wider horizontal and vertical movements of the CoM, compared to $I 1$ and $I 2$ during one cycle (Fig. 5(a), (b)). Table 3 shows the boundaries and the range of the CoM_S in horizontal and vertical direction. This horizontal excursion equals around $10 \%$ and vertical excursion is about $18 \%$ of the body length in I3. We observed that values of fore boundary and rear boundary of the horizontal movement of CoM_S in I2 and I3 are smaller than $I 1$, which suggests that $I 2$ and $I 3$ are able to move the CoM forward more efficiently than $I 1$, benefiting the rapid locomotion. Moreover, the excursion range of the CoM_S in $I 3$ is wider than $I 1$ and $I 2$, offering more freedom to adjust the CoM to stabilize the robot itself.

\subsection{Stotting gait emerged from pronounced spinal flexion and extension movements}

Interestingly, in addition to bounding and $B D F P$ gaits in $M 2$, we found stotting gait where all of the legs land and lift off the ground at the same time (Fig. 3(d)). Figure 2 indicates that when the amplitude $\left(A_{l}\right)$ is lower than $24^{\circ}$, then stotting gait is more likely to happen in the high offset $\left(\psi_{l}\right)$ area where the spine extends more than flexes. It also shows that while amplitude starts to increase from $24^{\circ}$, the range of offset stotting gait starts to spread is getting wider, which suggests that the effect of the difference in magnitude between flexion and extension on the gait gets smaller, even disappears when amplitude is greater than $54^{\circ}$. In a word, this implies that scotting gait has more chance to take place in the high amplitude area where spine exhibits pronounced flexion and extension movements.

To understand how the stotting gait behaves, and how it differs with the other two gaits, we chose the fastest stotting gait from $M 2$, named it as $I 4$, and analyzed its locomotive features, e.g., the movement of CoM, GC, attack angle. The average speed of $I 4$ is $2.5 \mathrm{~m} / \mathrm{s} \mathrm{(Fig.} 6(\mathrm{~d})$ ). Its four legs jump off and on the ground at the same time (Fig. 6(f), (j)). GC of fore legs (0.6 $\mathrm{m})$ is slightly higher than the rear legs $(0.48 \mathrm{~m})$ (Fig. 6(f)).

We observed that there are four prominent phases in one cycle as shown in Fig. 3(d). Starting from the original posture (phase I), the robot jumps off the ground when the spine starts to flex (phase II). Then it achieves maximal spinal flexion in the air (phase III) ${ }^{1}$, which is immediately followed by spinal extension while the robot is still in the air (phase IV). The spine continues to extend until it lands the ground (back to phase I). In this stotting gait, there exists only one flight phase per cycle where the spinal flexion and extension take place.

${ }^{1}$ In this study, we set up a two-dimensional planar model where the other one dimension about the width is not clearly defined. This may provide possibility to study asymmetrical width of the model. In phase III, it seems that fore legs and rear legs are colliding. Actually, we could explain that the fore legs go in between the two rear legs the rear legs, which is commonly observed in animals [28], because the width of shoulders is narrower than that of hips. 


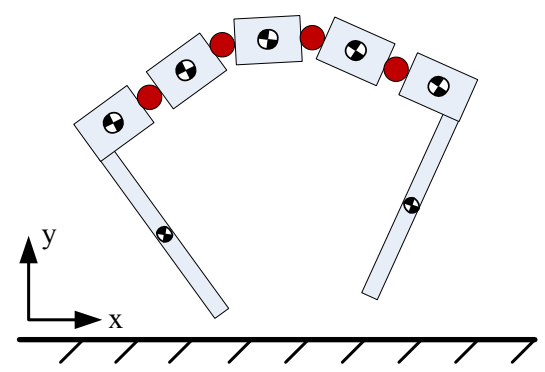

Figure 7. An example of planar quadruped model with multiple spinal joints: $M 4$. Solid red dots represent the actuated spinal joints.

\subsection{Exploration on multiple spinal joints}

To check if this simplified model with two spinal joints $(M 2)$ discussed previously is sufficent to emulate biological spine-driven locomotion, compared to models with multiple spinal joints, we extend experiments by introducing more spinal joints, while keeping most of the setting the same.

\subsubsection{Experiments setting}

Multiple spinal joints are employed and a new model is constructed (Fig. 7), where the number of spinal joints increases from three to twelve. We name each spine morphology by $M i$, where $i$ represents the number of spinal joints. These joints are evenly distritbuted along the spine. The total weight of the spine including all of spine segments and joints is the same as $M 1$ and $M 2$. We start to set the amplitude to $60^{\circ}$ for each morphology. If no successful individuals can be found within the given number of 80,000 , then the amplitude decreases by $10^{\circ}$ until successful cases occur. In the end, the range of the parameters of each morphology is shown in Table 4. Moreover, the choice of those ranges is reasonable, because they are wider than the biological spinal motion range, e.g., cat [29], equine [30], pika [31]. The criterion to evaluate the performance is the same as the one taken in $M 1$ and $M 2$ cases.

\subsubsection{Exploration on more spinal joints}

We calculated the success ratio of each morphology by dividing the number of successful individuals which are able to move forward periodically and stably without falling over by the total number of individuals $(80,000)$. We observed that with the increase of the spinal joints, the success ratio drops exponentially (solid black line in Fig. 8). This is caused by the increase of dimension of controller parameters, e.g., 23 parameters for $M 8$. Therefore, we stopped adding spinal joints when its success ratio is below the threshold (1\%). As a result, M12 is the model with the maximal number of spinal joints.

Figure 9 shows the overall exploration of the number of spinal joints ranging from one to twelve. Interestingly, we found that all of the successful individuals from randomly chosen 80,000 individuals can be categorized into three types: bounding gait, BDFP gait, and stotting gait, which is the same as $M 2$. Generally speaking, $B D F P$ gait outperforms the other two gaits regarding speed over all spine morphologies. BDFP gait (green line and bars in Fig. 9) emerges when the number of spinal joints is great than one, and its average speed and maximal speed keep similar with the increase of the spinal joints. This suggests that BDFP gait is caused by multiple spinal joints. However, the introduction of more than two spinal joints does not contribute too much to the speed of the robot. This implies that $M 2$ is sufficient to generate fast $B D F P$ gait. Stotting gait emerges in the same spine morphologies as $B D F P$ gait. However, the fastest speed of stotting gait (red line and bars in Fig. 9) increased greatly from $M 9$ to $M 12$, while its average speed and standard deviation do not vary much. The performance of bounding gait (blue line and bars in Fig. 9) is greatly improved when $M 2$ is taken, and then it remains.

Occupancy ratio is calculated by the percentage of the number of each gait divided by the 
Table 4. Range of parameters for multiple spinal joints.

\begin{tabular}{cc|cc|cc|cc}
\hline Morphology & Amplitude & Morphology & Amplitude & Morphology & Amplitude & Morphology & Amplitude \\
\hline$M 1$ & {$\left[0^{\circ}: 60^{\circ}\right]$} & $M 4$ & {$\left[0^{\circ}: 40^{\circ}\right]$} & $M 7$ & {$\left[0^{\circ}: 30^{\circ}\right]$} & $M 10$ & {$\left[0^{\circ}: 30^{\circ}\right]$} \\
$M 2$ & {$\left[0^{\circ}: 60^{\circ}\right]$} & $M 5$ & {$\left[0^{\circ}: 40^{\circ}\right]$} & $M 8$ & {$\left[0^{\circ}: 30^{\circ}\right]$} & $M 11$ & {$\left[0^{\circ}: 30^{\circ}\right]$} \\
$M 3$ & {$\left[0^{\circ}: 60^{\circ}\right]$} & $M 6$ & {$\left[0^{\circ}: 30^{\circ}\right]$} & $M 9$ & {$\left[0^{\circ}: 30^{\circ}\right]$} & $M 12$ & {$\left[0^{\circ}: 30^{\circ}\right]$} \\
\hline
\end{tabular}

Note: $\psi \in\left[-15^{\circ}, 15^{\circ}\right], \phi \in\left[-180^{\circ}, 180^{\circ}\right]$, the same as $M 2$

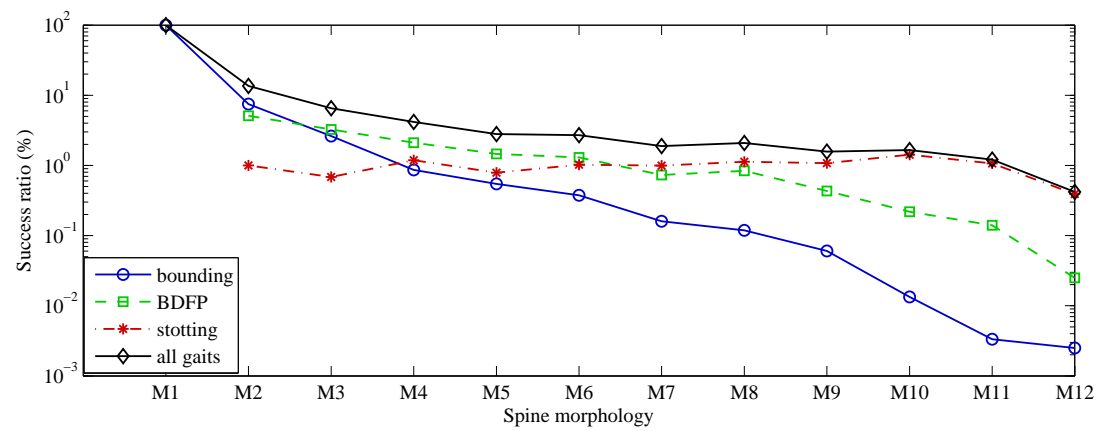

Figure 8. Success ratio of all morphologies changing from one spinal joint to twelve. $\mathrm{X}$ axis stands for the spine morphology ranging from one to twelve spinal joints. The line in blue, green, red, and black are the success ratios of observed bounding gait, $B D F P$ gait, and stotting gait, respectively. Note that y axis is in logarithmic scale.

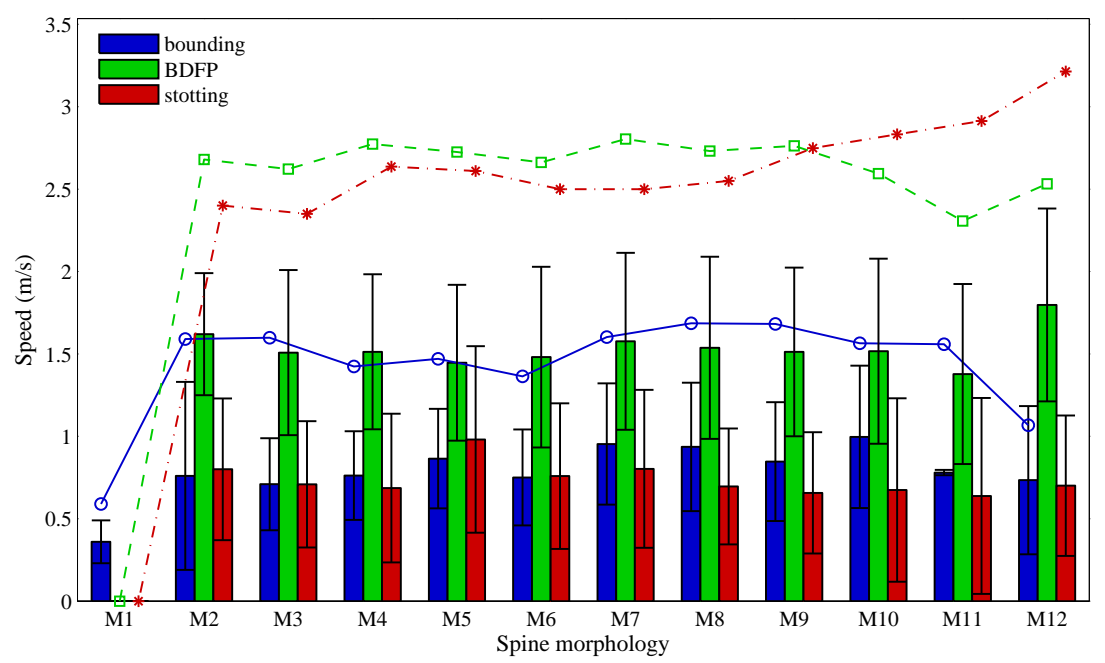

Figure 9. Speed over all spine morphologies. $\mathrm{X}$ axis is the spine morphology ranging from one to twelve spinal joints. $\mathrm{Y}$ axis denotes speed. The average speed, the standard derivation and the fastest speed of each morphology are represented by bars, error bars, and pointed lines, respectively. The results of bounding gait, BDFP gait, and stotting gait are colour coded in blue, green, and red, respectively.

total number of gaits for each spine morphology. Figure 10 shows that the occupancy ratio of bounding gait decreases in the order from $M 1$ to $M 12$, which suggests that this gait is dominant in the low number of spinal joint cases which exhibit less dynamics. If more spinal joints involved in, it is more likely to transform to the other two more dynamical gaits. The occupancy ratio of $B D F P$ starts at $M 2$, and then grows until $M 5$. After $M 5$, it starts to decrease. This gait spreads most of the spine morphologies, except $M 1$. Stotting gait appears from $M 2$ and then goes up greatly. In the end, it dominates in the morphologies with high number of spinal joints. 


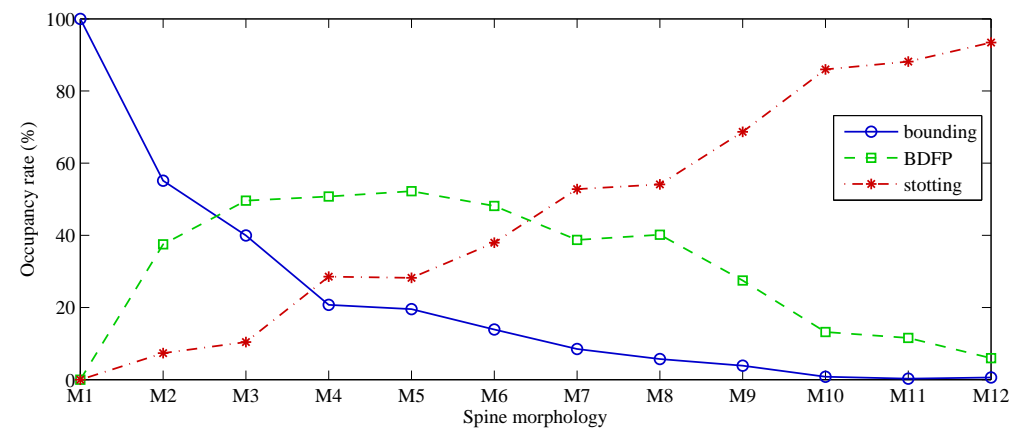

Figure 10. Occupancy ratio of each gait over all spine morphologies. $\mathrm{X}$ axis denotes spine morphology ranging from one to twelve spinal joints.

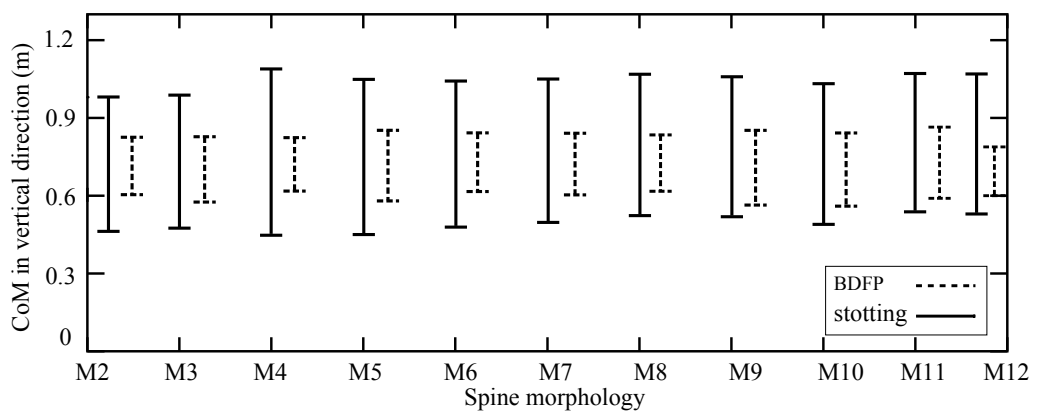

Figure 11. Results of absolute value of $\mathrm{CoM}$ in vertical direction. $\mathrm{X}$ axis denotes spine morphology ranging from two to twelve spinal joints. The range of CoM of $B D F P$ gait in each morphology is represented by dashed line, while the range of CoM of stotting gait is represented by solid line.

This suggests that stotting gait need more DoF to form a specific spine posture in attempt to generate enough power to push itself off the ground.

We recorded the maximum, minimum of absolute CoM in vertical direction of the fastest individuals chosen from $B D F P$ and stotting gaits. Figure 11 shows that the upper and lower bounds of CoM in stotting gait are similar over all spine morphologies. This tendency is similar to $B D F P$ gait. We observed that the maximal value of $\mathrm{CoM}$ in stotting gait is higher than the one in $B D F P$ gait and its minimum is lower than the one in $B D F P$ gait. This results reveal that stotting gait has a wider motion range of CoM in vertical direction, which might be caused by the much longer flight phase. As a consequence, this might result in an increase of energy cost.

\section{Discussion}

$I 2$ is capable of producing more pronounced spinal movements, which contribute to the increase of the stride length by pulling the rear legs forward further than $I 1$, thus increasing the stride length. The attack angle of fore legs of $I 2\left(73^{\circ}\right)$ is less than $I 1\left(85^{\circ}\right)$ when they lift off the ground, caused by additional TJ, which can propel the body forward. We believe that multiple spinal joints are able to provide the body with more freedom to enlarge the swing of the limbs and increase the stride length.

$I 2$ and $I 3$ mainly differ in the speed and the gait, as a result of the amplitude of spinal movements (Table 2). I3 almost runs twice as fast as $I 2$. It reaches the maximal extension and flexion in two flight phases per stride, while $I 2$ is only suspended once in each stride. In 


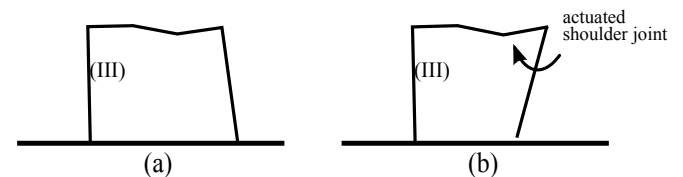

Figure 12. (a) Double stance phase (phase III) in Figure 3, and (b) the possible way to transfer (a) to rear leg stance phase by employing actuated shoulder joints.

addition, the gait of $I 3$ exhibits greater proportion of flight in total stride. These results are in agreement with studies of the motions of the running cheetah and horse [23]. A horse, with relatively rigid spine generating less spinal movements [30], can be represented by $I 2$, and a cheetch, featuring pronounced spinal movements, is suitable to be simplified as I3. We conclude that the double flight phases, together with greater proportion of flight, contribute to its longer stride [23]. However, I3 exhibits an unnecessary stance phase (phase III in Fig. 3(c)), which does not exist in cheetah running. We could eliminate this phase by adding actuated shoulder joints (Fig. 12). Phase III is the transition phase from Phase II when the spine starts to flex to phase IV when the robot exhibits flight phase. In phase III, the flexion starts but it takes time to generate enough force for the fore legs to take off immediately. As a consequence, all of the legs are on the ground in a short moment, resulting in this double stance phase. If actuated shoulder joints are employed in the very beginning of this phase to swing the fore legs backward, then the fore legs might be lifted up. In this case, the double stance phase becomes rear leg stance, and therefore $I 3$ can exhibit a more cheetah-like bounding gait.

The amplitude of the vertical motion relative to the nose is about $13 \%, 13 \%, 18 \%$ of the body length in $I 1, I 2, I 3$, respectively. This is higher than the average value of $10 \%$ observed from human [32] and pika [27] running. The reduction of the vertical displacement of CoM could be achieved by introducing springs in the legs and adjusting their spring-mass systems by increasing the angle swept by the stance legs while keeping leg stiffness nearly constant [33] [34].

Stotting gait emerges when the flexion-extension is pronounced. Such a gait consists of four phases, but there does not exist a stance phase between fully spinal flexion and extension phases (Fig. 3(d)). This behavior can not be found in some common gaits for fast locomotion $[23,35,36]$. Additionally, Alexander [37] states that the function of such a stance phase is to obtain internal kinetic energy and prepare to transform this kinetic energy to potential and external kinetic energy for the next phase. This implies that this stance phase is necessary to coordinate the spinal flexion and extension movements to reach a faster speed. Furthermore, stotting is more dynamic than the rest two gaits, because of its much higher GC and wider excursion of absolute $\mathrm{CoM}$ in vertical direction. Hence these features might lead to slow speed and energy inefficiency. In addition to time and energy cost, stotting could even cause survivorship cost [38]. This is because of the high jumping movements which make the animal visible, and presumably also vulnerable, to the predator. Therefore these studies suggest that stotting is not an optimal gait for fast locomotion especially when the gazelle is chased by a cheetah.

However interestingly, quite similar behaviour can still be observed in some quadruped animals, e.g., dog, gazelles. In the case of gazelles, sometimes, they stot wherein they punch the ground with their stiff limbs, and with a hunched back, propel themselves high in the air in a series of bounds. For a moment between leaps, they float, suspend in mid air. So the question arises: what is the function of stottig; why evolution selects it and still maintains it. Some biological researchers investigated these questions and proposed some reasonable hypotheses [39], [40]. First, stotting appears to inform the predator that it has been detected. As a consequence, cheetahs usually abandon hunts in response to it. Second, neonate informs its mother that it has been disturbed and needs defence. Thirdly, it is also a signal of danger. They use this signal to notice all of the group members that danger is around. In summary, stotting exhibits very noticeable behavior, and plays a role in body language to communicate not only with group members, but also with predators, instead of enhancing locomotion.

We found that the average speed of $B D F P$ generally is faster than that of bounding gait 
and stotting gait over all morphologies ranging from two spinal joints to twelve joints (Fig.9). Moreover, BDFP spreads almost over all of the morphologies, except $M 1$ (Fig.9). Therefore, these two aspects indicate that $B D F P$ gait is more biologically plausible for fast locomotion, compared to stotting and bounding gait. Since $M 2$ with two spinal joints is able to produce such $B D F P$ gait, we believe that $M 2$ is suitable to emulate spine-driven locomotion. The question arises: since two joints are sufficient, why animals evolved much more joints? One possible solution is that more spine joints are easier to form a much smoother curvature of the spine in an attempt to share the load distritbuted along the spine, thus protecting the spine from being damaged [41].

If we look back to nature, we find that most of features of the spine are asymmetrical, for example, different length of lumbosacral spine, thoracic spine. In this study, when the number of spinal joints are greater than one, all of the joints in this morphology have possibilities to rotate within certain degrees or stop moving, because of the setting of the motion range. If one joint or some joints choose to stop rotating, then the spine becomes asymmetrical case. Therefore, our current results are applicable to these asymmetrical cases. However, there still exists a lot of room to study the effect of asymmetrical structures on locomotion, for example, the stiffness distribution of spine muscles, the shape of the spinal column, which are difficult to establish models in the simulation. Therefore, we did these studies in a real quadruped robot called Kitty which features a tendon-driven spine [17], [18].

\section{Conclusion and Outlook}

This novel study has successfully tested the biological concept of "Spinal Engine" and demonstrated spine-driven locomotion in a quadruped robot where legs are not actuated. It suggests that the motion of the spine is a determinant factor in the locomotion. The change of spine posture serves the placement of the CoM relative to the ground contact point, working as an engine to propel the body; limbs might be looked at as servants of the trunk to assist locomotion [10].

$M 2$ performs better than $M 1$ in terms of the speed and stability. $M 2$ is able to produce more freedom to pull the rear legs forward, increase the stride length, and move the CoM more efficiently forward. Therefore the speed is increased. In addition, it benefits stability by using additional TJ to optimize the movement generated by the LJ by readjusting unstable posture or enhancing the extension-flexion movement. I3, the fastest individual from $M 2$, outperforms $I 2$ due to its double flight phases and greater proportion of flight in total stride, as a result of more pronounced spinal movement. This is similar to what we observe from the cheetah's running, which makes significant difference in the speed and gait.

A new gait called stotting emerges when the spinal flexion-extension is enough high. It exhibits much higher GC and wider vertical excursion of CoM. In addition, the stance phase between spinal flexion and extension phase is missing, which has the function to transfer energy and power the spinal extension movements. We think such features lead to energy inefficiency and slow speed. Therefore it is not a suitable model for fast locomotion. Instead, it plays a role in communication, e.g., tell predator that it has been seen, inform mother to get protection, and warn its group members of the danger.

In the experiments of exploring multiple spinal joints, first, we found that the BDFP gait has a faster speed than the bounding and stotting gait over all of the spine morphologies ranging from two to twelve spinal joints. Second, the BDFP gait spreads almost all of the spine morphologies, except $M 1$. Moreover, as discussed before, the $B D F P$ gait, which features double flight phases, is able to emulate cheetah-like locomotive behaviours to a great extent. Therefore all of these evidences support that BDFP gait is a more biologically inspired locomotive behavior. Since $M 2$ is sufficient to produce such a gait, it suggests that $M 2$ could be a feasible model to emulate the biological spine in quadruped locomotion.

In the future, compliant and actuated legs will be introduced to study how to reduce vertical 
excursion of the CoM. In addition, the way of how to coordinate legs and the spine's movements will be investigated as well. More importantly, the introduction of elastic elements to the spine is crucial to get closer to the biological spine model to investigate the energy efficiency, energy transfer, etc. In this case, force control of actuation joint in Matlab simulator will be employed and the methods to tune the force will be studied as well. Further studies of asymmetrical structure of the spine will be another future topic, for instance, the spine muscle distribution, the weight distribution of the spine. Furthermore, a more biologically inspired controller, e.g., central pattern generater, will be taken into account in the future work, with the attempt to better mimic the animals spine locomotive mechanism. In the near future, on-site experiment is necessary to be implemented to validate the correctness of these optimized gaits. Since the results show that two spinal joints are sufficient to represent a biological spine, we will improve the Kitty robot [18] by adding one additional spinal joint to validate the simulated results.

\section{References}

[1] Alexander RM. Principles of animal locomotion. Princeton University Press. 2002.

[2] Raibert M. legged robots that balance. The MIT Press. 2000.

[3] Poulakakis I, Smith JA, Buehler M. Modeling and experiments of untethered quadrupedal running with a bounding gait: The scout ii robot. The International Journal of Robotics Research. 2005; 24(4):239-256.

[4] Buchli J, Ijspeert AJ. Self-organized adaptive legged locomotion in a compliant quadruped robot. Autonomous Robots. 2008;25(4):331-347.

[5] Fukuoka Y, Katabuchi H, Kimura H. Dynamic locomotion of quadrupeds Tekken $3 \& 4$ using simple navigation. Int J Rob Res. 2005 April;24:239-256.

[6] Byl K, Tedrake R. Dynamically diverse legged locomotion for rough terrain. In: 2009 ieee international conference on robotics and automation (icra). 2009 May. p. 1607-1608.

[7] Schilling N, Carrier DR. Function of the epaxial muscles in walking, trotting and galloping dogs: implications for the evolution of epaxial muscle function in tetrapods. The Journal of Experimental Biology. 2010;213(9):1490-1502.

[8] Gracovetsky S. An hypothesis for the role of the spine in human locomotion: a challenge to current thinking. Journal of Biomedical Engineering. 1985;7(3):205-216.

[9] Gracovetsky S, Iacono S. Energy transfers in the spinal engine. Journal of Biomedical Engineering. 1987;9(2):99-114.

[10] Gracovetsky S. The spinal engine. Springer, cop. 1989.

[11] Lewis MA, Bekey GA. Gait adaptation in a quadruped robot. Autonomous Robots. 2002;12:301-312.

[12] Leeser KF. Locomtotion experiments on a planar quadruped robot with articulated back spine. [Master's thesis]. Massachusetts Institute of Technology. 1996.

[13] Culha U, Saranli U. Quadrupedal bounding with an actuated spinal joint. In: 2011 ieee international conference on robotics and automation (icra). 2011 May. p. 1392-1397.

[14] Pfeifer R, Bongard J. How the body shapes the way we think: A new view of intelligence. The MIT Press. 2006.

[15] Pfeifer R, Lungarella M, Iida F. Self-organization, embodiment, and biologically inspired robotics. Science. 2007;318(5853):1088-1093.

[16] Zhao Q, Ellenberger B, Sumioka H, Sandy T, Pfeifer R. The effect of spine morphology on a pneumatic-driven quadruped robot for cheetah-like locomotion. In: 2013 ieee international conference on robotics and biomimetics (submitted). 2013.

[17] Zhao Q, Sumioka H, Pfeifer R. The effect of morphology on the spinal engine driven locomotion in a quadruped robot. In: The 5th international symposium on adaptive motion of animals and machines (amam2011). 2011 Oct. p. 51-52.

[18] Zhao Q, Nakajima K, Sumioka H, Yu X, Pfeifer R. Embodiment enables the spinal engine on quadruped robot locomotion. In: 2012 ieee/rsj internattional conference on intelligent robots and systems. 2012 Oct. p. 2449-2456.

[19] Tsujita K, Kobayashi T, Inoura T, Masuda T. Gait transition by tuning muscle tones using pneumatic actuators in quadruped locomotion. In: 2008 ieee/rsj international conference on intelligent robots and systems (iros). 2008 Sept. p. 2453-2458. 
[20] Fischer MS, Witte H. Legs evolved only at the end! Philosophical Transactions of the Royal Society A: Mathematical, Physical and Engineering Sciences. 2007;365(1850):185-198.

[21] Billy. Spinal coord injury resource center. 2012. Available from: http://www.spinalinjury.net/index.html.

[22] Warnecke L. The stem of aplombcpart two: The thoracic spine. 2012. Available from: http://danceadvantage.net/2011/03/09/thoracic-spine/.

[23] Hildebrand M. Motions of the running cheetah and horse. journal of the Royal Society Interface the Royal Society. 1959;40(4):481-495.

[24] Iida F, Pfeifer R. Sensing through body dynamics. Robotics and Autonomous Systems. 2006; 54(8):631-640.

[25] Haupt PL, Haupt SE. Practical genetic algorithms (second edition). John Wiley \& Sons, Inc.2ं004.

[26] Gerritsen KG, van den Bogert AJ, Nigg BM. Direct dynamics simulation of the impact phase in heel-toe running. Journal of biomechanics. 1995;28(6):661-668.

[27] Hackert R, Witte H, Fischer MS. Interactions between motions of the trunk and the angle of attack of the forelimbs in synchronous gaits of the pika (ochotona rufescens). In: Adaptive motion of animals and machines. 2006. p. 69-77.

[28] Brown C, larry Franks. Factors affecting size of young beef cows. Journal of Animal Science. 1964; 23(3):665-668.

[29] Macpherson JM, Ye Y. The cat vertebral column: stance configuration and range of motion. Experimental brain research Experimentelle Hirnforschung Expérimentation cérébrale. 1998 Apr; 119(3):324-32.

[30] Townsend HG, Leach DH, Fretz PB. Kinematics of the equine thoracolumbar spine. Equine veterinary journal. $1983 \mathrm{Apr} ; 15(2): 117-22$.

[31] Schilling N, Hackert R. Sagittal spine movements of small therian mammals during asymmetrical gaits. The Journal of Experimental Biology. 2006;209:3925-3939.

[32] Lee CR, Farley CT. Determinants of the center of mass trajectory in human walking and running. Journal of Experimental Biology. 1998;201(21):2935-44.

[33] Farley CT, Glasheen J, McMahon TA. Running springs: speed and animal size. Journal of Experimental Biology. 1993;185(1):71-86.

[34] He JP, Kram R, McMahon TA. Mechanics of running under simulated low gravity. Journal of Applied Physiology. 1991;71(3):863-870.

[35] Hildebrand M. Symmetrical gaits of horses. Science. 1965;150(3697):701-708.

[36] Raibert M. Symmetry in running. Science. 1986;231(4743):1292-1294.

[37] Alexander RM. Why mammals gallop. American Zoologist. 1988;28(1):237-245.

[38] Caro T. The functions of stotting: a review of the hypotheses. Animal Behaviour. 1986;34(3):649662.

[39] FitzGibbon C, Fanshawe J. Stotting in thomson's gazelles: an honest signal of condition. Behavioral Ecology and Sociobiology. 1988;23(2):69-74.

[40] Caro T. The functions of stotting in thomson's gazelles: some tests of the predictions. Animal Behaviour. 1986:34(3):663 - 684

[41] Pal G, Sherk H. The vertical stability of the cervical spine. Spine. 1988;13(5):447-449. 


\section{Appendix D}

\section{Spine Dynamics as a Computational Resource in Spine-Driven Quadruped Locomotion}

(C)2013 IEEE. Reprinted, with permission, from:

Zhao, Q., Nakajima, K., Sumioka, H., Hauser H., and Pfeifer, R.(2013). Spine Dynamics as a Computational Resource in Spine-Driven Quadruped Locomotion, In the 2013 IEEE/RSJ International Conference on Intelligent Robots and Systems (IROS), pp. 1445-1451.

This is the final accepted version. Final version of the article can be found at http:/ / ieeexplore.ieee.org doi=10.1109/IROS.2013.6696539. 


\title{
Spine dynamics as a computational resource in spine-driven quadruped locomotion
}

\author{
Qian Zhao, Kohei Nakajima, Hidenobu Sumioka, Helmut Hauser, and Rolf Pfeifer
}

\begin{abstract}
Recent results suggest that compliance and nonlinearity in physical bodies of soft robots may not be disadvantageous properties with respect to control, but rather of advantage. In the context of morphological computation one could see such complex structures as potential computational resources. In this study, we implement and exploit this view point in a spine-driven quadruped robot called Kitty by using its flexible spine as a computational resource. The spine is an actuated multi-joint structure consisting of a sequence of soft silicone blocks. Its complex dynamics are captured by a set of force sensors and used to construct a closed-loop to drive the motor commands. We use simple static, linear readout weights to combine the sensor values to generate multiple gait patterns (bounding, trotting, turning behavior). In addition, we demonstrate the robustness of the setup by applying strong external perturbations in form of additional loads. The system is able to fully recover to its nominal gait patterns (which are encoded in the linear readout weights) after the perturbation has vanished.
\end{abstract}

\section{INTRODUCTION}

Traditional robots use rigid materials for structural elements and for actuators, e.g., for their body, arms, and motors. Such rigid body and high torque servos are widely used to allow for precise control and to suppress unwanted dynamics. Although this approach has successfully demonstrated its applicability for achieving various tasks, it requires intensive computation as every degree of freedom has to be precisely controlled at every single time step. Furthermore, these robots perform much worse and less naturally compared to their biological counterparts. In contrast, robots with compliant bodies, could solve this problem, for instance, by applying biologically inspired design to robots to facilitate, e.g., locomotion, while using simple controller [1]-[5]. This indicates that part of the computation need for control can be outsourced to the body by using suitable morphological properties. In this sense, a compliant body may not be a factor to make control hard. Instead, it could be a potential computational resource.

This hypothesis, usually referred to as morphological computation (MC), has recently received some theoretical support by Hauser et al. [6], [7]. They proposed theoretical

This research was funded in party by the European Community's Seventh Framework Programme FP7-ICT-248311 (AMARSi) and FP7-ICT231688 (Locomorph).

Q. Zhao, K. Nakajima, H. Hauser, and R. Pfeifer are with Department of Informatics, University of Zurich, 8050, Zurich, Switzerland, email: \{zhao/nakajima\}@ifi.uzh.ch

K. Nakajima is also with Bio-Inspired Robotics Lab, Swiss Federal Institute of Technology Zurich, 8092 Zurich, Switzerland.

H. Sumioka is with the Advanced Telecommunications Research Institute International. models for MC with compliant bodies, where they demonstrate how compliant physical bodies can be potentially used as a computational resource. They applied the concept of reservoir computing ${ }^{1}$ to random networks of mass-spring systems. Instead of using a neural network or a network of leaky integrators (as in standard approaches for reservoir computing) the previously mentioned models employ a compliant physical body as a reservoir. The theory suggests that complex physical bodies of soft robots could be a potential computational resource, due to their elasticity and nonlinearity inherently embedded in their physical bodies. Additionally, Hauser et al. [7] demonstrated how static feedback from the sensor into the physical body (via actuators) can be used to generate autonomously periodic patterns [5], e.g., as used in locomotion.

In this context, there have been some successful examples of the implementation of the concept of MC. In one case, a simple model of a human musculoskeletal system was used to identify the capacity of computation [9]. In a more biologically plausible example, the computational capacity of a muscular-hydrostat system was investigated and found to have a characteristic memory capacity [10]. In addition, such a system has been demonstrated to have the potential to emulate complex nonlinear dynamical systems, and closed-loop controls [11]. In terms of locomotion, a simulated tensegrity robot has been demonstrated to be capable of embedding nonlinear limit cycles based on different online learning techniques [12]. However, these works are all limited to simulators based on predefined environments and precise and sufficient data collection.

In this study, we implemented this theoretical model to a real spine-driven quadruped robot called Kitty. The impact of real-world conditions on the physical reservoir will be considered, including the partial loss of the state of the morphology, noisy sensory time series, and limited training phase. The spine embedded in Kitty robot is an actuated multi-joint structure consisting of a sequence of compliant silicone blocks and its dynamics is captured by a set of force sensors [13]. Its design is inspired by the biological hypothesis of spinal engine stating that locomotion is mainly achieved by the spine, while the legs may serve as assistance [14], [15].

In this paper, we first introduce a biologically-inspired

${ }^{1}$ Reservoir computing is a machine learning technique used to emulate complex, nonlinear computations by employing a randomly initiated (but afterwards fixed in their parameters) complex, nonlinear dynamical network of nonlinear dynamical systems (i.e., the reservoir). For more details we refer to [8]. 

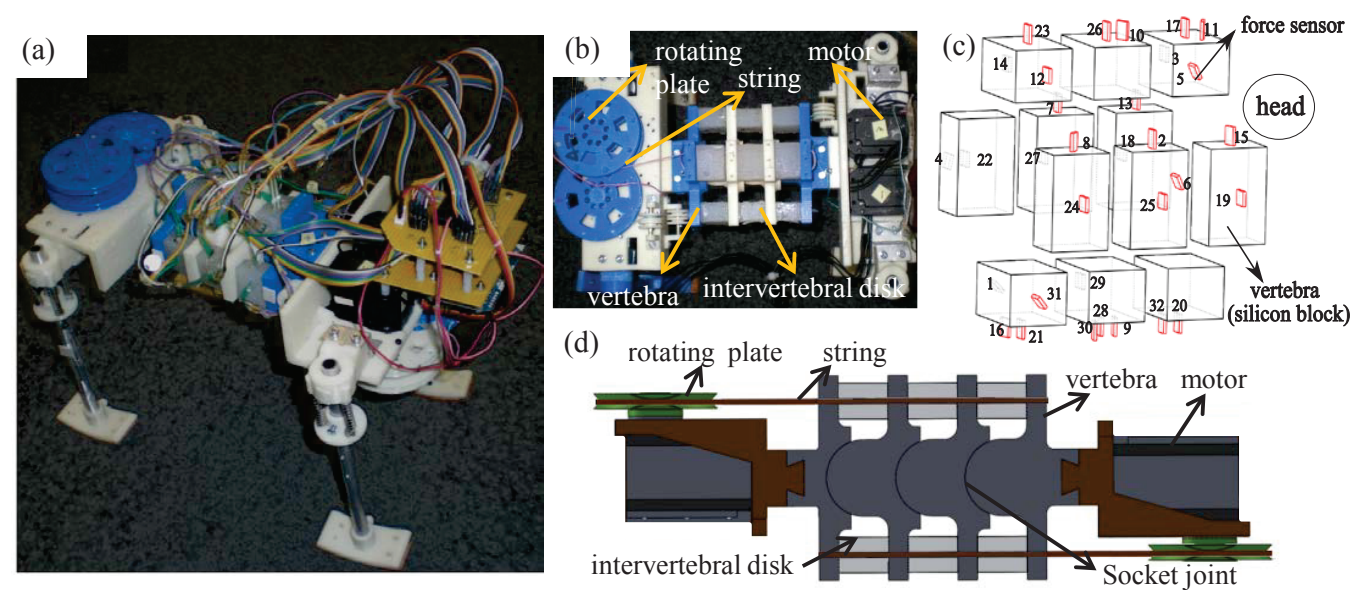

Fig. 1. (a) A quadruped robot equipped with a tendon-driven spine. (b) A biologically inspired spine. (c) The arrangement of 32 force sensors in the spine. Cubic with red contour stands for the force sensor, while cubic with black contour indicates silicone block. (d) Cross section of the artificial spine: sagittal view.

multiple degree-of-freedom spine model [16], [17] to a real quadruped robot and explain its potential to be a computational resource. Second, the experimental procedures are described, including the overall information processing based on MC. Then, experimental results by using actual sensory data from physical robotic system are presented. The results suggest that with the help of the complaint spine (as a computational resource) this MC setup is able to encode movement patterns, produce rhythmic patterns, and learn new pattern. Finally, the robustness of this learned behavior against external perturbation is investigated. We found that noises coming from the real-world conditions benefit the robustness of such a system.

\section{Robot Design}

Kitty is equipped with a flexible spine ( $29 \mathrm{~cm}$ wide, 32 $\mathrm{cm}$ long, $20 \mathrm{~cm}$ high, and $1.4 \mathrm{~kg}$ ) (Fig. 1 (a)). Three springs are mounted in each stick-shaped leg to cushion the shock from the ground. The legs are fixed to the body and have no relative rotation with respect to it. The bottoms of the feet are glued with asymmetrical friction material to guide the walking direction.

Figure 1 (b) shows an artificial spine endowed with biological characteristics. It consists of cross-shaped rigid vertebrae made of ABS plastic, intervertebral disks made of silicone blocks and strings driven by motors, similar to the anatomical spine structure [18]. As shown in Fig. 1 (b), (d), the vertebrae are separated by the silicone blocks, which work as intervertebral discs, and connected by four strings through themselves and the silicone blocks. The four strings connecting vertebrae and intervertebral disks are pulled respectively by four RC motors, which can control the movements of the spine.

Motor command $I_{i}(t)$ to motor $i$ for the spine movements is computed with sinusoid function given by:

$$
I_{i}(t)=A \sin \left(2 \pi f_{i} t+\phi_{i}\right)+\psi \quad i=\{u, d, r, l\},
$$

where $i$ stands for the position of the motor mounted in the robot. $u, d, r$, and $l$ indicate motors controlling the strings located at up, down, right, and left side of the spine, respectively.

The dynamics of the spine is captured by 32 force sensors (FSR400) randomly embedded into silicone blocks (Fig. 1 (c)). The dynamics of this spine is complex due to its flexibility and compliance. In addition, according to the geometrical constraint of the spine configuration, it exhibits highly nonlinear dynamics during locomotion. This suggests the possibility of this compliant spine to be a computational resource and generate diverse locomotive behaviors.

\section{INFORMATION PROCESSING IN FLEXIBLE SPINE}

The task of Kitty is to generate locomotive behaviors by using the dynamics of its spine. The pre-designed motor commands are emulated by static, linear readout units after learning. Figure 2 shows an overview of the information processing based on MC. The robot dynamics are generated by the spine movements driven by four motors. One pattern generator corresponds to one specific locomotive behavior. It consists of four linear readout units (blocks in the area highlighted in grey in Fig. 2) which are associated with motors controlling the up, down, right, left side of the spine. The states of the spine are measured through randomly distributed force sensors (FSR400) in the silicone blocks. Because it is unclear which arrangement of the sensors is the best to perform tasks, we adopted random topology similar to original echo state network.

MC consists of three phases: teaching, learning, and evaluating phases. We take one specific gait $\mathrm{G}$, where $G \in$ 
(a)

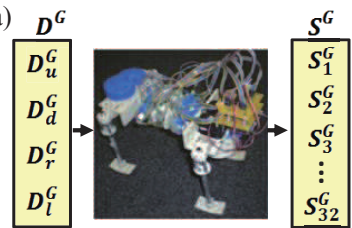

(c)

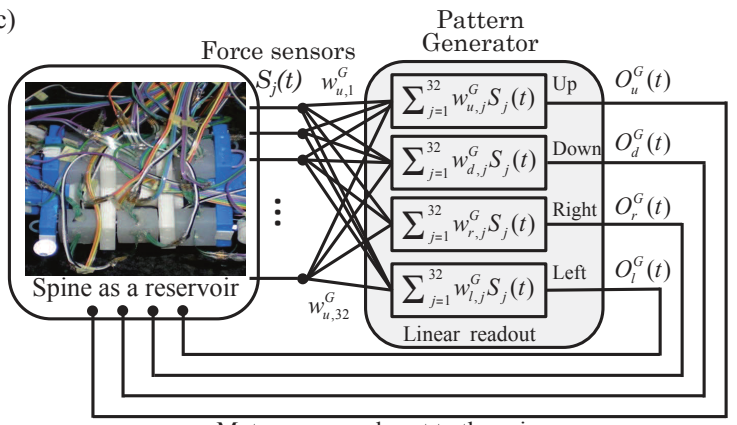

Motor command sent to the spine

Fig. 2. An overview of information processing in the system. (a) Teaching phase (open loop): the predefined motors command $\mathbf{D}^{\mathbf{G}}$ are sent to drive the robot and the associated sensory responses $\mathbf{S}^{\mathbf{G}}$ are collected; (b) learning phase (open loop): linear readouts $\mathbf{w}_{\mathbf{i}}^{\mathbf{G}}$ are adjusted to emulate desired outputs; (c) evaluating phase (close loop): the motor commands $O_{i}^{G}(t)$, where $i \in\{u, d, r, l\}$ are generated by a physical spine and sent to the motors to drive the spine. The motor commands are computed as a sum of current states of force sensors multiplied with output weights $\mathbf{w}_{\mathrm{i}}^{\mathbf{G}}$.

$\{$ bounding,trotting,turningLeft $\}$, as an example to explain the information process.

The teaching phase is implemented in open loop where the motor commands (Eq.1) are sent to the robot and drive it (Fig. 2 (a)). The target signals for four motors in one gait are stored as a vector $\mathbf{D}^{\mathbf{G}}=\left(\mathbf{D}_{\mathbf{u}}^{\mathbf{G}}, \mathbf{D}_{\mathbf{d}}^{\mathbf{G}}, \mathbf{D}_{\mathbf{r}}^{\mathbf{G}}, \mathbf{D}_{\mathbf{l}}^{\mathbf{G}}\right)$, where $u, d$, $r, l$ indicate upside, downside, right side, and left side of the spine, respectively. Accordingly, the associated state $S_{j}(t)$ of force sensor $j$ at every time step $(t=1,2, \cdots, M)$ is collected in a $M \times N$ matrix, $\mathbf{S}^{\mathbf{G}}$, where $N=32$ (the number of force sensors), and $M$ is the time step.

The learning process is carried out with open loop (Fig. 2 (b)). In the learning phase, only the linear readouts are adapted, i.e., $\mathbf{w}_{\mathbf{i}}^{\mathbf{G}}$ are adjusted. The system is forced into the desired motor commands by a "teacher" signal. Therefore, the optimal output weights $\mathbf{w}_{\mathbf{i}}^{\mathbf{G}}$ are calculated by $\mathbf{w}_{\mathbf{i}}^{\mathbf{G}}=$ $\left(\mathbf{S}^{\mathbf{G}}\right)^{+} \mathbf{D}^{\mathbf{G}}$, where $\left(\mathbf{S}^{\mathbf{G}}\right)^{+}$stands for the (Moore-Penrose) pseudo-inverse of $\mathbf{S}^{\mathbf{G}}$.

In the evaluating phase, the loops are closed (Fig. 2 (c)). Spine dynamics are sent to linear and static readout units that compute outputs of the system $O_{i}^{G}(t)$, where $i \in\{u, d, r, l\}$. For each linear readout, $O_{i}^{G}(t)$ is a sum of values of the force sensors $S_{j}(t)$ multiplied by output weights $\mathbf{w}_{\mathbf{i}}^{\mathbf{G}}=\left(w_{i, 1}^{G}, w_{i, 2}^{G}, \cdots, w_{i, 32}^{G}\right): O_{i}^{G}(t)=\sum_{j=1} w_{i, j}^{G} S_{j}(t)$. In this formula, $w_{i, j}^{G}$ indicate the output weight for $\mathrm{j}$-th force sensor for linear output $\mathrm{i}$, and $S_{j}(t)$ is the value of the $\mathrm{j}$-th sensor at time $t$.

\section{EXPERIMENTAL SETTING}

\section{A. Teaching signals}

The target signals in this physical reservoir computing are the commands sent to the motors located at the up, down, right, and left side of the spine, which control spine movements. The spine of Kitty robot is controlled with periodic motor commands given by Eq.1 using parameters shown in Table I. Note that motors whose parameters are marked with asterisks in the table are controlled with constant values to keep the natural length without stretching and relaxing.

TABLE I

CONTROLLERS FOR SPINE MOVEMENTS

\begin{tabular}{|c|c|c|c|c|}
\hline controller & $\left(f_{u}, \phi_{u}\right)$ & $\left(f_{d}, \phi_{d}\right)$ & $\left(f_{r}, \phi_{r}\right)$ & $\left(f_{l}, \phi_{l}\right)$ \\
\hline$S_{\text {bounding }}$ & $\left(\frac{1}{\pi}, 0.0\right)$ & $\left(\frac{1}{\pi}, \pi\right)$ & $*$ & $*$ \\
\hline$S_{\text {trotting }}$ & $*$ & $*$ & $\left(\frac{1}{\pi}, \pi\right)$ & $\left(\frac{1}{\pi}, 0.0\right)$ \\
\hline$S_{\text {turningLeft }}$ & $\left(\frac{1}{\pi}, 0.0\right)$ & $\left(\frac{1}{\pi}, \pi\right)$ & $\left(\frac{1}{\pi}, 0.0\right)$ & $\left(\frac{1}{\pi}, \pi\right)$ \\
\hline
\end{tabular}

The bounding gait, as a result of spine flexion-extension movements, is generated by the controller $S_{\text {bounding }}$ [13]. This controller only pulls the strings located at the upside and downside of the spine alternately, while the side strings are kept the natural length. Similarly, the trotting gait, generated by spine lateral movements, can be achieved by controller

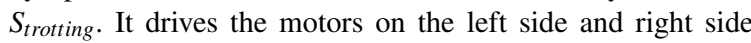
alternately. Turning left behavior, controlled by $S_{\text {turningLeft }}$, can be realized by combining bounding gait and trotting gait together. The only difference between turning right and left is the flip between $\phi_{r}$ and $\phi_{l}$, which are the phase lags with respect to the upside of the spine.

\section{B. Experimental procedures}

To achieve MC, three phases are used: teaching, learning, and evaluating phases. In the teaching phase, the number of teaching data used to train the reservoir readouts is 600 time steps after initial 200 samples are discarded as transients. The number of sampling data for one cycle is heuristically set to 20 .

\section{RESUlts}

In this section, the behavior of the physical reservoir is observed first. Then three different resultant locomotive patterns are analyzed. In the end, robustness of this reservoir is studied by adding external load on the robot.

\section{A. Versatile behaviors using the same physical body}

Figure 3, 4 show the sequential pictures of bounding gait, turning left behavior in the evaluating phase, as a result of pronounced spine movements. The signals generated by such a spine reservoir are able to drive the robot to emulate a specific gait.

Figure 5, 6, and 7 show the best performance of the spine reservoir, associated sensory response, and footfall pattern in bounding gait, trotting gait, and turning left behavior, respectively. We found that the generated control signals are periodic and similar to the desired ones in terms of the frequency and the shape (Fig. 5 (a), 6 (a), and 7 (a)). 


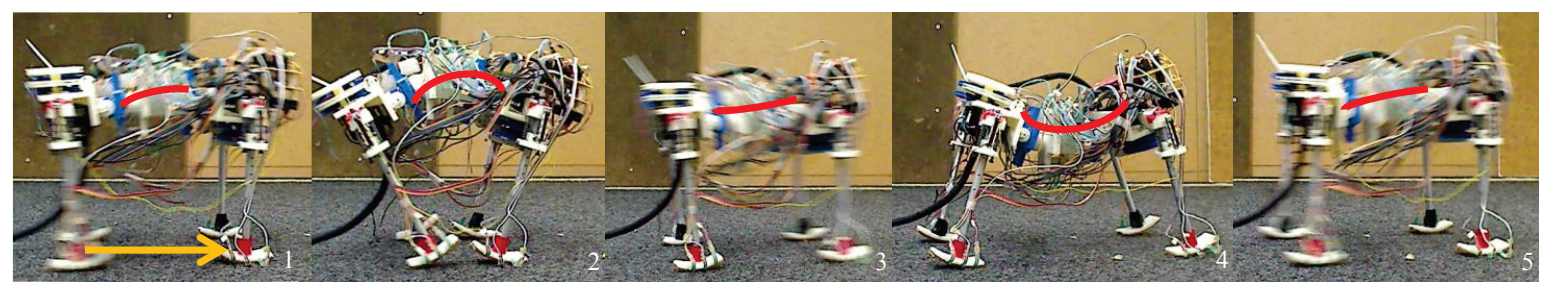

Fig. 3. Sequential pictures of spine movements in the bounding gait in the evaluating phase. Orange arrow represents the walking direction.

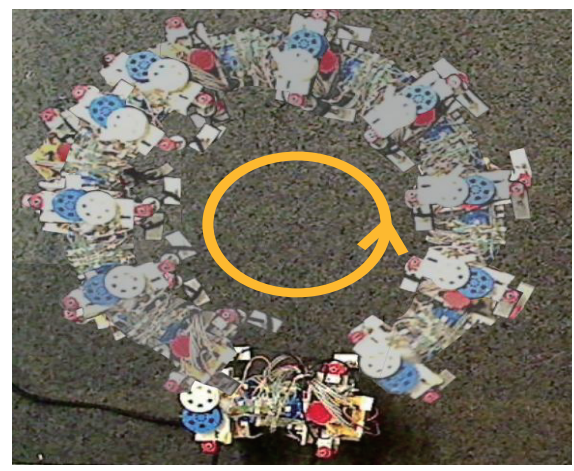

Fig. 4. Sequential pictures of spine movements in turning left behaviour in the evaluating phase. Orange arrow represents the walking direction.

Figure 5 (b), 6 (b), and 7 (b) suggest that the dynamics of this spine has a specific correspondence to each behavior, i.e., dynamic of each side of the spine (sensory values of all sensors located in each side) has different patten according to each behavior. For instance, the sensors located at the upside and downside response more than the ones at the right and left side in the bounding gait, as a result of sagittal spine movements (Fig. 5 (b)). Lateral spine movements result in trotting gait. As a consequence, sensory responses at right and left side of the spine are much higher than the responses captured in the up and down side (Fig. 6 (b)). Since turning left behavior emerges when bounding and trotting gait are combined together, the sensory responses are also a combination from bounding and trotting gait (Fig. 7 (b)).

We also noticed that the actual signals cannot very precisely emulate the desired signals. This is due to the limitation of physical platform and arena. For example, the motors easily get hot and stop working after 2,000 teaching time steps, or the robot is sensitive to the terrain because of the lack of ground clearance. However footfall patterns clearly show that the legs are coupled correctly to achieve bounding, trotting, and turning left behaviors, even if little phase delay and error exist (Fig. 5 (c), 6 (c), and 7 (c)). ${ }^{2}$ In

${ }^{2}$ In this paper, we did not adopt measures evaluating the difference between the target and actual commands, such as Mean Square Error (MSE). This is because we often observed a case that the actual motor command generate a seemingly correct motor command with phase shift, compare with the target motor command. When we use MSE for example, this effect avoids the appropriate evaluation of the actual motor commands.

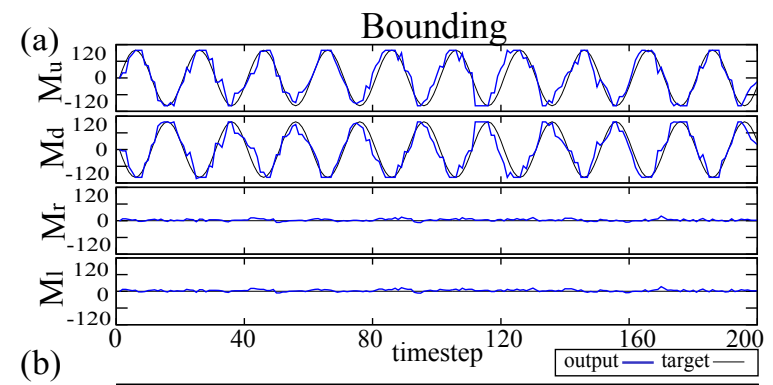

(b)

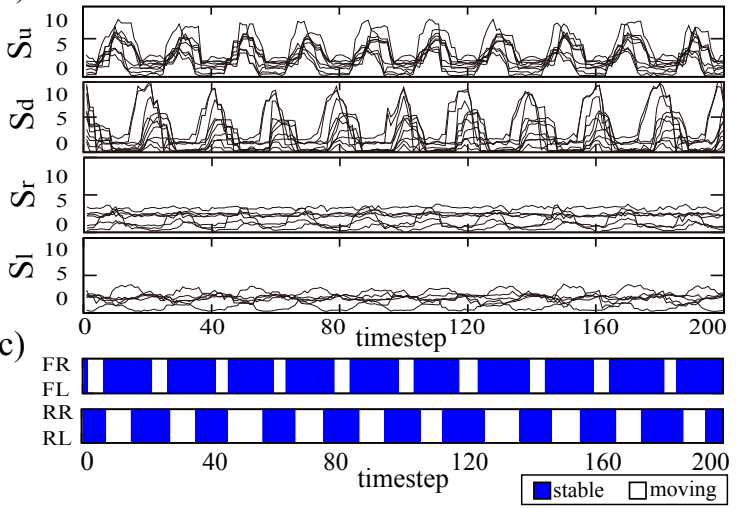

Fig. 5. (a) The performance of pattern generator in evaluating phase: bounding gait. Four subplots from top to bottom are the results of pattern generators for motor controlling the up, down, right, left side of the spine, respectively. Y-axis stands for the amplitude sent to the motor. X-axis indicates the time steps. The grey thin curve is the target trajectory and the blue thick curve is the actual output from the spine reservoir. (b) Sensory responses in evaluating phase. Four subplots from top to bottom are sensory responses collected at the up, down, right, left side of the spine, respectively. $\mathrm{Y}$-axis stands for the force $[\mathrm{N}]$ measured from the sensors. (c) Footfall patterns in evaluating phase: bounding gait (FR: front right leg; FL: front left leg; RR: rear right leg; RL: rear left leg).

this paper, we define the footfall pattern based on whether the feet move forward or keep stable, because Kitty robot does not have any actuation on the legs, especially knee joints which mostly contribute to lift up the feet and produce ground clearance.

Figure 8 shows the obtained readout weights for each gait. The weights are adjusted in the learning phase and then are fixed in the evaluating phase. We observed that in bounding gait (Fig. 8 (a)), the weights associated with the up-down motors have higher values than the ones with right- 


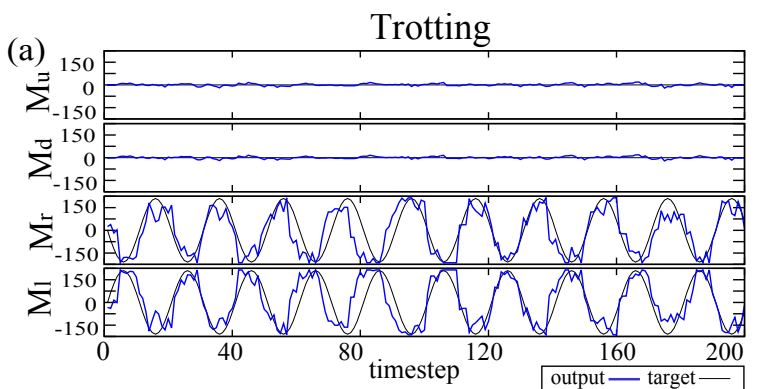

(b)

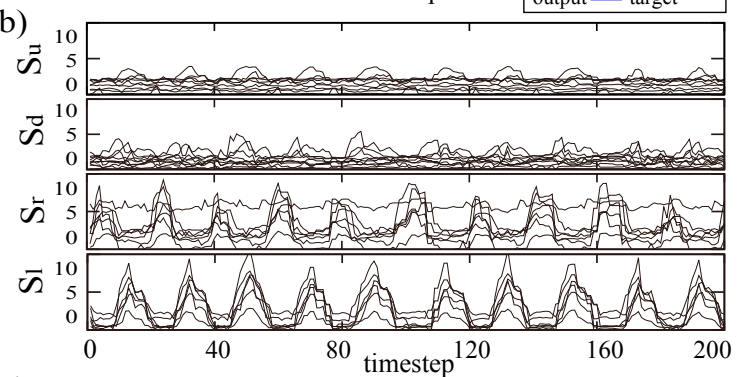

(c) ${ }_{\mathrm{RL}}^{\mathrm{FR}}$

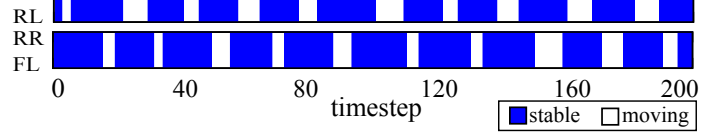

Fig. 6. (a) The performance of pattern generator in evaluating phase: trotting gait. $\mathrm{X}$ and $\mathrm{Y}$ axes in four subplots represent the same meaning as Fig. 5 (b) Sensory responses in evaluating phase. Y-axis stands for the force $[\mathrm{N}]$ generated by the sensors.(c) Footfall patterns in evaluating phase: trotting gait (FR: front right leg; FL: front left leg; RR: rear right leg; RL: rear left leg).

left motors. The motors controlling strings located at right and left sides do not contribute to the bounding gait so much. This is also reflected in the readout weights for right and left motors, whose values are close to zero and overlap with each other. The weights for up motors are nearly mirror image of the weights for down motors about dashed line. The results reveal that the weights can reflect the coordination among the motors. Similarly, in trotting gait, the weights of linear readouts for up and down motors are nearly zero, while the weights of linear readout for right and left motors are almost symmetrical about dashed line. In turning left behavior, the weights of linear readouts for right and down motors are overlapped, and the same for the rest two sets.

These results suggest that this compliant spine can be regarded as a computational device to generate repetitive movements, in addition to be a mechanical component connecting the front legs and rear legs. Indeed, multiple behaviors can be produced by the same physical body, only by adjusting the linear readouts.

B. Robustness against external perturbation in bounding gait

One crucial criterion in evaluating the performance of learning is robustness against external perturbation. There-
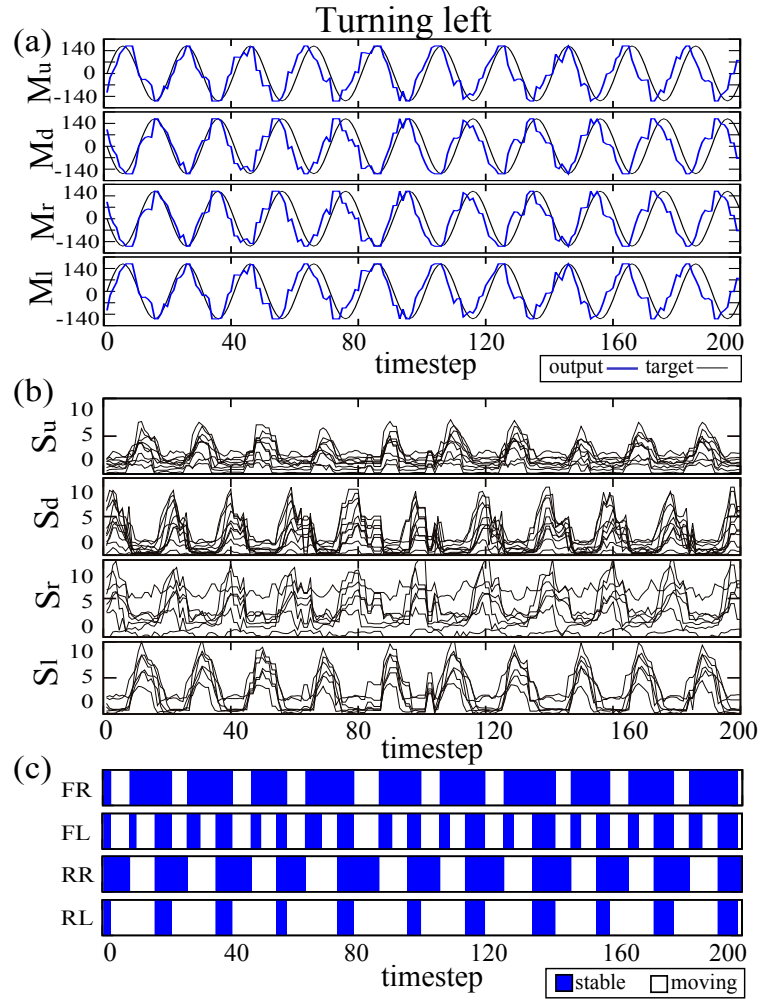

Fig. 7. The performance of pattern generator in evaluating phase: turning left behavior. $\mathrm{X}$ and $\mathrm{Y}$ axes in four subplots represent the same meaning as Fig. 5 (b) Sensory responses in evaluating phase. Y-axis stands for the force $[\mathrm{N}]$ generated by the sensors. (c) Footfall patterns in evaluating phase: turning left behavior (FR: front right leg; FL: front left leg; RR: rear right leg; RL: rear left leg).
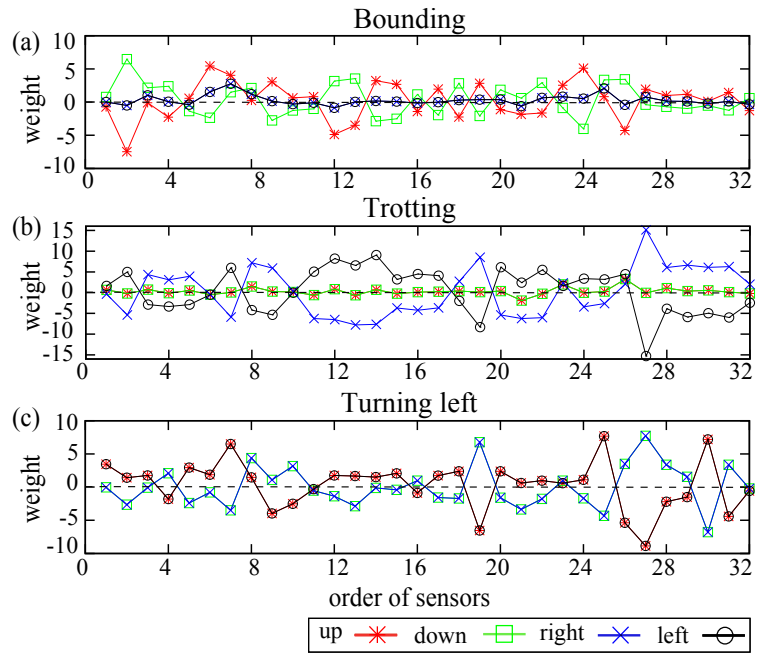

Fig. 8. The weights of linear readout trained in the learning phase in bounding gait (a), trotting gait (b), and turning left behavior (c). $\mathrm{X}$ axis stands for the order of the sensors, and y axis represents the value of weights. 
fore, it is important to test how robust the spine reservoir is against external perturbation. We tested this characteristic by adding load in the front part of the body (Fig. 9 (a)). The number of evaluating phase is 360 time steps. In the first 40 time steps, the robot is moving without any load. At time step 40, an external load is added to the front body and remains until time step 200. Different loads ranging from $100[\mathrm{~g}]$ to $1000[\mathrm{~g}]$ have been tested in this spine reservoir, as shown in Fig. 9 (b). The experiments were conducted five trials. The average speed and the standard derivation were recorded when the external load is applied on the robot for 160 time steps. The results suggest that the speed of Kitty has a negative correlation with the external load. We observe that with the increase of the load, the performance of the spine reservoir gets affected more. In other words, the generated signals more easily get stuck at some points. Accordingly, the robot vibrates at these postures. This vibrating movements do not contribute to the speed too much and might account for the resultant slower speed. In addition, the stability gets worse with the increase of external loads.

Figure 9 (c) shows two typical cases: one is with load $400[\mathrm{~g}]$ and the other is with $1000[\mathrm{~g}]$. In the former case, when the external load is added, the amplitude of the signals generated by the spine reservoir gets suppressed in the following three cycles, but the frequency still remains the same. From time step 100, the spine reservoir recovers its repetitive performance. After the load is moved, the performance of this physical reservoir get affected again and the amplitude drops. It starts to recover after two cycles. However in the latter case, the load is too heavy, almost two-thirds of Kitty robot's own weight. Thus, this load stops reservoir's performance. But once it is removed, the ability of emulating desired signal is restored and locomotive pattern continues. This good performance of the learned behavior against external perturbation might be accounted for the noise, which is inherent to Kitty robot.

Because robustness can be enhanced by manually added noise in teaching phase in the simulator [7], we think that the observed good performance against external perturbation might be accounted for noise, which is inherent to Kitty robot.

\section{Discussion AND Conclusion}

In this paper, we demonstrated that the developed compliant spine structure is not only a mechanical component connecting the front legs and rear legs, but rather can also serve as a computational resource to achieve different behaviors, such as bounding gait, trotting gait, and turning behavior. The results suggest that this computational resource (compliant spine) together with linear and static readouts and feedback loops is able to encode movement patterns, produce rhythmic patterns, and learn new pattern. Remarkably, multiple behaviors can be produced by the same fixed physical body, simply by readjusting the weights of the linear readouts. In addition, we demonstrated the robustness of the learned behavior by applying additional load as external perturbations. The results show that this system is able to (a)
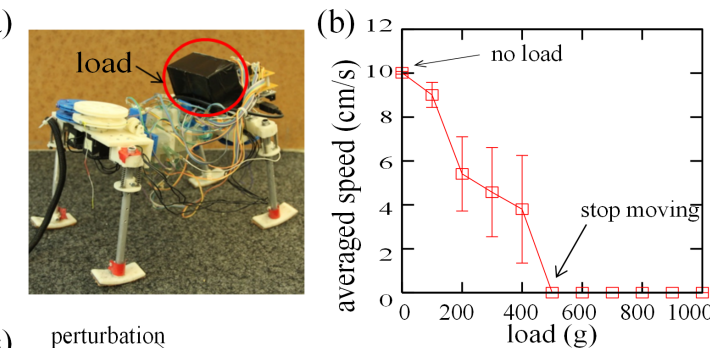

(c) perturbation load $(\mathrm{g})$

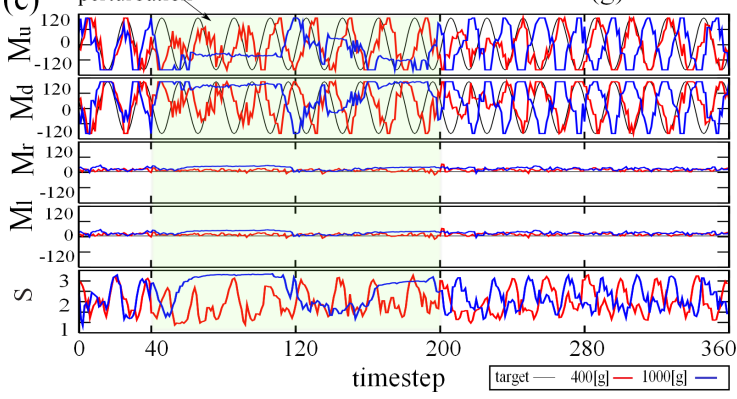

Fig. 9. (a) Kitty robot with external load. (b) Stability of spine reservoir against various external loads in bounding gait. (c) The performance of spine reservoir in evaluating phase with external load in bounding gait. The top four subplots are the comparison between target signal and actual output signal from reservoir for the motor controlling the up, down, right, left side of the spine, respectively. The bottom subplot is the dynamics of the spine which is average value over 32 force sensors. Red curve represents the case when external load weighing $400[\mathrm{~g}]$ is applied, while blue curve is the case with the load of 1000 [g]. Grey curve is the target signal. X-axis indicates the time steps. The area marked in green is the period when the spine reservoir is disturbed. In the top four subplots, Y-axis stands for the amplitude [degree] sent to the motor. In the bottom subplot, Y-axis stands for the force $[\mathrm{N}]$.

recover to its nominal gait patterns encoded in the linear weights after the perturbation has disappeared.

In our experiments, although we were able to observe a successful locomotion for each gait pattern, the produced motor commands were noisy and were unable to emulate the target commands precisely. This is mainly caused by the limitation of this platform when it starts to interact with the environment. For instance, the amount and quality of the teaching data available in physical platform is much lower than in comparable simulated work. In spite of it, the spine reservoir was still able to produce stable and seemingly periodic locomotive patterns.

A possible solution to reduce the error between the target and the output signals is to optimize the spine structure by employing more asymmetrical features. This is inspired by biology, where asymmetrical spine structure can be observed in animals. Such features are unevenly distributed muscles in the spine, asymmetrical muscle stiffness, and the shape of the spinal column [19]. These properties could increase the diversity and nonlinearity of the spine reservoir and improve its performance. In addition, in order to better reflect the spine dynamics in the sensory time series, the number and the locations of the sensors within the spine can be explored and optimized in future work. 
Another future research direction is to ask whether a gait switching could be achieved in our framework [20], [21]. One possible scenario would be to explore ways to embed multiple gaits with a single fixed linear readout and a feedback loop by introducing an input signal corresponding to each gait to the spine. The signal acts as an initiation signal (or a control signal) for the gait switching and would be provided either as an external or internal control command [7]. Especially in our contexts, the signals can be mechanical, such as an intentional movements of a head or a tail of the robot's body, or can be also generated from an environmental change, such as the change of the terrain.

\section{REFERENCES}

[1] F. Iida and R. Pfeifer, "Sensing through body dynamics," Robotics and Autonomous Systems, vol. 54, no. 8, pp. 631-640, 2006

[2] S. Kim, J. E. Clark, and M. R. Cutkosky, "isprawl: Design and tuning for high-speed autonomous open-loop running," The International Journal of Robotics Research, vol. 25, no. 9, pp. 903-912, 2006.

[3] K. Hosoda, T. Takuma, A. Nakamoto, and S. Hayashi, "Biped robot design powered by antagonistic pneumatic actuators for multi-modal locomotion," Robotics and Autonomous Systems, vol. 56, no. 1, pp. 46-53, 2008.

[4] R. Pfeifer and J. Bongard, How the Body Shapes the Way We Think: A New View of Intelligence. The MIT Press, 2006.

[5] R. Pfeifer, M. Lungarella, and F. Iida, "Self-organization, embodiment, and biologically inspired robotics," Science, vol. 318, no. 5853, pp. 1088-1093, 2007

[6] H. Hauser, A. Ijspeert, R. Fuechslin, R. Pfeifer, and W. Maass, "Towards a theoretical foundation for morphological computation with compliant bodies," Biological Cybernetics, vol. 105, pp. 355-370, 2011.

[7] — "The role of feedback in morphological computation with compliant bodies," Biological Cybernetics, vol. 106, pp. 595-613, 2012.

[8] B. Schrauwen, D. Verstraeten, and J. Van Campenhout, "An overview of reservoir computing: theory, applications and implementations," in Proceedings of the 15th European Symposium on Artificial Neural Networks, 2007, pp. 471-482.

[9] H. Sumioka, H. Hauser, and R. Pfeifer, "Computation with mechanically coupled springs for compliant robots," in Intelligent Robots and Systems (IROS), 2011 IEEE/RSJ International Conference on, Sept., pp. $4168-4173$.

[10] K. Nakajima, H. Hauser, R. Kang, E. Guglielmino, D. G. Caldwell, and R. Pfeifer, "Computing with a muscular-hydrostat system," in 2013 IEEE International Conference on Robotics and Automation (ICRA), May 2013, pp. 1496-1503

[11] — - "A soft body as a reservoir: case studies in a dynamic model of octopus-inspired soft robotic arm," Frontiers in Computational Neuroscience, vol. 7, no. 91, pp. 1-19, 2013.

[12] K. Caluwaerts, M. D'Haene, D. Verstraeten, and B. Schrauwen, "Locomotion without a brain: Physical reservoir computing in tensegrity structures," Artificial Life, vol. 19, no. 1, pp. 35-66, 2013.

[13] Q. Zhao, K. Nakajima, H. Sumioka, X. Yu, and R. Pfeifer, "Embodiment enables the spinal engine on quadruped robot locomotion," in 2012 IEEE/RSJ Internattional Conference on Intelligent Robots and Systems, Oct. 2012, pp. 2449-2456.

[14] S. Gracovetsky and S. Iacono, "Energy transfers in the spinal engine," Journal of Biomedical Engineering, vol. 9, no. 2, pp. 99-114, 1987.

[15] S. Gracovetsky, The Spinal Engine. Springer, cop, 1989.

[16] Q. Zhao, H. Sumioka, and R. Pfeifer, "The effect of morphology on the spinal engine driven locomotion in a quadruped robot," in The 5th International Symposium on Adaptive Motion of Animals and Machines (AMAM2011), Oct. 2011, pp. 51-52.

[17] Q. Zhao and H. Sumioka, "The effect of robot morphology on locomotion from the perspective of spinal engine in a quadruped robot," in International Conference on Morphological Computation, Sep. 2011, pp. 130-132.

[18] R. Pashman and T. Kim, "Normal spinal anatomy," 2012. [Online]. Available: http://www.espine.com/anatomy-normal.htm
[19] R. M. Alexander and A. S. Jayes, "Estimates of the bending moments exerted by the lumbar and abdominal muscles of some mammals," Journal of Zoology, vol. 194, no. 3, pp. 291-304, 1981.

[20] D. Owaki, L. Morikawa, and A. Ishiguro, "Listen to body's message: Quadruped robot that fully exploits physical interaction between legs," in Intelligent Robots and Systems (IROS), 2012 IEEE/RSJ International Conference on, 2012, pp. 1950-1955.

[21] A. J. Ijspeert, A. Crespi, D. Ryczko, and J.-M. Cabelguen, "From swimming to walking with a salamander robot driven by a spinal cord model," Science, vol. 315, no. 5817, pp. 1416-1420, 2007. 


\section{Appendix E}

\section{The Effect of Spine Actuation and Stiffness on a Pneumatically-driven Quadruped Robot for Cheetah-like Locomotion}

(C)2013 IEEE. Reprinted, with permission, from:

Zhao, Q., Ellenberger, B., Sumioka, H., Sandy, T., and Pfeifer, R.(2013). The Effect of Spine Actuation and Stiffness on a Pneumatically-driven Quadruped Robot for Cheetah-like Locomotion, In the 2013 IEEE International Conference on Robotics and Biomimetics (ROBIO), pp. 1807-1812.

This is the final accepted version. Final version of the article can be found at http:/ / ieeexplore.ieee.org (doi=10.1109/ROBIO.2013.6739730). 


\title{
The Effect of Spine Actuation and Stiffness on a Pneumatically-driven Quadruped Robot for Cheetah-like Locomotion
}

\author{
Qian Zhao, Benjamin Ellenberger, Hidenobu Sumioka, Timothy Sandy, and Rolf Pfeifer
}

\begin{abstract}
Biological research has concluded that the actuation of the spine contributes significantly to the performance of quadrupeds in terms of controlling body posture, and integrating limbs and trunk actions. Inspired by this biological findings, we develop a pneumatically-driven quadruped robot called Renny with configurable spine morphology to study how the spine contributes to cheetah-like running. Three spine morphologies: rigid spine, passive spine, and actuated spine, are introduced and tested in Renny robot. In addition, we investigate the effect of the stiffness distribution of the spine muscles in the passive case. The experimental results show that the passive one where the dorsal stiffness is higher than the ventral stiffness can run faster, even faster than the rigid case. Moreover, the coordination between the legs and the actuated spine is studied in actuated spine morphology. We found that when the spinal movements are synchronized with the legs movements, the speed is much faster. In the actuated case, both flexion and extension benefit the increase of the speed by advancing limbs rapidly and increasing the limb swing.
\end{abstract}

\section{INTRODUCTION}

Legged robotics has drawn much more attention from robotic researchers due to its applications in rough terrains in nature and in our living environments [1]. Most of the existing quadruped robots are very similar in their morphology, and feature a single rigid body with four legs with individually actuated hips and/or knees. However, the resulting locomotion behavior is much more constrained than its natural counterpart in terms of speed, energy efficiency, maneuverability, and adaptivity to rough terrain.

From a biological point of view, one of the major differences between robots and quadruped animals is the spine. It is central to control the body posture, provide the foundation to produce the legs' movement, and integrate limbs and trunk actions [2]. For example, a cheetah, the fastest animal in the land, is able to reach up to $110[\mathrm{~km} / \mathrm{h}]$ for a short dash. This is contributed by the marked flexion and extension of the spine to a large extent, which increases the swing of the limbs, advances the limbs more rapidly, thus leading to greater power and speed [3].

Inspired by such biological findings, recently, there have been a few attempts to introduce a spinal joint to a quadruped robot, with the aim of improving the performance of existing robots [4]-[6]. However, most researchers only focused on

This research was funded by the European Community's Seventh Framework Programme FP7-ICT-248311(AMARSi).

Q. Zhao, B. Ellenberger, and R. Pfeifer are with Department of Informatics, University of Zurich, 8050, Zurich, Switzerland, email: zhaodifi.uzh.ch

T. Sandy is with Swiss Federal Institute of Technology Zurich.

H. Sumioka has moved to Advanced telecommunications research institute international. the design of the controller of this spinal joint, and barely paid attention to its morphology. As a result, this spinal joint is usually designed to be electric motor-driven and has less possibility to be morphologically explored. However, in fact, from the embodiment point of view, intelligence requires a body. This suggests that robots designed by clever morphology and proper use of materials system are usually simpler, more robust and adaptive than those based on the classical control paradigm [7]. Furthermore, Boszczyk has pointed out the importance of spine morphology and suggested that a mammalian lumbar spine is an exact accommodation to specific biomechanical demands i.e., locomotion mode depends on spine morphology in nature [8].

In this vein, the effect of the stiffness of the spinal joint in quadruped gait transition has been studied and the results show that suitable spine stiffness makes smooth and stable autonomous gait transition possible [9] [10]. A spine structure with variable viscoelasticity embedded to a quadruped robot might be a suitable body design facilitating multi-modal locomotion at low computational costs [11]. Despite these previously done work, there is still plenty of room for further exploration on the spine morphology. If we look back to the anatomical structure of a biological spine, we can find that there still exist other more critical spine morphological features worthy investigating further, except the ones discussed above, to gain deep knowledge of the working mechanism of the spine.

In this paper, a pneumatically-driven quadruped robot named Renny is developed to mimic cheetah-like running. To achieve this goal, a spine with configurable morphology is designed and mounted to this robot to investigate how the spine morphology contributes cheetah-like running. This spine is designed such that it can easily change to be rigid, passive, or actuated case. In addition, more morphologies, e.g., actuation and stiffness distribution of the spine muscles, and the coordination between the spine and legs are studied to understand how they affect locomotion.

\section{ROBOT DESIGN}

An overview of the whole robot, the basic features of pneumatic artificial muscle, leg and spine morphology, electro-pneumatic system, and controller design are presented in this section.

\section{A. Overview}

Figure 1 depicts the mechanical design of this quadruped robot named Renny. It has nine degrees of freedom (DoFs) which are four hip joints, four knee joints, and one spinal 
(a)

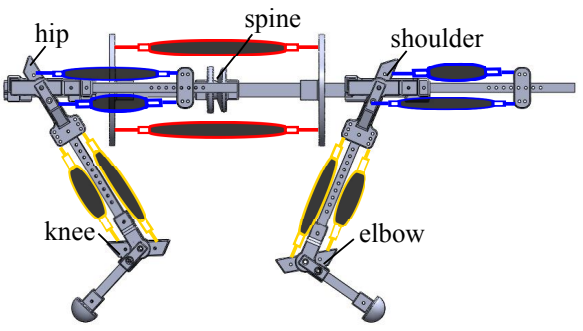

(b)

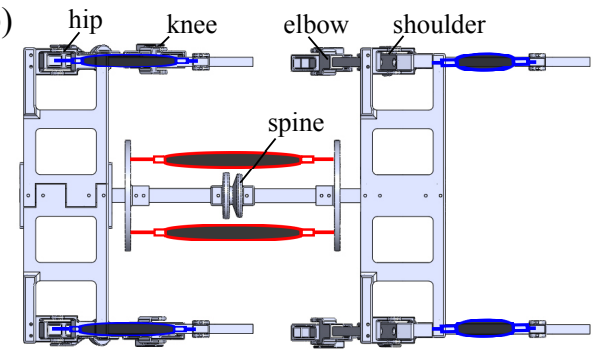

Fig. 1. Schematic of Renny: side view (a) and top view (b). PAMs actuating knee joint, hip joint, and spinal joint are represented by orange, blue, and red contour, respectively.

joint. Each DoF is actuated by a pair of antagonistic pneumatic artificial muscles (PAMs). Renny was designed such that its body morphology can be adjusted easily to be either stiff, passive with tunable stiffness, or actuated. This configurability provides the ability to investigate the effect of body morphology on locomotive performance. Basic specifications of Renny are presented in Table I.

\begin{tabular}{|r||l|}
\hline \multicolumn{2}{|c|}{ Morphology } \\
\hline Size & $60 \mathrm{~cm} \mathrm{x} \mathrm{30} \mathrm{cm} \mathrm{x} \mathrm{35} \mathrm{cm}$ \\
Weight of the robot & $1.85 \mathrm{~kg}$ \\
Antagonistic muscles & 12 pairs \\
Material & ABS Plastic, Aluminium profile \\
\hline \multicolumn{2}{|c|}{ Length } \\
\hline shoulder - elbow & $25 \mathrm{~cm}$ \\
elbow - forefoot & $14 \mathrm{~cm}$ \\
hip - knee & $25 \mathrm{~cm}$ \\
knee - hindfoot & $14 \mathrm{~cm}$ \\
hip muscle & $18 \mathrm{~cm}$ \\
knee muscle & $17 \mathrm{~cm}$ \\
spine muscle & $18 \mathrm{~cm}$ \\
\hline \multicolumn{2}{|c|}{ Controller } \\
\hline CPU board & Arduino Mega \\
Valves & FESTO MPYE-5-1/8-HF-010-B \\
\hline & TABLE I
\end{tabular}

SPECIFICATION OF RENNY

\section{B. Pneumatic Artificial Muscles (McKibben Type)}

PAMs have been widely used in robotics, due to their inherent compliance, high power-weight ratio, muscle-like
TABLE II

SPECIFICATION OF THE MUSCLE

\begin{tabular}{|l|l|l|}
\hline Parameter & Hip\&Spine & Knee \\
\hline Diameter of rubber inner layer & $5.6 \mathrm{~mm}$ & $5.6 \mathrm{~mm}$ \\
Length of rubber inner layer & $60 \mathrm{~mm}$ & $50 \mathrm{~mm}$ \\
Diameter of braided mesh sleeve & $21 \mathrm{~mm}$ & $21 \mathrm{~mm}$ \\
Length of braided mesh sleeve & $125 \mathrm{~mm}$ & $95 \mathrm{~mm}$ \\
Interweave angle of braided mesh sleeve & $133^{\circ}$ & $130^{\circ}$ \\
\hline
\end{tabular}

Note: Length of braided mesh sleeve is defined as the natural length between the two metal wires used to bond PAMs.

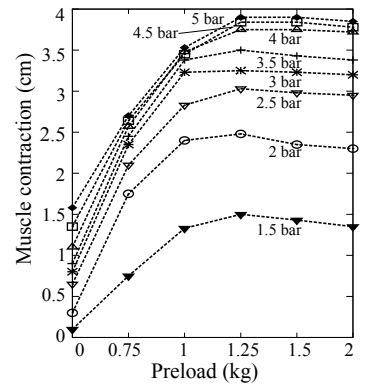

(a)

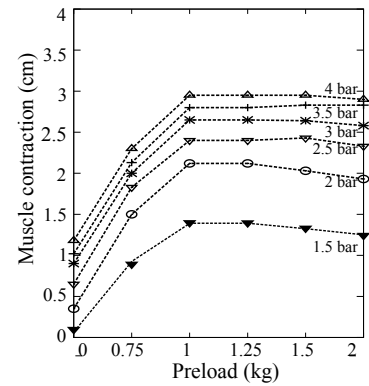

(b)
Fig. 2. Specification of pneumatic artificial muscles for hip and spinal joint (a), and for knee joint (b).

force-length properties [12] [13]. One of the well-known PAMs is McKibben artificial muscle which consists of a rubber tube that is surrounded by a tubular braided fiber mesh sleeve. When the rubber tube is pressurized and inflated, the mesh expands radially and contracts axially, shortening the overall length of the muscle and subsequently producing a pulling force. In the following robot design, we use McKibben artificial muscles specified in Table II to actuate spine, hip, and knee joints.

Experiments were first conducted to understand the basic features of McKibben muscles, e.g., how varying the air supply pressure and preload affects muscle deformation. Since it is very complex to calculate the stiffness of PAMs in dynamic case [14], we only focus on the analysis on the static properties of PAMs. Because stiffness can be adapted by pressure and they are positively correlated [13] [14], it is reasonable to use pressure to replace stiffness.

In the experiments, the air supply end of the muscle was rigidly attached to a table, and a mass was hung from the free end. In each test, the contraction over sequential cycles was then averaged. First, we observe that contraction increases significantly as supply pressure increases (Fig. 2). Secondly, the results show that there exists the same tendency between contraction and preload for each pressure. The maximal contraction increases as the preload is increased up to a threshold where the actuator is nearly fully stretched (1.25 $[\mathrm{kg}]$ for hip and spine muscle, and 1 [kg] for knee muscle). Beyond this threshold, the contraction decreases. Last, hip and spine muscles with higher ratio of the outer sleeve to the inner tube exhibits the higher contraction capability, compared to knee muscles. 

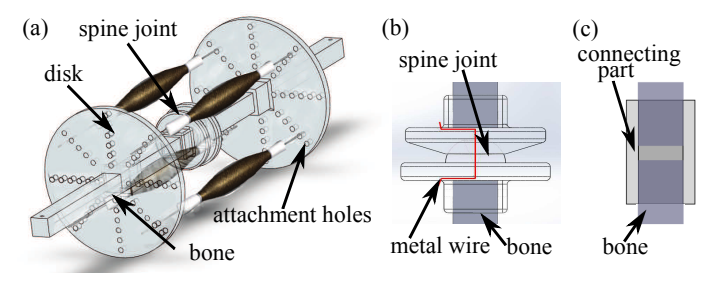

Fig. 3. (a) Musculoskeletal structure of the spine. (b) Spinal joint with the channel for the connecting wire highlighted in red. (c) Connecting part for rigid spine.

\section{Leg Design}

The legs were designed such that their morphologies can be easily adjusted without the need for additional hardware. Multiple attachment holes (Fig. 1(a)), which are provided for each actuator on the skeletons, allow the antagonistic actuators to have different mounted lengths. This gives the ability to offset the position of the leg, and limit the range of motion of the joint in one desired direction.

\section{Spine Design}

To investigate the effect of actuation and stiffness distribution of the muscles in the spine, Renny's spine was designed for full reconfigurability. For example, the length of the spine, and the location to mount the spine muscles can be easily tuned. Furthermore, it can also be changed to a rigid, passive, or actuated spine without difficulty.

In the spine design, one ball and socket joint was used to simplify joint motion (Fig. 3(a),(b)), to make it easier to analyze the body dynamics during experiments. Two circular ABS discs mounted on either side of the spinal joint allow up to eight pneumatic actuators to be mounted in various configurations around the spine (Fig. 3(a)). The distance between these two discs can be adjusted, as well as the center point between the discs which lies in relation to the spinal joint. Each actuator is attached to the discs by two steel Chooks. These hooks can be screwed into and out of the discs to adjust the mounted length of each individual actuator.

In passive and actuated spine morphologies, four actuators were used in the spine, two separated by about $8 \mathrm{~cm}$ above the spine and two below the spine (Fig. 3(a)). The rigid spine is achieved by replacing spinal joint (Fig. 3(b)) with one piece of connecting part (Fig. 3(c)).

\section{E. Electro-Pneumatic System}

We established an electro-pneumatic system to achieve pneumatic control (Fig. 4). Each joint is driven by a pair of antagonistic Mckibben muscles which are actuated by one 3-position proportional value. The valve receives control signals from Arduino through an amplifier. Pressure inside the actuator is captured by a pressure sensor, the angle is measured by a potentiometer, and the state of the robot is sensed by force sensors.

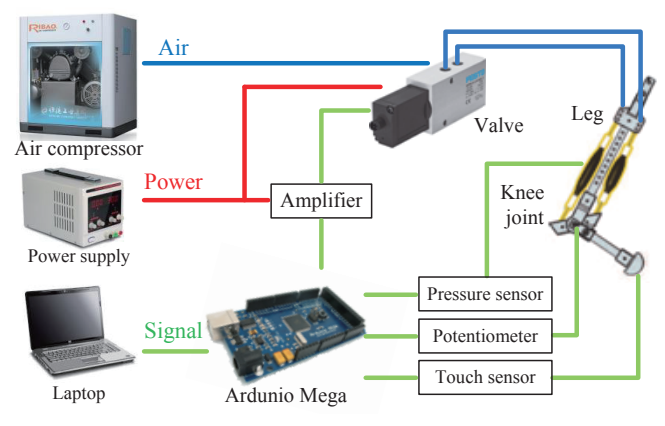

Fig. 4. Overview of the electro-pneumatic system.

\section{F. Controller design}

To focus on the study of the morphology of the spine and reduce the complexity of the controller, a basic sequential step-function control pattern was designed (Fig. 5) for bounding gait. This locomotion pattern, including the coordination between the spine and legs, was designed by observing the movement of a bounding cheetah; the parameters were tuned via trial and error. In Fig. 5, each movement is timed as a percentage of the complete gait cycle period.

\section{EXPERIMENTS}

We conducted experiments to investigate how spine morphology affects locomotion. Three spine morphologies, which are rigid, passive, and actuated spine, were tested in bounding gait. In all of the experiments, four trials for each configuration were conducted and their velocities were recorded and averaged.

\section{A. Experimental setting}

First, we did experiments on rigid spine and selected the best individual at different frequencies. The pressure of hip and knee muscles were kept 4 [bar].

Next, we moved on to the passive case to investigate the effect of stiffness distribution in bounding gait. In this experiments, the parameters were the same as the best individual of rigid case. The lower spine muscles were kept at 4 [bar], making the spinal joint quite stiff but still mildly compliant, behaving like a stiff spring. The pressure of the upper muscle was changed manually from 3 [bar] to 5 [bar] with the increment of 0.5 [bar].

Last, in the actuated spine morphology, we simplified the spinal movements in a way such that when the front legs flex, the spine extends, and when the rear legs flex, the spine flexes, as shown in Fig. 6. This simplification is reasonable, because it is more likely to increase the forward flexion of the limbs and advances the limbs more rapidly, as suggested by biological findings [3]. Therefore, two parameters which are phase delay between the shoulders flexor and the spine extensor ( $\left.\Psi_{\text {ShoulderFlex,SpineExte }}\right)$, and phase delay between the hips flexor and the spine flexor $\left(\Psi_{\text {HipFlex,SpineFlex }}\right)$ are employed to control the spinal movements and coordinate legs movements. Here, positive delay means that the spine is 


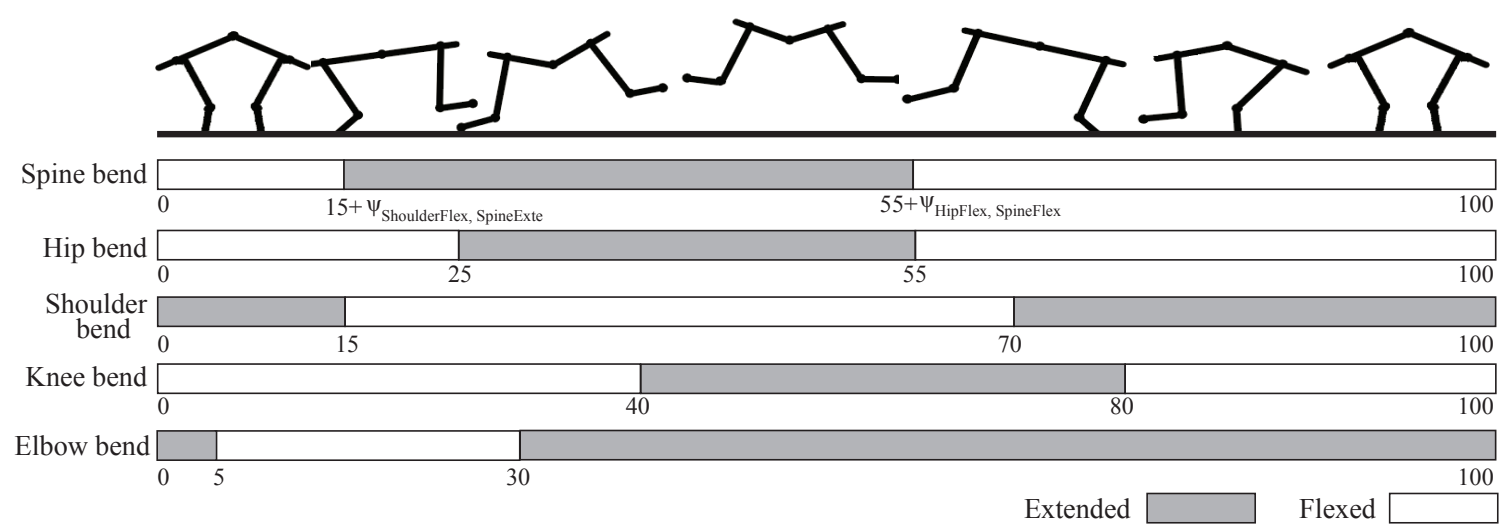

Fig. 5. Proposed value operation scheme for bounding in actuated spine case. The legs actuation is the same for rigid and passive cases.

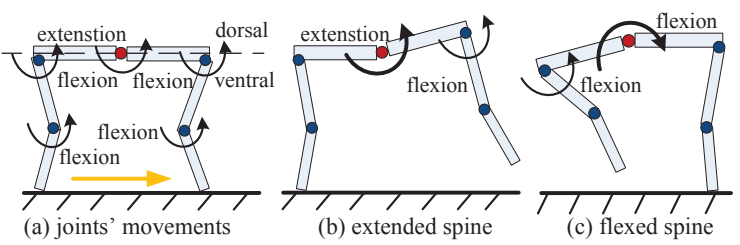

Fig. 6. (a) Description of robot posture. (b) Coordination between extended spine and front legs. (c) Coordination between flexed spine and rear legs. Spinal joint, and legs joints are represented by red and blue dots, respectively. The orange arrow indicates the bounding direction.

actuated later than the corresponding limbs, while a negative value means that the spine is actuated earlier. Except these two new parameters, we chose the rest parameters from the best passive individual and froze them in the actuated case.

\section{B. Results}

1) Rigid and Passive spine: Figure 7 shows the overall performance of Renny when the cycle period $(t)$ and upper muscle stiffness $\left(P_{u p}\right)$ change. We observed that there exists a region where Renny is more likely to move relatively faster. This region expands from $t=0.7$ [s] to $t=0.9$ [s]. Especially when $t=0.82$ [s], the robot runs much faster than other frequencies in most cases. Within this region, more specifically, we found that the overall upper area has higher value than the lower one (Fig. 7). This result suggests that if Renny's upper muscles' pressure is higher than the lower one (4 [bar]), then Renny's speed is even faster. In other words, relatively higher stiffness in the upside of the spine is beneficial to locomotion, which is in agreement with some biological studies [15].

Furthermore, we noticed that the best passive case ( $t=$ 0.82 [s] and $P_{u p}=4.5$ [bar]) is able to reach $98.8 \mathrm{~cm} / \mathrm{s}$, which is faster than the best rigid case whose speed is 83.3 $\mathrm{cm} / \mathrm{s}$. This might be explained by the soft and compliant characteristics of PAMs. One of similarities between PAMs and biological muscles is compliance, which contributes to locomotion by working like a spring transferring energy

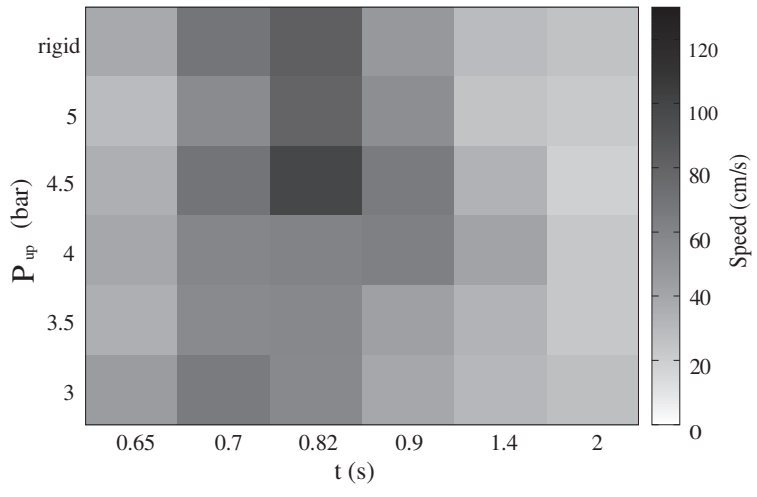

Fig. 7. 2D mapped average velocity for the passive spine experiments with different pressures for the upper spine muscles $P_{u p}$ and different gait cycle $t$, while the pressure for the lower spine muscles is kept 4 [bar].

between front body and rear body in bounding gait [3].

Next, we chose the best individual $\left(t=0.82[\mathrm{~s}], P_{u p}=4.5\right.$ [bar]) from passive cases, and analyzed its behavior. Figure 8 shows that the robot always exhibits a flexed spine, because of the lower stiffness in the belly. We also observed that one bounding cycle can be categorized into four phases: double stance, back leg stance, double flight, and front leg stance.

i) Double stance This phase can be used as an initial phase for the robot to start bounding because of its stability. The phase features retraction of all of the legs. Additionally, the spine is slightly flexed by this posture. The transition to the back leg stance is triggered by extending hip joints and flexing shoulder joints. This causes the robot's upper torso and fore legs to lift off the ground.

ii) Back leg stance This phase starts with the fore legs lifting off the ground during the double stance phase. It transits to double flight phase by further extending hip joints and flexing shoulder joints.

iii) Double flight The ground reaction force provides enough force to lift up the back legs and leads to this double flight phase in which hip joints are fully extended 


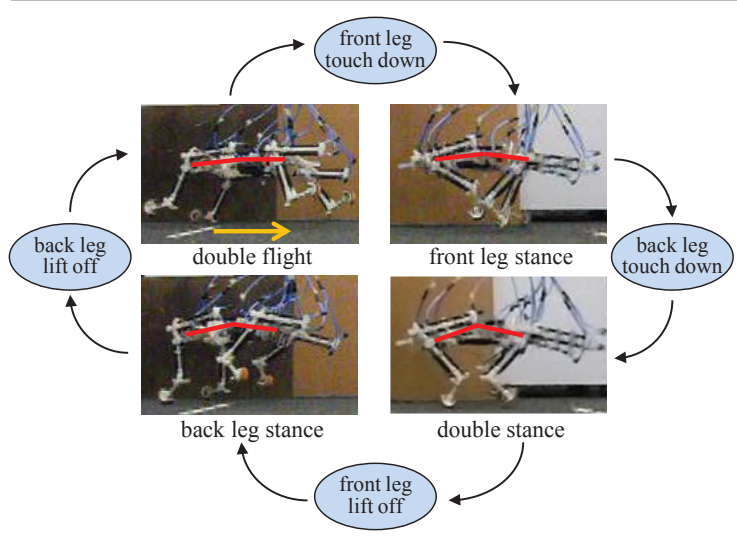

Fig. 8. Bounding phases and events in passive spine morphology of Renny. The orange straight arrow indicates the moving direction.

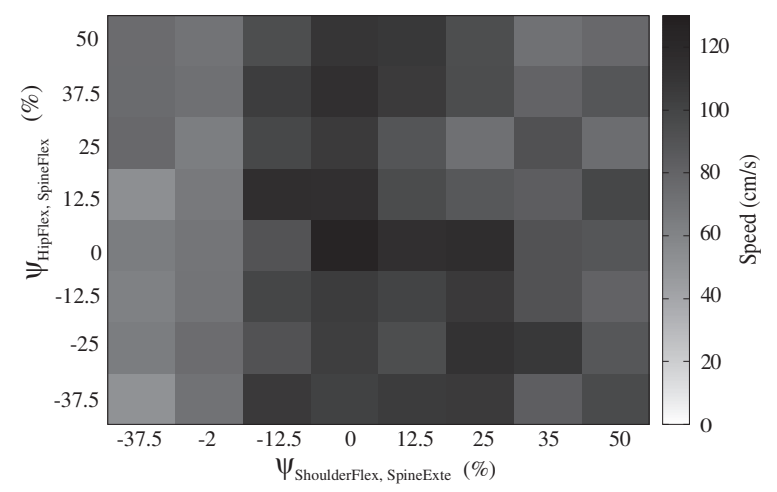

Fig. 9. 2D mapped average velocity for the actuated spine experiments with different phase delays $\Psi_{\text {ShoulderFlex,SpineExte }}$ and $\Psi_{\text {HipFlex,SpineFlex }}$.

are shoulder joints are fully flexed.

iv) Front leg stance When the fore legs touch on the ground, they propel the body forward by extending the shoulder joints. The hip and knee joints are swung forward to increase the stride length.

2) Actuated spine: In the following experiments, we took parameters from the best passive individual $(t=0.82[\mathrm{~s}]$, $\left.P_{u p}=4.5[\mathrm{bar}]\right)$ and copied them to the robot with an actuated spine. In addition, two spinal parameters which are phase delay between spine extensor and front legs flexor $\left(\Psi_{\text {ShoulderFlex,SpineExte }}\right)$ and phase delay between the spine flexor and rear legs flexor $\left(\Psi_{\text {HipFlex,SpineFlex }}\right)$ are explored to study how to control and coordinate the spinal movements.

Figure 9 shows the results of the coordination between the spine and legs on the bounding gait. We observed that it is more likely for the robot to reach faster speed when $\Psi_{\text {ShoulderFlex,SpineExte }}$ is greater than $-12.5 \%$ and less than $25 \%$. It suggests that it exists strong coupling between the spine extension and the front legs flexion, and the resultant timing is better to be as short as possible.

More interestingly, the best individual is found when both $\Psi_{\text {HipFlex,SpineFlex }}$ and $\Psi_{\text {ShoulderFlex,SpineExte }}$ equal zero

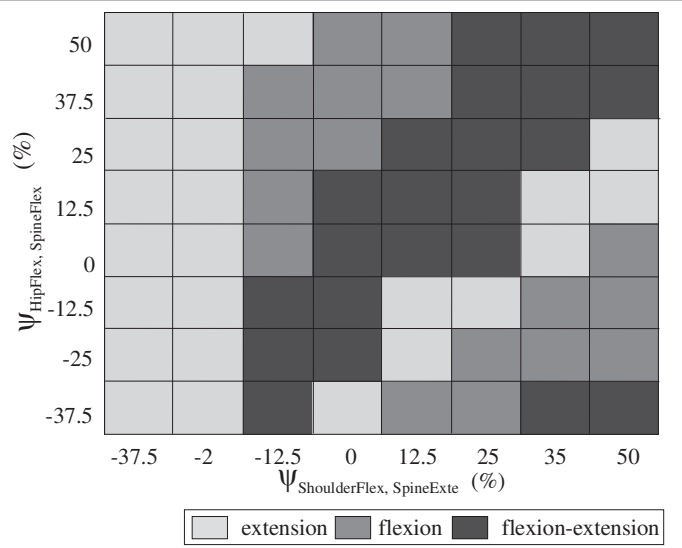

Fig. 10. Three types of spinal movements exhibited in Renny with an actuated spine.

(Fig. 9). This reveals that the synchronization between the spine and legs movements is crucial to increase the speed. Figure 11 shows the performance of this fastest individual and its four phases. The main feature of such morphology is that it can control the spinal movements and coordinate legs. In the double flight phase, the spine is extended to maximize stride length. In the front leg stance phase, the spine flexion benefits to reposition the rear legs, making them quickly reach the double stance phase.

Over all the experiments, we observed three typical spinal movements and categorized them into extended-oscillation, flexed-oscillation, and full-oscillation type (Fig. 10). All of them have the possibilities to reach high speed.

The extended-oscillation type is defined if the spine is always extended more and rarely flexed. As a result, the spinal movements oscillate between marked and nearly no extension. This type increases ground clearance of front legs and stride length of the robot.

The flexed-oscillation type emerges when the spinal movements oscillate between pronounced flexion and nearly no flexion. This spinal movements benefit to retract the rear legs, thus advancing rear legs more rapidly.

The full-oscillation type is expected to outperform the other two types, because it integrates two functions generated by spine flexion and extension. However, it cannot always run faster. This is caused by insufficient ground friction in back leg stance where the back legs are going to lift off and the spine is going to extend. In other words, the interaction between the feet and the ground is not able to produce such high ground friction needed to counteract the torque generated by the rear legs and spinal movements. As a consequence, rear legs feet slide back, thus resulting in slow speed. In nature, cheetahs solve this problem by developing nonretractable claws to enhance grip [16], therefore providing sufficient friction for high bounding gait speeds.

3) Comparisons among these three morphologies: To fairly compare the effect of rigid, passive, and actuated spine on locomotion, we chose the best individuals from these three 


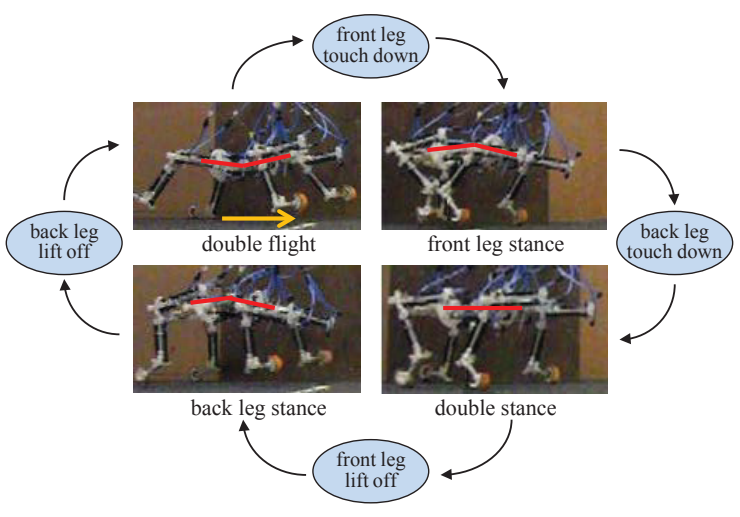

Fig. 11. Bounding phases and events in actuated spine morphology of Renny. The orange straight arrow indicates the moving direction.

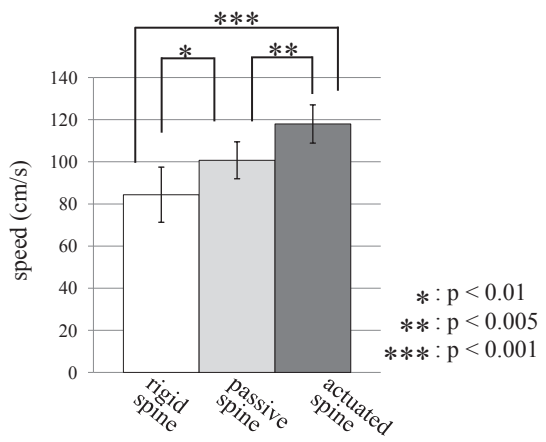

Fig. 12. Comparison among the best individuals from rigid spine, passive spine, and actuated spine. The error bars show the standard deviation. The averaged velocity for rigid, passive, and actuated spine are $84.4 \pm 13.8$, $100.7 \pm 9.2$, and $118 \pm 9.6$, respectively. Asterisks indicate significant differences. $*: p<0.01, * *: p<0.005$, and $* * *: p<0.001$.

morphologies and record the speed and standard derivation over ten trials. To assess how different among these three individuals, one way analysis of variance (ANOVA) has been applied and Tukey-Kramer multiple comparison test has also been employed when results of ANOVA showed significant difference $(p<0.001)$. The overall results are shown in Fig. 12. We observed that there exists significant difference between each pair out of these three morphologies. Robot with actuated spine is the fastest one, largely due to the additional spine actuation which properly extends and flexes the spine to enhance locomotion. Robot with suitable distribution of stiffness runs faster than the rigid case. It might be accounted for by its compliant body, which works like a nonlinear spring to transfer and store energy.

\section{CONCLUSION AND DISCUSSIONS}

In this paper, a pneumatically-driven quadruped robot has been developed to investigate the effect of spine morphology on the bounding gait. Specifically, the actuation type, the stiffness distribution, and the coordination between the spine and the legs were studied. The results showed that a passive spine where dorsal stiffness is higher than ventral stiffness is able to run faster than a rigid case, because of the compliance of the spine, which might play a role as a nonlinear spring to enhance locomotion by transferring and storing energy. Further studies about how to quantify the energy transfer by information theory will be investigated in the future. Generally speaking, a robot with an actuated spine can run faster, due to its additional spine actuation, which generates more power to increase the swing of the limbs and advance the limbs more rapidly, thus contributing to the locomotion. These characteristics might provide design principles for other robots with an actuated spine to enhance locomotion.

In the future, we will improve the hardware by mounting all of the valves into the robot to make it self-contained as much as possible. Furthermore, more complex spine morphologies (more vertebrae and muscles), and movements (twisting, lateral bending, or the combination) will be studied and implemented to this robot, to better understand the role and the effect of the spine on quadruped locomotion.

\section{REFERENCES}

[1] R. M. Alexander, Principles of Animal Locomotion. Princeton University Press, 2002.

[2] N. Schilling and D. Carrier, "Function of the epaxial muscles in walking, trotting and galloping dogs: implications for the evolution of epaxial muscle function in tetrapods," J Exp Biol, pp. 1490-1502, 2010.

[3] M. Hildebrand, "Motions of the running cheetah and horse," Journal of Mammalogy, vol. 40, no. 4, pp. 481-495, 1959.

[4] M. A. Lewis and G. A. Bekey, "Gait adaptation in a quadruped robot," Autonomous Robots, vol. 12, pp. 301-312, 2002.

[5] K. F. Leeser, "Locomtotion experiments on a planar quadruped robot with articulated back spine," Master's thesis, Massachusetts Institute of Technology, 1996.

[6] U. Culha and U. Saranli, "Quadrupedal bounding with an actuated spinal joint," in 2011 IEEE International Conference on Robotics and Automation (ICRA), May 2011, pp. 1392-1397.

[7] R. Pfeifer and J. Bongard, How the Body Shapes the Way We Think: A New View of Intelligence. The MIT Press, 2006.

[8] B. Boszczyk, A. Boszczyk, and P. Reinhard, "Comparative and functional anatomy of the mammalian lumbar spine," The Anatomical Record, vol. 264, no. 2, pp. 157-168, 2001.

[9] Y. Sakai, T. Sato, D. Owaki, and A. Ishiguro, "Realization of stable quadruped gait transition by changing body stiffness," in Proceedings of the 2007 JSME Conference on Robotics and Mechatronics, may 2007, pp. 2A1-2A10.

[10] K. Tsujita and K. Miki, "A study on trunk stiffness and gait stability in quadrupedal locomotion using musculoskeletal robot," in 2011 15th International Conference on Advanced Robotics, 2011, pp. 316-321.

[11] T. Takuma, M. Ikeda, and T. Masuda, "Facilitating multi-modal locomotion in a quadruped robot utilizing passive oscillation of the spine structure," in 2010 IEEE/RSJ International Conference on Intelligent Robots and Systems (IROS), 2010, pp. 4940-4945.

[12] G. K. Klute, J. M. Czerniecki, and B. Hannaford, "Mckibben artificial muscles: Pneumatic actuators with biomechanical intelligence," in In Proceedings on IEEE/ASME 1999 International Conference on Advanced Intelligent Mechatronics, 1999, pp. 221-226.

[13] F. Daerden and D. Lefeber, "Pneumatic artificial muscles: actuators for robotics and automation," European journal of Mechanical and Environmental Engineering, vol. 47, pp. 10-21, 2000.

[14] C. Chou and B. Hannaford, "Measurement and modeling of mckibben pneumatic artificial muscles," Robotics and Automation, IEEE Transactions on, vol. 12, no. 1, pp. 90-102, 1996.

[15] J. Long, "Locomotor design of dolphin vertebral columns: bending mechanics and morphology of Delphinus delphis," Journal of Experimental Biology, vol. 200, no. 1, pp. 65-81, 1997.

[16] A. M. Wilson, J. C. Lowe, K. Roskilly, P. E. Hudson, K. A. Golabek, and J. W. McNutt, "Locomotion dynamics of hunting in wild cheetahs," Nature, vol. 498, pp. 185-189, 2013. 

Appendix F

Curriculum Vitae 


\title{
Curriculum Vitae
}

\author{
Last name: Zhao \\ First name: Qian \\ Date of birth: December 11, 1981
}

Nationality: China

\section{Education}

11/2008-06/2014 Ph.D. candidate and research assistant at the Artificial Intelligence Laboratory, Department of Informatics, University of Zurich, Switzerland Ph.D. thesis: Spine as an Engine: Effect of Spine Morphology on SpineDriven Quadruped Locomotion

09/2005-04/2008 Master in Mechanical Engineering, Department of Industrial Engineering, Northwestern Polytechnic University, China

09/2000-07/2004 Bachelor in Mechanical Engineering, Department of Mechanic Engineering, Xi' an Technological University, China 